\title{
Edge Even Graceful Labeling of Cylinder Grid Graph
}

\author{
Ahmed A. Elsonbaty ${ }^{1,2}$ and Salama Nagy Daoud 1,3,*(D) \\ 1 Department of Mathematics, Faculty of Science, Taibah University, Al-Madinah 41411, Saudi Arabia; \\ ahmad_elsonbaty@hotmail.com \\ 2 Department of Mathematics, Faculty of Science, Ain Shams University, Cairo 11566, Egypt \\ 3 Department of Mathematics and Compuer Science, Faculty of Science, Menoufia University, \\ Shebin El Kom 32511, Egypt \\ * Correspondence: sdaoud@taibahu.edu.sa
}

Received: 26 March 2019; Accepted: 14 April 2019; Published: 22 April 2019

check for updates

\begin{abstract}
Edge even graceful labeling (e.e.g., 1.) of graphs is a modular technique of edge labeling of graphs, introduced in 2017. An e.e.g., 1. of simple finite undirected graph $G=(V(G), E(G))$ of order $P=\mid(V(G) \mid$ and size $q=|E(G)|$ is a bijection $f: E(G) \rightarrow\{2,4, \ldots, 2 q\}$, such that when each vertex $v \in V(G)$ is assigned the modular sum of the labels (images of $f$ ) of the edges incident to $v$, the resulting vertex labels are distinct $\bmod 2 r$, where $r=\max (p, q)$. In this work, the family of cylinder grid graphs are studied. Explicit formulas of e.e.g., l. for all of the cases of each member of this family have been proven.
\end{abstract}

Keywords: graceful labeling; edge even graceful labeling; cylinder grid graph

\section{Introduction}

The field of graph theory plays an important role in various areas of pure and applied sciences. One of the important areas in graph theory is graph labeling of a graph $G$ which is an assignment of integers either to the vertices or edges or both subject to certain conditions. Graph labeling began nearly 50 years ago. Over these decades, more than 200 methods of labeling techniques were invented, and more than 2500 papers were published. In spite of this huge literature, just few general results were discovered. Nowadays, graph labeling has much attention from different brilliant researchers in graph theory, which has rigorous applications in many disciplines, e.g., communication networks, coding theory, X-ray crystallography, radar, astronomy, circuit design, communication network addressing, database management, and graph decomposition problems. More interesting applications of graph labeling can be found in References [1-11]. A function $f$ is called a graceful labeling of a graph $G$ if $f: V(G) \rightarrow\{0,1,2, \ldots, q\}$ is injective and the induced function $f^{*}: E(G) \rightarrow\{1,2, \ldots, q\}$, defined as $f^{*}(e=u v)=|f(u)-f(v)|$, is bijective. This type of graph labeling was first introduced by Rosa in 1967 [12] as a $\beta$ - valuation, and later, Solomon W. Golomb [13] termed it as graceful labeling. A function $f$ is called an odd graceful labeling of a graph $G$ if $f: V(G) \rightarrow\{0,1,2, \ldots, 2 q-1\}$ is injective and the induced function $f^{*}: E(G) \rightarrow\{1,3, \ldots, 2 q-1\}$, defined as $f^{*}(e=u v)=|f(u)-f(v)|$, is bijective. This type of graph labeling first introduced by Gnanajothi in 1991 [14]. For more results on this type of labeling, see References [15,16]. A function $f$ is called an edge graceful labeling of a graph $G$ if $f: E(G) \rightarrow\{1,2, \ldots, q\}$ is bijective and the induced function $f^{*}: V(G) \rightarrow\{0,1,2, \ldots, p-1\}$, defined as $f^{*}(u)=\sum_{e=u v \in E(G)} f(e)(\bmod p)$, is bijective. This type of graph labeling was first introduced by Lo in 1985 [17]. For more results on this labeling see [18,19]. A function $f$ is called an edge odd graceful labeling of a graph $G$ if $f: E(G) \rightarrow\{1,3, \ldots, 2 q-1\}$ is bijective and the induced function $f^{*}: V(G) \rightarrow\{0,1,2, \ldots, 2 q-1\}$ defined as $f^{*}(u)=\sum_{e=u v \in E(G)} f(e)(\bmod 2 q)$ is injective. This type of 
graph labeling was first introduced by Solairaju and Chithra in 2009 [20]. For more results on this labeling, see References [21-23]. A function $f$ is called an edge even graceful labeling of a graph $G$ if $f: E(G) \rightarrow\{2,4, \ldots, 2 q-2\}$ is bijective and the induced function $f^{*}: V(G) \rightarrow\{0,2,4, \ldots, 2 q-2\}$, defined as $f^{*}(u)=\sum_{e=u v \in E(G)} f(e)(\bmod 2 r)$ where $r=\max \{p, q\}$, is injective. This type of graph labeling was first introduced by Elsonbaty and Daoud in 2017 [24,25]. For a summary of the results on these five types of graceful labels as well as all known labeling techniques, see Reference [26].

\section{Cylinder Grid Graph}

The Cartesian product $G_{1} \times G_{2}$ of two graphs $G_{1}$ and $G_{2}$, is the graph with vertex set $V\left(G_{1}\right) \times V\left(G_{2}\right)$, and any two vertices $\left(u_{1}, v_{1}\right)$ and $\left(u_{2}, v_{2}\right)$ are adjacent in $G_{1} \times G_{2}$ whenever $u_{1}=u_{2}$ and $v_{1} v_{2} \in E\left(G_{2}\right)$ or $v_{1}=v_{2}$ and $u_{1} u_{2} \in E\left(G_{1}\right)$. The cylinder grid graph $C_{m, n}$ is the graph formed from the Cartesian product $P_{m} \times C_{n}$ of the path graph $P_{m}$ and the cycle graph $C_{n}$. That is, the cylinder grid graph consists of $m$ copies of $C_{n}$ represented by circles, and will be numbered from the innermost circle to the outer circle as $C_{n}^{(1)}, C_{n}^{(2)}, C_{n}^{(3)}, \ldots, C_{n}^{(m-1)}, C_{n}^{(m)}$ and we call them simply circles; $n$ copies of $P_{m}$ represented by paths transverse the $m$ circles and will be numbered clockwise as $P_{m}^{(1)}, P_{m}^{(2)}, P_{m}^{(3)}, \ldots, P_{m}^{(n-1)}, P_{m}^{(n)}$ and we call them paths (see Figure 1).

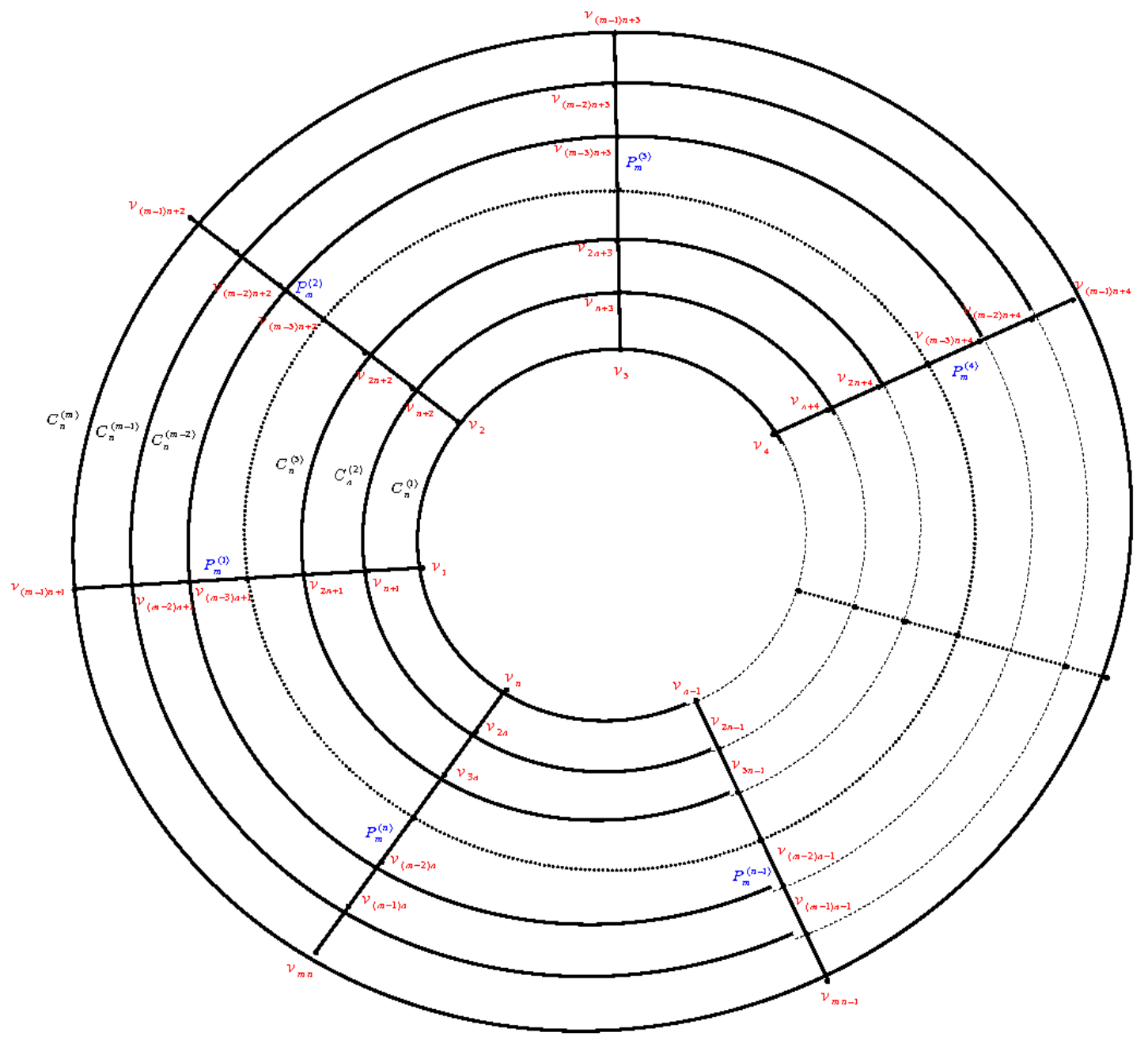

Figure 1. Cylinder grid graph $C_{m, n}$. 
Theorem 1. If $m$ is an even positive integer greater than or equal 2 and $n \geq 2$, then the cylinder grid graph $C_{m, n}$, is an edge even graceful graph.

Proof. Using standard notation $p=\left|V\left(C_{m, n}\right)\right|=m n, q=\left|E\left(C_{m, n}\right)\right|=2 m n-n$ and $r=\max (p, q)=2 m n-n$ and $f: E\left(C_{m, n}\right) \rightarrow\{2,4,6, \ldots, 4 m n-2 n-2\}$. Let the cylinder grid graph $C_{m, n}$ be as in Figure 2.

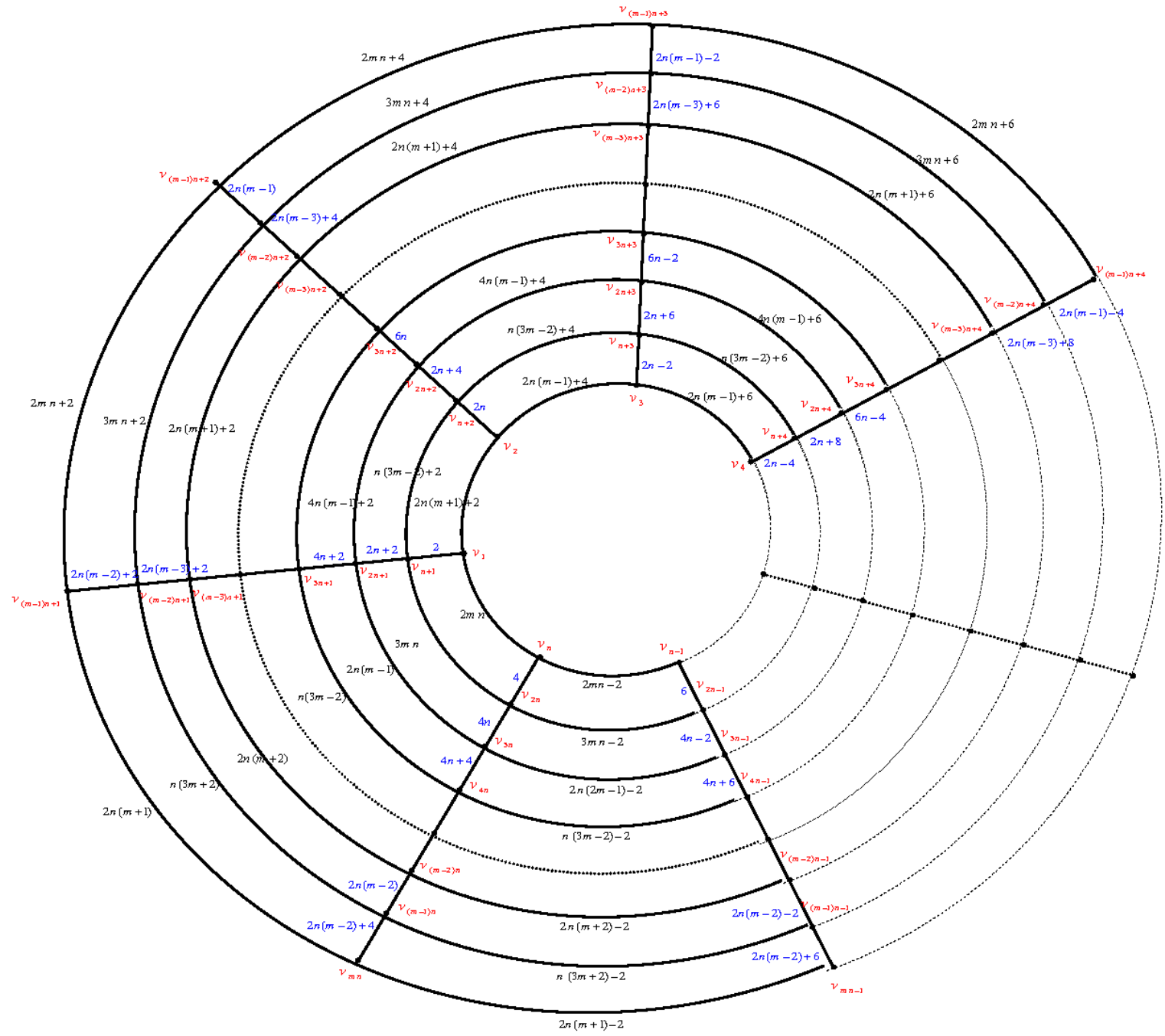

Figure 2. The cylinder grid graph $C_{m, n}, m$ is even and $n \geq 2$.

First, we label the edges of the paths $P_{m}^{(k)}, 1 \leq k \leq n$ beginning with the edges of the path $P_{m}^{(1)}$ as follows: Move anticlockwise to label the edges $v_{1} v_{n+1}, v_{n} v_{2 n}, v_{n-1} v_{2 n-1}, \ldots, v_{3} v_{n+3}, v_{2} v_{n+2}$ by $2,4,6, \ldots, 2 n-2,2 n$, then move clockwise to label the edges $v_{n+1} v_{2 n+1}, v_{n+2} v_{2 n+2}, v_{n+3} v_{2 n+3}, \ldots$, $v_{2 n-1} v_{3 n-1}, v_{2 n} v_{3 n}$ by $2 n+2,2 n+4,2 n+6, \ldots, 4 n-2,4 n$, then move anticlockwise to label the edges $v_{2 n+1} v_{3 n+1}, v_{3 n} v_{4 n}, v_{3 n-1} v_{4 n-1}, \ldots, v_{2 n+3} v_{3 n+3}, v_{2 n+2} v_{3 n+2}$ by $4 n+2,4 n+4,4 n+6, \ldots, 6 n-2,6 n$ and so on. Finally, move anticlockwise to label the edges $v_{(m-2) n+1} v_{(m-1) n+1}, v_{(m-1) n} v_{m n}, v_{(m-1) n-1} v_{m n-1}, \ldots$, $v_{(m-2) n+3} v_{(n-1) m+3}, v_{(m-2) n+2} v_{(m-1) n+2}$ by $2 n(m-1)+2,2 n(m-2)+4,2 n(m-2)+6,2 n(m-2)+$ $8, \ldots, 2 n(m-1)-2,2 n(m-1)$.

Secondly, we label the edges of the circles $C_{n}^{(k)}, 1 \leq k \leq m$ beginning with the edges of the innermost circle $C_{n}^{(1)}$ then the edges of outer circle $C_{n}^{(m)}$, then the edges of the circles $C_{n}^{(m-2)}, C_{n}^{(m-4)}, \ldots, C_{n}^{(2)}$.

Finally, we label the edges of the circles $C_{n}^{(m-1)}, C_{n}^{(m-3)}, \ldots, C_{n}^{(3)}$ as follows: $f\left(v_{i} v_{i+1}\right)=2 n(m-1)+$ $2 i, 1 \leq i \leq n-1, f\left(v_{n} v_{1}\right)=2 m n ; f\left(v_{(m-1) n+i} v_{(m-1) n+i+1}\right)=2 m n+2 i, 1 \leq i \leq n-1, f\left(v_{m n} v_{(m-1) n+1}\right)=$ 
$2 n(m+1) ; f\left(v_{(k-1) n+i} v_{(k-1) n+i+1}\right)=n(3 m-k)+2 i, 1 \leq i \leq n-1, f\left(v_{k n} v_{(k-1) n+1}\right)=n(3 m-k+2), 2 \leq$ $k \leq m-2 ; f\left(v_{(k-1) n+i} v_{(k-1) n+i+1}\right)=n(4 m-k-1)+2 i, 1 \leq i \leq n-1, f\left(v_{k n} v_{(k-1) n+1}\right)=n(4 m-k+1)$, $3 \leq k \leq m-1, k$ is odd.

Thus, the labels of corresponding vertices $\bmod (4 m n-2 n)$ will be: $f^{*}\left(v_{i}\right) \equiv 2 i+2 ; f^{*}\left(v_{n+i}\right) \equiv$ $2 m n+2 n+4 i+2 ; f^{*}\left(v_{2 n+i}\right) \equiv 4 n+4 i+2 ; f^{*}\left(v_{3 n+i}\right) \equiv 2 m n+6 n+4 i+2 ; \ldots ; f^{*}\left(v_{(m-3) n+i}\right) \equiv 4 m n-$ $6 n+4 i+2 ; f^{*}\left(v_{(m-2) n+i}\right) \equiv 2 m n-4 n+4 i+2 ; f^{*}\left(v_{(m-1) n+i}\right) \equiv 2 m n+2 i+2,1 \leq i \leq n$.

Illustration: An e.e.g., 1 , of the cylinder grid graphs $C_{8,11}$ and $C_{8,12}$ are shown in Figure 3.

Theorem 2. If $m=3$ and $n$ is an odd positive integer greater than 3 , then the cylinder grid graph $C_{3, n}$, is an edge even graceful graph.

Proof. Using standard notation $p=\left|V\left(C_{3, n}\right)\right|=3 n, q=\left|E\left(C_{3, n}\right)\right|=5 n, r=\max (p, q)=5 n$, and $f: E\left(C_{3, n}\right) \rightarrow\{2,4,6, \ldots, 10 n-2\}$. There are three cases:

Case (1): If $n \equiv 1 \bmod 6$, let the cylinder grid graph $C_{3, n}$ be as in Figure 4 .

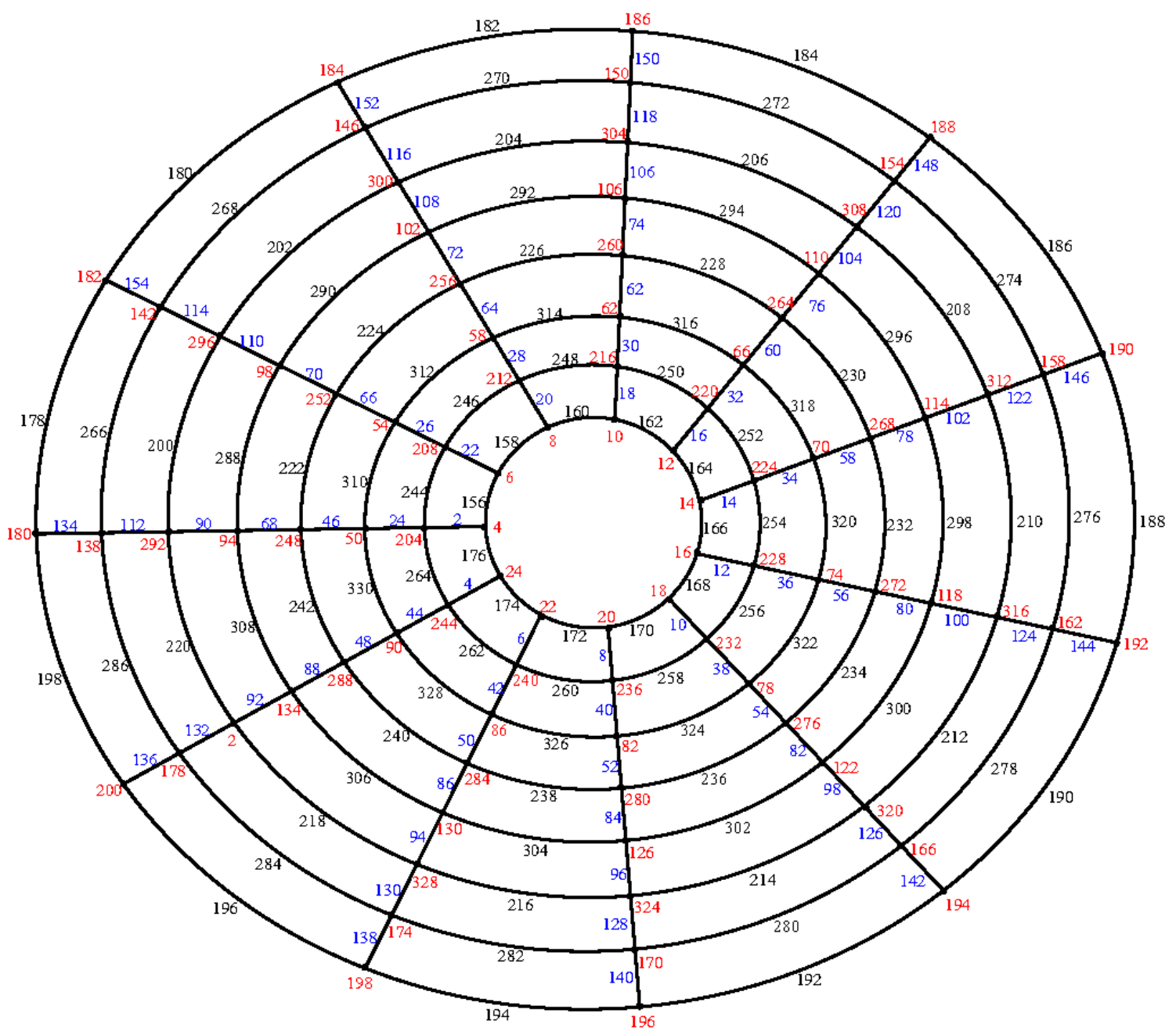

(a) $C_{8,11}$

Figure 3. Cont. 


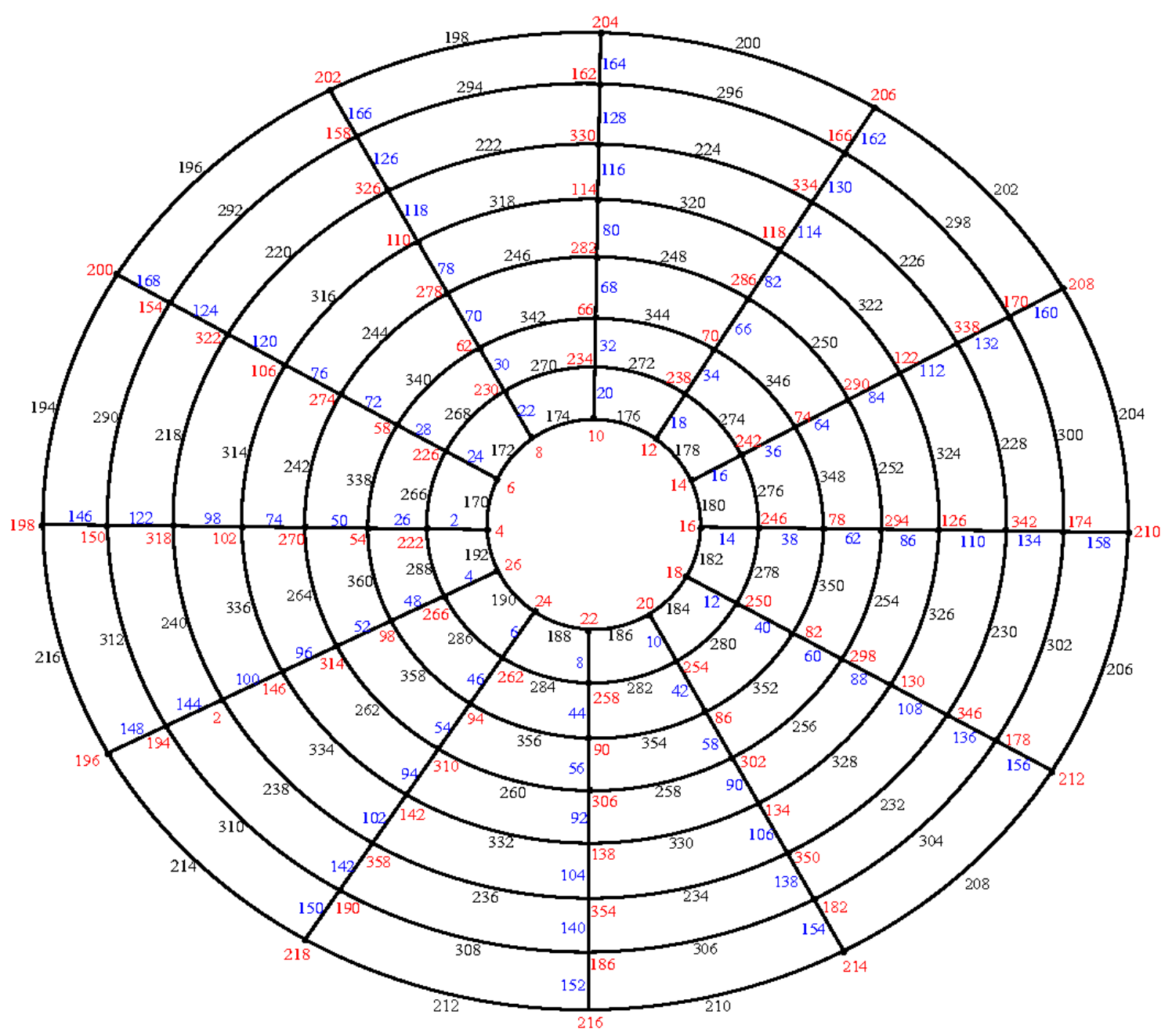

(b) $C_{8,12}$

Figure 3. An edge even graceful labeling (e.e.g., 1.) of the cylinder grid graphs $C_{8,11}$ and $C_{8,12}$.

First, we label the edges of the paths $P_{3}^{(k)}, 1 \leq k \leq n$ beginning with the edges of the path $P_{3}^{(1)}$ as follows: Move clockwise to label the edges $f\left(v_{1} v_{n+1}\right)=2, f\left(v_{2} v_{n+2}\right)=6, f\left(v_{i} v_{n+i}\right)=$ $2 i+2,3 \leq i \leq n$. Then, move anticlockwise to label the edges $f\left(v_{n+1} v_{2 n+1}\right)=2 n+4, f\left(v_{2 n} v_{3 n}\right)=2 n+$ $6, f\left(v_{2 n-1} v_{3 n-1}\right)=2 n+8, f\left(v_{2 n-2} v_{3 n-2}\right)=2 n+10, \ldots, f\left(v_{n+3} v_{2 n+3}\right)=4 n, f\left(v_{n+2} v_{2 n+2}\right)=4 n+2$.

Secondly, we label the edges of the circles $C_{n}^{(k)}, 1 \leq k \leq 3$ beginning with the edges of the innermost circle $C_{n}^{(1)}$, then the edges of outer circle $C_{n}^{(3)}$, and then the edges of the circle $C_{n}^{(2)}$. Label the edges of the circle $C_{n}^{(1)}$ as follows: $f\left(v_{1} v_{2}\right)=4 n+4, f\left(v_{2} v_{3}\right)=4 n+6, \ldots, f\left(v_{\frac{n-1}{3}} v_{\frac{n+2}{3}}\right)=\frac{14 n+10}{3}, f\left(v_{\frac{n+2}{3}} v_{\frac{n+5}{3}}\right)=$ $\frac{14 n+4}{3}, f\left(v_{\frac{n+5}{3}} v_{\frac{n+8}{3}}\right)=\frac{14 n+16}{3}, f\left(v_{\frac{n+8}{3}} v_{\frac{n+11}{3}}\right)=\frac{14 n+22}{3}, f\left(v_{\frac{n+11}{3}} v_{\frac{n+14}{3}}\right)=\frac{14 n+34}{3}, f\left(v_{\frac{n+14}{3}} v_{\frac{n+17}{3}}\right)=$ $\frac{14 n+28}{3}, f\left(v_{\frac{n+17}{3}} v_{\frac{n+20}{3}}\right)=\frac{14 n+40}{3}, f\left(v_{\frac{n+20}{3}} v_{\frac{n+23}{3}}\right)=\frac{14 n+46}{3}, f\left(v_{\frac{n+23}{3}} v_{\frac{n+26}{3}}\right)=\frac{14 n+58}{3}, f\left(v_{\frac{n+26}{3}} v_{\frac{n+29}{3}}\right)=$ $\frac{14 n+52}{3}, f\left(v_{\frac{n+29}{3}}^{3} v_{\frac{n+32}{3}}\right)=\frac{14 n+64}{3}, f\left(v_{\frac{n+32}{3}} v_{\frac{n+35}{3}}^{3}\right)=\frac{14 n+70}{3}, f\left(v_{\frac{n+35}{3}}^{3} v_{\frac{n+38}{3}}\right)=\frac{14 n+82}{3}, f\left(v_{\frac{n+38}{3}} v_{\frac{n+41}{3}}\right)=$ $\frac{14 n+76}{3}, f\left(v_{\frac{n+41}{3}} v_{\frac{n+44}{3}}\right)=\frac{14 n+88}{3}, f\left(v_{\frac{n+44}{3}} v_{\frac{n+47}{3}}\right)=\frac{14 n+94}{3}, \ldots, f\left(v_{n-13} v_{n-12}\right)=6 n-22, f\left(v_{n-12} v_{n-11}\right)=$ $6 n-24, f\left(v_{n-11} v_{n-10}\right)=6 n-20, f\left(v_{n-10} v_{n-9}\right)=6 n-18, f\left(v_{n-9} v_{n-8}\right)=6 n-14, f\left(v_{n-8} v_{n-7}\right)=$ $6 n-16, f\left(v_{n-7} v_{n-6}\right)=6 n-12, f\left(v_{n-6} v_{n-5}\right)=6 n-10, f\left(v_{n-5} v_{n-4}\right)=6 n-6, f\left(v_{n-4} v_{n-3}\right)=6 n-$ $8, f\left(v_{n-3} v_{n-2}\right)=6 n-4, f\left(v_{n-2} v_{n-1}\right)=6 n-2, f\left(v_{n-1} v_{n}\right)=6 n+2, f\left(v_{n} v_{1}\right)=6 n$. 


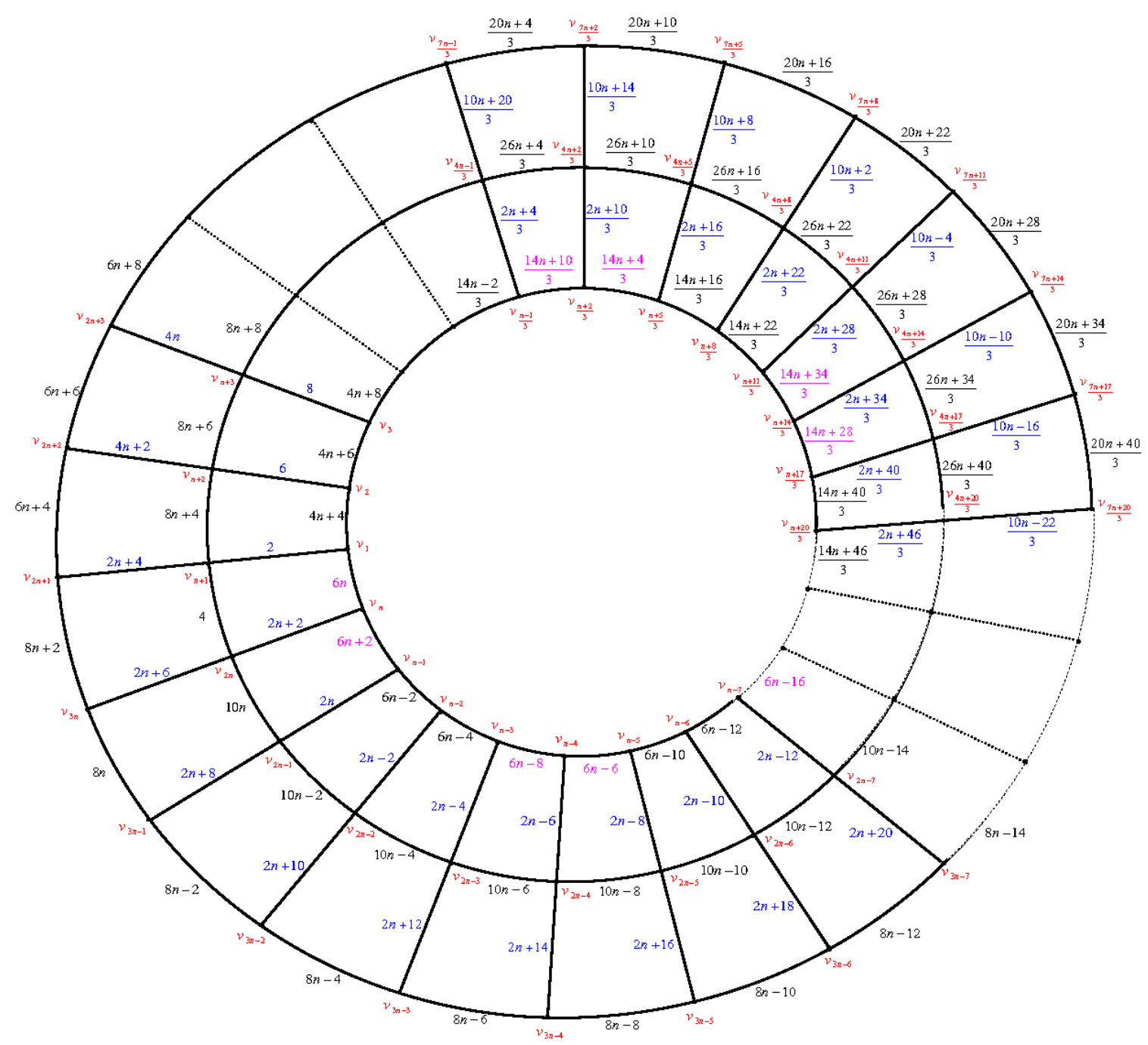

Figure 4. The cylinder grid graph $C_{3, n}, n \equiv 1 \bmod 6$.

Label the edges of the circle $C_{n}^{(2)}$ as follows: $f\left(v_{n+i} v_{n+i+1}\right)=8 n+2 i+2,1 \leq i \leq n-1, f\left(v_{2 n} v_{n+1}\right)=4$. Label the edges of the circle $C_{n}^{(3)}$ as follows: $f\left(v_{2 n+i} v_{2 n+i+1}\right)=6 n+2 i+2,1 \leq i \leq n$.

The labels of corresponding of vertices mod10n are as follows:

The labels of vertices of the circle $C_{n}^{(1)}$ are as follows: $f^{*}\left(v_{1}\right) \equiv 6, f^{*}\left(v_{2}\right) \equiv 8 n+16, f^{*}\left(v_{3}\right) \equiv$ $8 n+22, \ldots, f^{*}\left(v_{\frac{n-1}{3}}\right) \equiv 4, f^{*}\left(v_{\frac{n+2}{3}}\right) \equiv 8, f^{*}\left(v_{\frac{n+5}{3}}\right) \equiv 12, f^{*}\left(v_{\frac{n+8}{3}}\right) \equiv 20, f^{*}\left(v_{\frac{n+11}{3}}\right) \equiv 28, f^{*}\left(v_{\frac{n+14}{3}}\right) \equiv$ $32, f^{*}\left(v_{\frac{n+17}{3}}\right) \equiv 36, f^{*}\left(v_{\frac{n+20}{3}}\right) \equiv 44, f^{*}\left(v_{\frac{n+23}{3}}\right) \equiv 52, f^{*}\left(v_{\frac{n+26}{3}}\right)^{3} \equiv 56, f^{*}\left(v_{\frac{n+29}{3}}\right)^{3} \equiv 60, f^{*}\left(v_{\frac{n+32}{3}}\right)^{3} \equiv 68$, $f^{*}\left(v_{\frac{n+35}{3}}\right) \equiv 76, f^{*}\left(v_{\frac{n+38}{3}}\right) \equiv 80, f^{*}\left(v_{\frac{n+41}{3}}\right) \equiv 84, f^{*}\left(v_{\frac{n+44}{3}}\right) \equiv 92, f^{*}\left(v_{\frac{n+47}{3}}\right) \equiv 100, \ldots, f^{*}\left(v_{n-12}\right) \equiv 4 n-$ $68, f^{*}\left(v_{n-11}\right) \equiv 4 n-64, f^{*}\left(v_{n-10}\right) \equiv 4 n-56, f^{*}\left(v_{n-9}\right) \equiv 4 n-48, f^{*}\left(v_{n-8}\right) \equiv 4 n-44, f^{*}\left(v_{n-7}\right) \equiv 4 n-$ $40, f^{*}\left(v_{n-6}\right) \equiv 4 n-32, f^{*}\left(v_{n-5}\right) \equiv 4 n-24, f^{*}\left(v_{n-4}\right) \equiv 4 n-20, f^{*}\left(v_{n-3}\right) \equiv 4 n-16, f^{*}\left(v_{n-2}\right) \equiv 4 n-$ $8, f^{*}\left(v_{n-1}\right) \equiv 4 n, f^{*}\left(v_{n}\right) \equiv 4 n+4$.

The labels of vertices of the circle $C_{n}^{(2)}$ are $f^{*}\left(v_{i+1}\right)=4 i+10,1 \leq i \leq n-1, f^{*}\left(v_{2 n}\right)=4 n+12$.

The labels of vertices of the circle $C_{n}^{(3)}$ are $f^{*}\left(v_{2 i+1}\right)=6 n+2 i+8,1 \leq i \leq n$.

Case (2): If $n \equiv 3 \bmod 6$, let the cylinder grid graph $C_{3, n}$ be as in Figure 5 .

First, we label the edges of the paths $P_{3}^{(k)}, 1 \leq k \leq n$ beginning with the edges of the path $P_{3}^{(1)}$ as the same in case (1).

Secondly, we label the edges of the circles $C_{n}^{(k)}, 1 \leq k \leq 3$ beginning with the edges of the innermost circle $C_{n}^{(1)}$, then the edges of outer circle $C_{n}^{(3)}$, and then the edges of the circle $C_{n}^{(2)}$. 


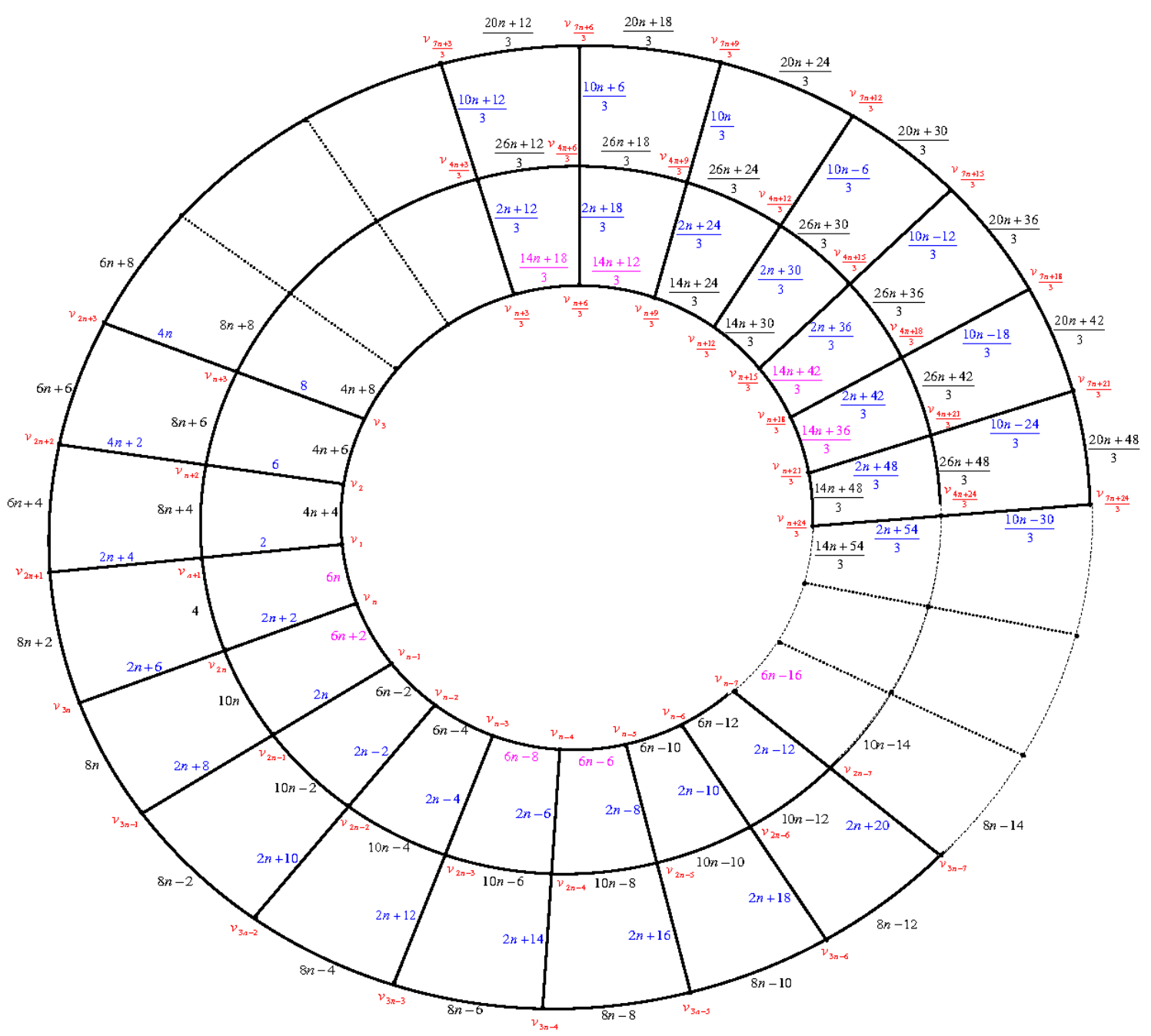

Figure 5. The cylinder grid graph $C_{3, n}, n \equiv 3 \bmod 6$.

Label the edges of the circle $C_{n}^{(1)}$ as follows: $f\left(v_{1} v_{2}\right)=4 n+4, f\left(v_{2} v_{3}\right)=4 n+6, \ldots, f\left(v_{\frac{n+3}{3}} v_{\frac{n+6}{3}}\right)=$ $\frac{14 n+18}{3}, f\left(v_{\frac{n+6}{3}} v_{\frac{n+9}{3}}\right)=\frac{14 n+12}{3}, f\left(v_{\frac{n+9}{3}} v_{\frac{n+12}{3}}\right)=\frac{14 n+24}{3}, f\left(v_{\frac{n+12}{3}} v_{\frac{n+15}{3}}\right)=\frac{14 n+30}{3}, f\left(v_{\frac{n+15}{3}} v_{\frac{n+18}{3}}\right)=$ $\frac{14 n+42}{3}, f\left(v_{\frac{n+18}{3}}^{3} v_{\frac{n+21}{3}}\right)=\frac{14 n+36}{3}, f\left(v_{\frac{n+21}{3}} v_{\frac{n+24}{3}}\right)=\frac{14 n+48}{3}, f\left(v_{\frac{n+24}{3}} v_{\frac{n+27}{3}}\right)=\frac{14 n+54}{3}, f\left(v_{\frac{n+27}{3}} v_{\frac{n+30}{3}}\right)=$ $\frac{14 n+66}{3}, f\left(v_{\frac{n+30}{3}}^{3} v_{\frac{n+33}{3}}^{3}\right)=\frac{14 n+60}{3}, f\left(v_{\frac{n+33}{3}} v_{\frac{n+36}{3}}^{3}\right)=\frac{14 n+72}{3}, f\left(v_{\frac{n+36}{3}} v_{\frac{n+39}{3}}\right)=\frac{14 n+78}{3}, f\left(v_{\frac{n+39}{3}} v_{\frac{n+42}{3}}^{3}\right)=$ $\frac{14 n+90}{3}, f\left(v_{\frac{n+42}{3}} v_{\frac{n+45}{3}}\right)=\frac{14 n+84}{3}, f\left(v_{\frac{n+45}{3}} v_{\frac{n+48}{3}}\right)=\frac{14 n+96}{3}, f\left(v_{\frac{n+48}{3}} v_{\frac{n+51}{3}}\right)=\frac{14 n+102}{3}, \ldots, f\left(v_{n-13} v_{n-12}\right)=$ $6 n-22, f\left(v_{n-12} v_{n-11}\right)=6 n-24, f\left(v_{n-11} v_{n-10}\right) f\left(v_{n-9} v_{n-8}\right)=6 n-14, f\left(v_{n-8} v_{n-7}\right)=6 n-16$, $f\left(v_{n-7} v_{n-6}\right)=6 n-12, f\left(v_{n-6} v_{n-5}\right)=6 n-10, f\left(v_{n-5} v_{n-4}\right)=6 n-6, f\left(v_{n-4} v_{n-3}\right)=6 n-$ $8, f\left(v_{n-3} v_{n-2}\right)=6 n-4, f\left(v_{n-2} v_{n-1}\right)=6 n-2, f\left(v_{n-1} v_{n}\right)=6 n+2, f\left(v_{n} v_{1}\right)=6 n$.

The labels of corresponding vertices mod10 $n$ are as follows: The label of vertices of the circle $C_{n}^{(1)}$ are $f^{*}\left(v_{1}\right) \equiv 6, f^{*}\left(v_{2}\right) \equiv 8 n+16, f^{*}\left(v_{3}\right) \equiv 8 n+22, \ldots, f^{*}\left(v_{\frac{n}{3}-1}\right) \equiv 10 n-2, f^{*}\left(v_{\frac{n}{3}}\right) \equiv 4, f^{*}\left(v_{\frac{n}{3}+1}\right) \equiv$ $12, f^{*}\left(v_{\frac{n}{3}+2}\right) \equiv 16, f^{*}\left(v_{\frac{n}{3}+3}\right) \equiv 20, f^{*}\left(v_{\frac{n}{3}+4}\right) \equiv 28, f^{*}\left(v_{\frac{n}{3}+5}\right) \equiv 36, f^{*}\left(v_{\frac{n}{3}+6}\right) \equiv 40, f^{*}\left(v_{\frac{n}{3}+7}\right) \equiv$ $44, f^{*}\left(v_{\frac{n}{3}+8}\right) \equiv 52, f^{*}\left(v_{\frac{n}{3}+9}\right) \equiv 60, f^{*}\left(v_{\frac{n}{3}+10}\right) \equiv 64, f^{*}\left(v_{\frac{n}{3}+11}\right) \equiv 68, f^{*}\left(v_{\frac{n}{3}+12}\right) \equiv 76, f^{*}\left(v_{\frac{n}{3}}+13\right) \equiv$ $84, f^{*}\left(v_{\frac{n}{3}+14}\right) \equiv 88, f^{*}\left(v_{\frac{n}{3}+15}\right) \equiv 92, f^{*}\left(v_{\frac{n}{3}+16}\right) \equiv 100, \ldots, f^{*}\left(v_{n-12}\right) \equiv 4 n-68, f^{*}\left(v_{n-11}\right) \equiv 4 n-64$, $f^{*}\left(v_{n-10}\right) \equiv 4 n-56, f^{*}\left(v_{n-9}\right) \equiv 4 n-48, f^{*}\left(v_{n-8}\right) \equiv 4 n-44, f^{*}\left(v_{n-7}\right) \equiv 4 n-40, f^{*}\left(v_{n-6}\right) \equiv 4 n-$ $32, f^{*}\left(v_{n-5}\right) \equiv 4 n-24, f^{*}\left(v_{n-4}\right) \equiv 4 n-20, f^{*}\left(v_{n-3}\right) \equiv 4 n-16, f^{*}\left(v_{n-2}\right) \equiv 4 n-8, f^{*}\left(v_{n-1}\right) \equiv 4 n, f^{*}\left(v_{n}\right) \equiv$ $4 n+4$.

The labels of vertices of the circles $C_{n}^{(2)}$ and $C_{n}^{(3)}$ are the same as in case (1).

Case (3): If $n \equiv 5 \bmod 6$, let the cylinder grid graph $C_{3, n}$ be as in Figure 6 . 


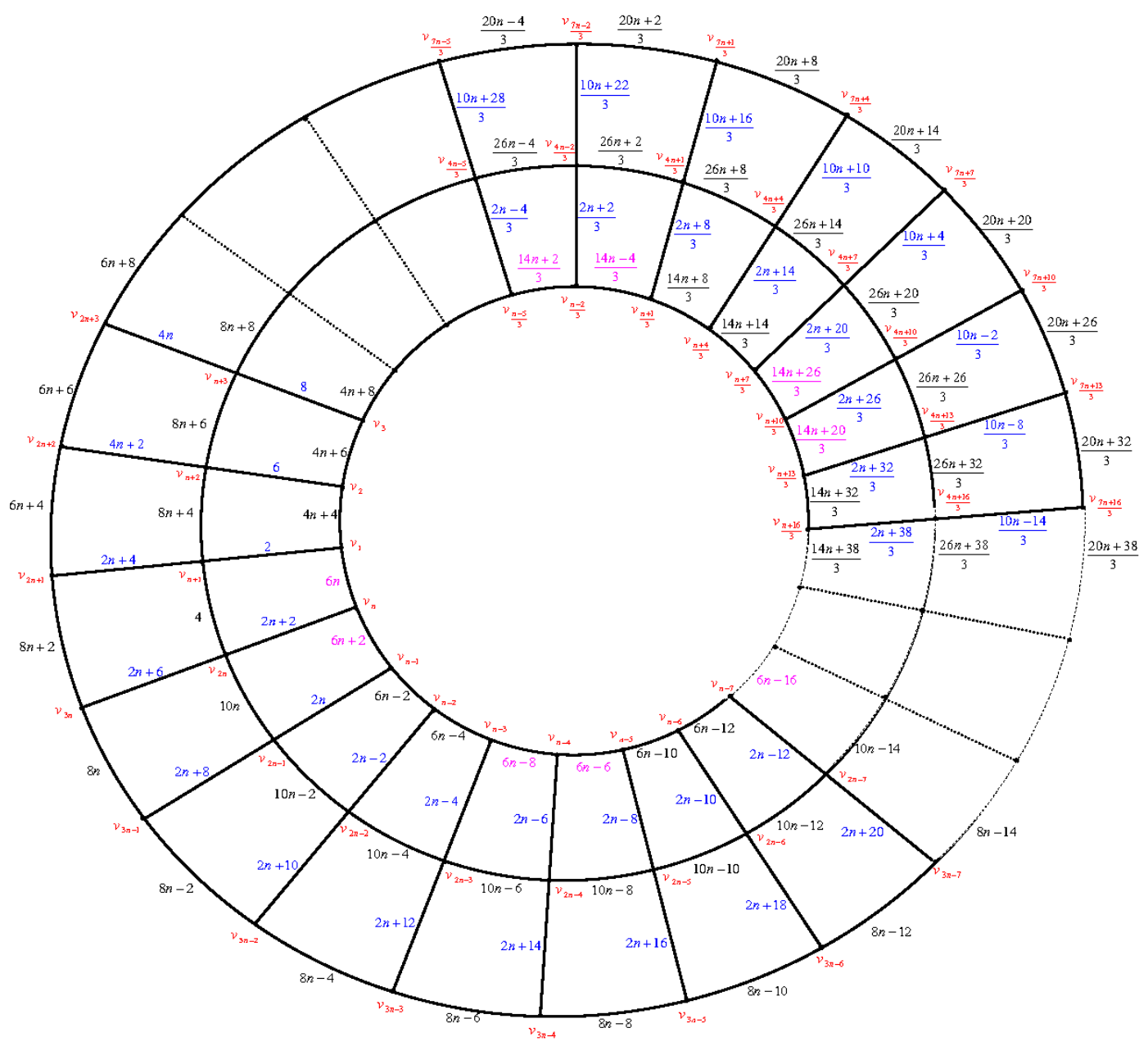

Figure 6. The cylinder grid graph $C_{3, n}, n \equiv 5 \bmod 6$.

First, we label the edges of the paths $P_{3}^{(k)}, 1 \leq k \leq 2$ beginning with the edges of the path $P_{3}^{(1)}$ as the same in case (1). Second, we label the edges of the circles $C_{n}^{(k)}, 1 \leq k \leq 3$ beginning with the edges of the innermost circle $C_{n}^{(1)}$, then the edges of outer circle $C_{n}^{(3)}$, and then the edges of the circle $C_{n}^{(2)}$.

Label the edges of the circle $C_{n}^{(1)}$ as follows: $f\left(v_{1} v_{2}\right)=4 n+4, f\left(v_{2} v_{3}\right)=4 n+6, \ldots, f\left(v_{\frac{n-5}{3}} v_{\frac{n-2}{3}}\right)=$ $\frac{14 n+2}{3}, f\left(v_{\frac{n-2}{3}} v_{\frac{n+1}{3}}\right)=\frac{14 n-4}{3}, f\left(v_{\frac{n+1}{3}} v_{\frac{n+4}{3}}\right)=\frac{14 n+8}{3}, f\left(v_{\frac{n+4}{3}} v_{\frac{n+7}{3}}\right)=\frac{14 n+14}{3}, f\left(v_{\frac{n+7}{3}} v_{\frac{n+10}{3}}\right)=$ $\frac{14 n+26}{3}, f\left(v_{\frac{n+10}{3}} v_{\frac{n+13}{3}}\right)=\frac{14 n+20}{3}, f\left(v_{\frac{n+13}{3}} v_{\frac{n+16}{3}}\right)=\frac{14 n+32}{3}, f\left(v_{\frac{n+16}{3}} v_{\frac{n+19}{3}}\right)=\frac{14 n+38}{3}, f\left(v_{\frac{n+19}{3}} v_{\frac{n+22}{3}}\right)=$ $\frac{14 n+50}{3}, f\left(v_{\frac{n+22}{3}}^{3} v_{\frac{n+25}{3}}^{3}\right)=\frac{14 n+44}{3}, f\left(v_{\frac{n+25}{3}} v_{\frac{n+28}{3}}^{3}\right)=\frac{14 n+56}{3}, f\left(v_{\frac{n+28}{3}}^{3} v_{\frac{n+31}{3}}\right)=\frac{14 n+62}{3}, f\left(v_{\frac{n+31}{3}} v_{\frac{n+34}{3}}\right)=$ $\frac{14 n+74}{3}, f\left(v_{\frac{n+34}{3}}^{3} v_{\frac{n+37}{3}}\right)=\frac{14 n+68}{3}, f\left(v_{\frac{n+37}{3}}^{3} v_{\frac{n+40}{3}}^{3}\right)=\frac{14 n+80}{3}, f\left(v_{\frac{n+40}{3}} v_{\frac{n+43}{3}}^{3}\right)=\frac{14 n+86}{3}, \ldots, f\left(v_{n-13} v_{n-12}\right)=$ $6 n-22, f\left(v_{n-12} v_{n-11}\right)=6 n-24, f\left(v_{n-11} v_{n-10}\right)=6 n-20, f\left(v_{n-10} v_{n-9}\right)=6 n-18, f\left(v_{n-9} v_{n-8}\right)=$ $6 n-14, f\left(v_{n-8} v_{n-7}\right)=6 n-16, f\left(v_{n-7} v_{n-6}\right)=6 n-12, f\left(v_{n-6} v_{n-5}\right)=6 n-10, f\left(v_{n-5} v_{n-4}\right)=6 n-$ $6, f\left(v_{n-4} v_{n-3}\right)=6 n-8, f\left(v_{n-3} v_{n-2}\right)=6 n-4, f\left(v_{n-2} v_{n-1}\right)=6 n-2, f\left(v_{n-1} v_{n}\right)=6 n+2, f\left(v_{n} v_{1}\right)=6 n$.

The labels of corresponding vertices mod10n are as follows: The labels of vertices of the circle $C_{n}^{(1)}: f^{*}\left(v_{1}\right) \equiv 6, f^{*}\left(v_{2}\right) \equiv 8 n+16, f^{*}\left(v_{3}\right) \equiv 8 n+22, \ldots, f^{*}\left(v_{\frac{n}{3}-5}\right) \equiv 10 n-4, f^{*}\left(v_{\frac{n-2}{3}}\right) \equiv$ $0, f^{*}\left(v_{\frac{n+1}{3}}\right) \equiv 4, f^{*}\left(v_{\frac{n+4}{3}}\right) \equiv 12, f^{*}\left(v_{\frac{n+7}{3}}\right) \equiv 20, f^{*}\left(v_{\frac{n+10}{3}}\right) \equiv 24, f^{*}\left(v_{\frac{n+13}{3}}\right) \equiv 28, f^{*}\left(v_{\frac{n+16}{3}}\right) \equiv 36, f^{*}\left(v_{\frac{n}{3}+7}\right) \equiv$ $44, f^{*}\left(v_{\frac{n+19}{3}}\right) \equiv 44, f^{*}\left(v_{\frac{n+22}{3}}\right) \equiv 48, f^{*}\left(v_{\frac{n+25}{3}}\right) \equiv 52, f^{*}\left(v_{\frac{n+28}{3}}\right) \equiv 60, f^{*}\left(v_{\frac{n+31}{3}}\right)^{3} \equiv 68, f^{*}\left(v_{\frac{n+34}{3}}\right) \equiv$ $72, f^{*}\left(v_{\frac{n+37}{3}}^{3}\right) \equiv 76, f^{*}\left(v_{\frac{n+40}{3}}\right) \equiv 84, f^{*}\left(v_{\frac{n+43}{3}}\right) \equiv 92, f^{*}\left(v_{\frac{n+46}{3}}\right) \equiv 96, f^{*}\left(v_{\frac{n+49}{3}}\right) \equiv 100, \ldots, f^{*}\left(v_{n-12}^{3}\right) \equiv$ $4 n-68, f^{*}\left(v_{n-11}\right) \equiv 4 n-64, f^{*}\left(v_{n-10}\right) \equiv 4 n-56, f^{*}\left(v_{n-9}\right) \equiv 4 n-48, f^{*}\left(v_{n-8}^{3}\right) \equiv 4 n-44, f^{*}\left(v_{n-7}\right) \equiv$ $4 n-40, f^{*}\left(v_{n-6}\right) \equiv 4 n-32, f^{*}\left(v_{n-5}\right) \equiv 4 n-24, f^{*}\left(v_{n-4}\right) \equiv 4 n-20, f^{*}\left(v_{n-3}\right) \equiv 4 n-16, f^{*}\left(v_{n-2}\right) \equiv$ $4 n-8, f^{*}\left(v_{n-1}\right) \equiv 4 n, f^{*}\left(v_{n}\right) \equiv 4 n+4$. 
The labels of vertices of the circles $C_{n}^{(2)}$ and $C_{n}^{(3)}$ are the same as in case (1).

Illustration: An e.e.g., 1 . of the cylinder grid graphs $C_{3,25}, C_{3,27}$ and $C_{3,29}$ are shown in Figure 7.

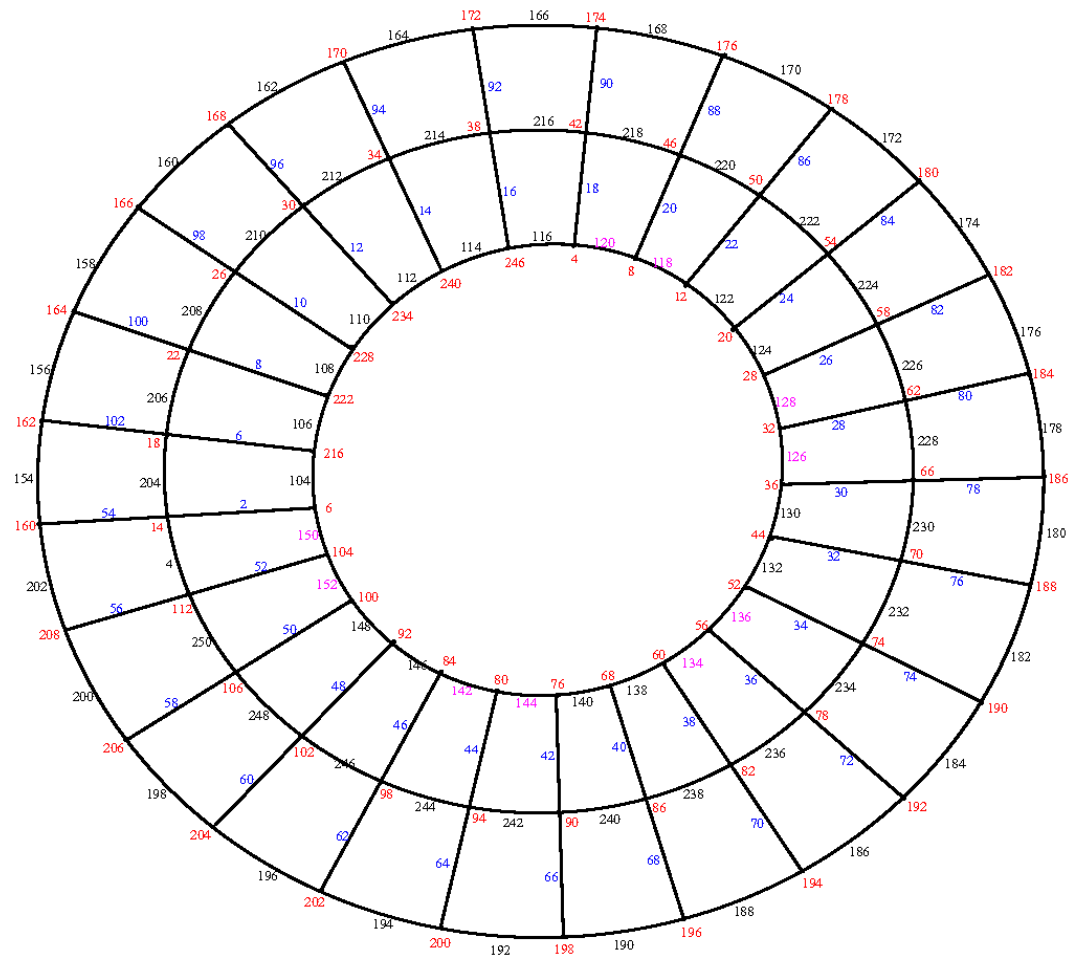

(a) $C_{3,25}$

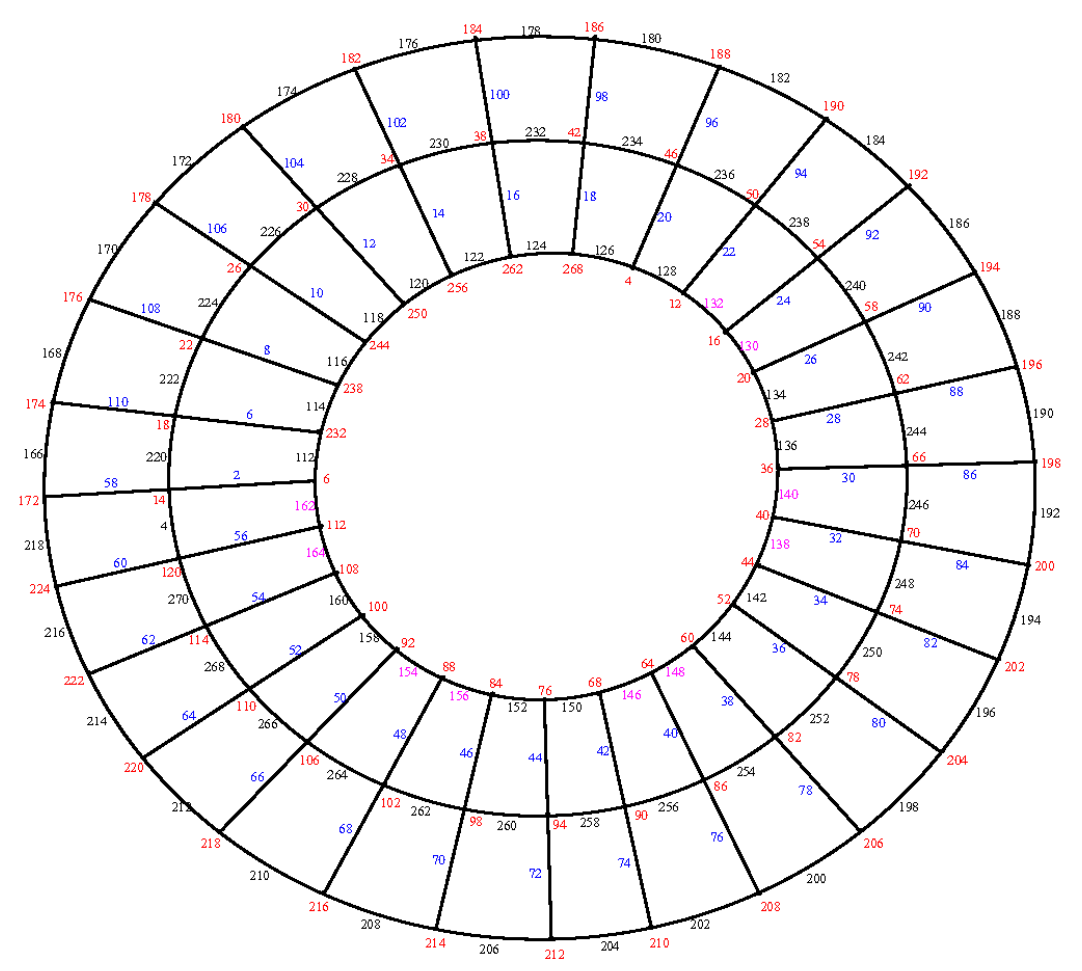

(b) $C_{3,27}$

Figure 7. Cont. 


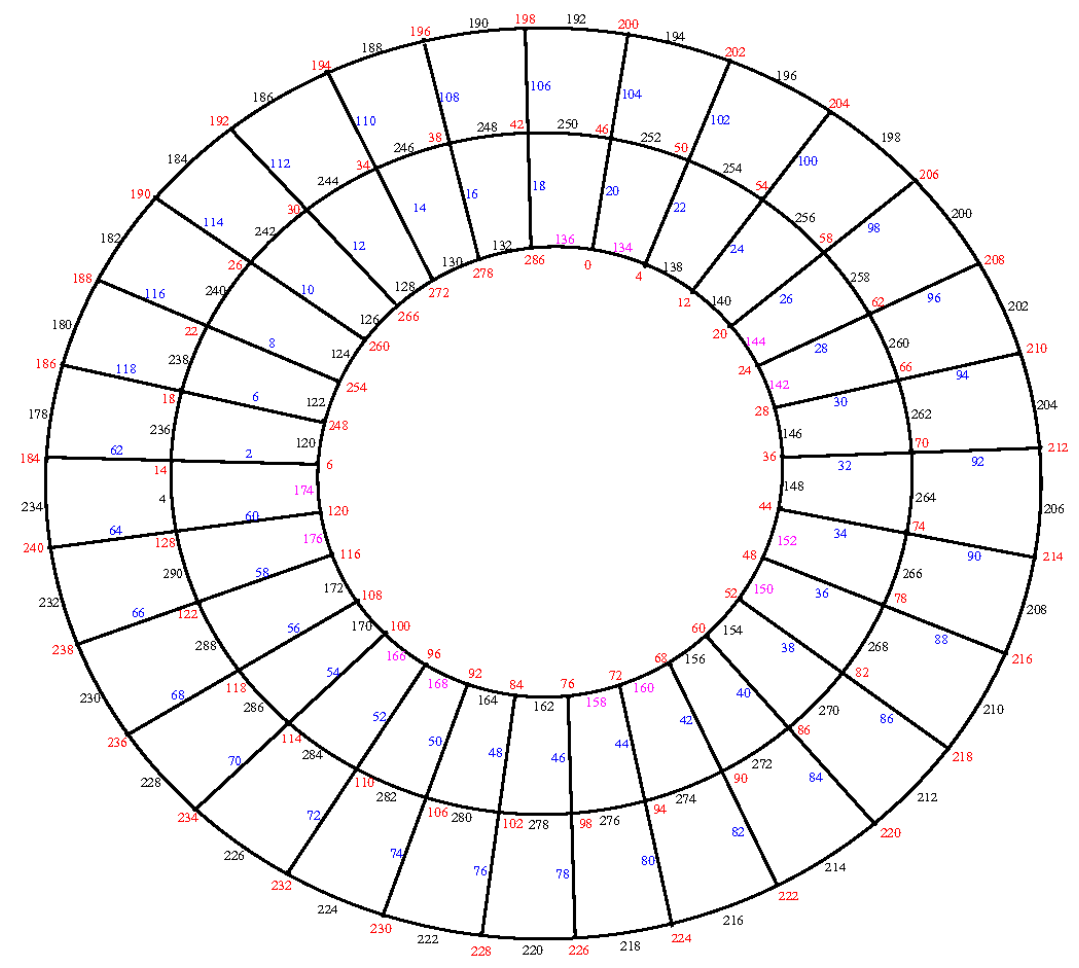

(c) $C_{3,29}$

Figure 7. An e.e.g., l. of the cylinder grid graphs $C_{3,25}, C_{3,27}$ and $C_{3,29}$.

Remark 1. Note that $C_{3,5}$ is an edge even graceful graph but it does not follow the pervious rule (see Figure 8).

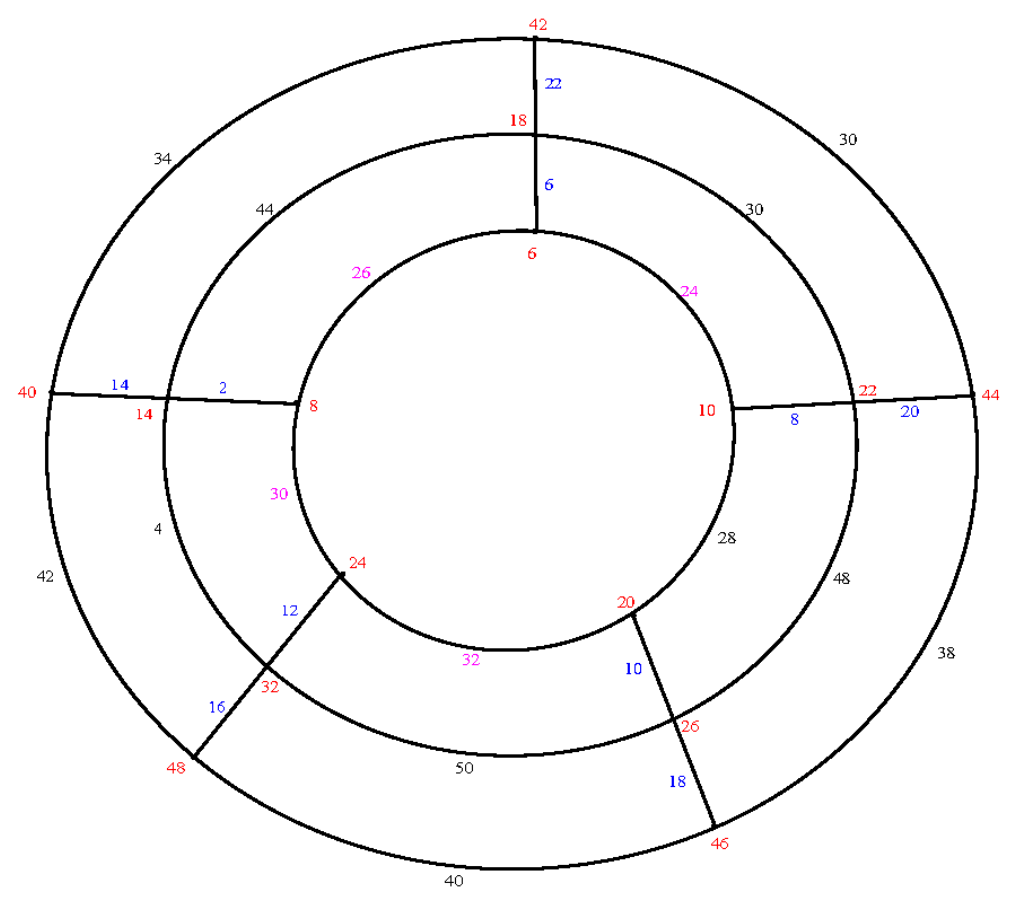

Figure 8. An e.e.g., 1. of the cylinder grid graph $C_{3,5}$. 
Theorem 3. If $m$ is an odd positive integer greater than 3 and $n$ is an even positive integer, $n \geq 2$, then the cylinder grid graph $C_{m, n}$, is an edge even graceful graph.

Proof. Using standard notation $p=\left|V\left(C_{m, n}\right)\right|=m n, q=\left|E\left(C_{m, n}\right)\right|=2 m n-n$ and $r=\max (p, q)=$ $2 m n-n$ and $f: E\left(C_{m, n}\right) \rightarrow\{2,4,6, \ldots, 4 m n-2 n-2\}$.

Let the cylinder grid graph $C_{m, n}$ be as in Figure 9. There are six cases:

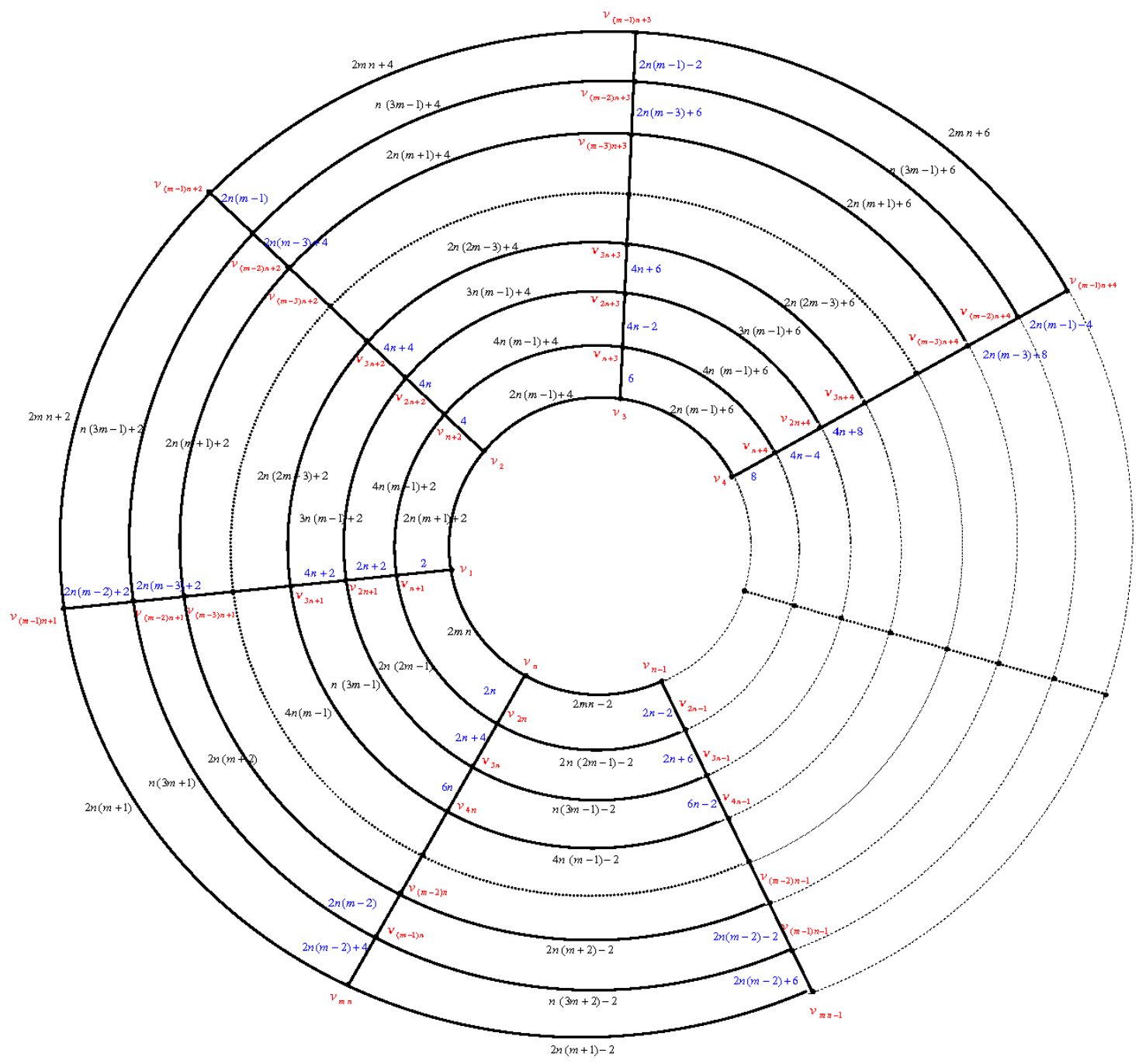

Figure 9. The cylinder grid graph $C_{m, n}, m$ is odd greater than 3 and $n \geq 2$.

Case (1): $n \equiv$ Omod12. First, we label the edges of the paths $P_{m}^{(k)}, 1 \leq k \leq n$ beginning with the edges of the path $P_{m}^{(1)}$ as follows: Move clockwise to label the edges $v_{1} v_{n+1}, v_{2} v_{n+2}, v_{3} v_{n+3}, \ldots, v_{n-1} v_{2 n-1}, v_{n} v_{2 n}$ by $2,4,6, \ldots, 2 n-2,2 n$, then move anticlockwise to label the edges $v_{n+1} v_{n+2}, v_{2 n} v_{3 n}, v_{2 n-1} v_{3 n-1}, \ldots, v_{n+3} v_{2 n+3}, v_{n+2} v_{2 n+2}$ by $2 n+2,2 n+4,2 n+6, \ldots, 4 n-2,4 n$, then move clockwise to label the edges $v_{2 n+1} v_{3 n+1}, v_{2 n+2} v_{3 n+2}, v_{2 n+3} v_{3 n+3}, \ldots, v_{3 n-1} v_{4 n-1}, v_{3 n} v_{4 n}$ by $4 n+2,4 n+4,4 n+6, \ldots, 6 n-2,6 n$ and so on.

Finally, move anticlockwise to label the edges $v_{(m-2) n+1} v_{(m-1) n+1}, v_{(m-1) n} v_{m n}, v_{(m-1) n-1} v_{m n-1}, \ldots$, $v_{(m-2) n+3} v_{(m-1) n+3}, v_{(m-2) n+2} v_{m(n-1)+2}$ by $2 n(m-2)+2,2 n(m-2)+4,2 n(m-2)+6, \ldots, 2 n(m-1)-$ $2,2 n(m-1)$. 
Secondly, we label the edges of the circles $C_{n}^{(k)}, 1 \leq k \leq m$ beginning with the edges of the inner most circle $C_{n}^{(1)}$, then the edges of outer circle $C_{n}^{(m)}$, then the edges of the circles $C_{m}^{(m-1)}, C_{m}^{(m-3)}, \ldots, C_{m}^{(2)}$. Finally, we label the edges of the circles $C_{m}^{(m-1)}, C_{m}^{(m-3)}, \ldots, C_{m}^{(2)}$.

Label the edges of the circle $C_{n}^{(1)}$ as follows: $f\left(v_{1} v_{2}\right)=2 n(m-1)+2, f\left(v_{2} v_{3}\right)=2 n(m-1)+$ $6, f\left(v_{3} v_{4}\right)=2 n(m-1)+4, f\left(v_{4} v_{5}\right)=2 n(m-1)+8, f\left(v_{5} v_{6}\right)=2 n(m-1)+10, f\left(v_{6} v_{7}\right)=2 n(m-$ $1)+14, f\left(v_{7} v_{8}\right)=2 n(m-1)+12, f\left(v_{8} v_{9}\right)=2 n(m-1)+16, f\left(v_{9} v_{10}\right)=2 n(m-1)+18, f\left(v_{10} v_{11}\right)=$ $2 n(m-1)+22, f\left(v_{11} v_{12}\right)=2 n(m-1)+20, f\left(v_{12} v_{13}\right)=2 n(m-1)+24, \ldots, f\left(v_{n-7} v_{n-6}\right)=2 m n-$ $14, f\left(v_{n-6} v_{n-5}\right)=2 m n-10, f\left(v_{n-5} v_{n-4}\right)=2 m n-12, f\left(v_{n-4} v_{n-3}\right)=2 m n-8, f\left(v_{n-3} v_{n-2}\right)=2 m n-$ $2, f\left(v_{n-2} v_{n-1}\right)=2 m n-6, f\left(v_{n-1} v_{n}\right)=2 m n, f\left(v_{n} v_{1}\right)=2 m n-4$.

Label the edges of the circle $C_{n}^{(m)}$ as follows: $f\left(v_{(m-1) n+i} v_{(m-1) n+i+1}\right)=2 m n+$ $2 i, f\left(v_{m n} v_{(m-1) n+1}\right)=2 n(m+1), 1 \leq i \leq n-1$.

Label the edges of the circle $C_{n}^{(m-2)}$ as follows: $f\left(v_{(m-3) n+i} v_{(m-3) n+i+1}\right)=2 n(m+1)+$ $2 i, f\left(v_{(m-2) n} v_{(m-3) n+1}\right)=2 n(m+2), 1 \leq i \leq n-1$.

Label the edges of the circle $C_{n}^{(m-4)}$ as follows: $f\left(v_{(m-5) n+i} v_{(m-5) n+i+1}\right)=2 n(m+2)+$ $2 i, f\left(v_{(m-4) n} v_{(m-5) n+1}\right)=2 n(m+3), 1 \leq i \leq n-1$, and so on.

Label the edges of the circle $C_{n}^{(3)}$ as follows: $f\left(v_{2 n+i} v_{2 n+i+1}\right)=3 n(m-1)+2 i, f\left(v_{3 n} v_{2 n+1}\right)=$ $n(3 m-1), 1 \leq i \leq n-1$,

Label the edges of the circle $C_{n}^{(m-1)}$ as follows: $f\left(v_{(m-2) n+i} v_{(m-2) n+i+1}\right)=n(3 m-1)+$ $2 i, f\left(v_{(m-1) n} v_{(m-1) n+1}\right)=n(3 m+1)-1,1 \leq i \leq n-1$,

Label the edges of the circle $C_{n}^{(m-3)}$ as follows: $f\left(v_{(m-4) n+i} v_{(m-4) n+i+1}\right)=n(3 m+2)+$ $2 i, f\left(v_{(m-3) n} v_{(m-4) n+1}\right)=3 n(m+1), 1 \leq i \leq n-1, \ldots$, and so on.

Label the edges of the circle $C_{n}^{(4)}$ as follows: $f\left(v_{3 n+i} v_{3 n+i+1}\right)=2 n(2 m-3)+2 i, f\left(v_{4 n} v_{3 n+1}\right)=$ $4 n(m-1), 1 \leq i \leq n-1$,

Label the edges of $C_{n}^{(2)}$ as follows: $f\left(v_{n+i} v_{n+i+1}\right)=4 n(m-1)+2 i, f\left(v_{2 n} v_{2 n+1}\right)=2 n(m-1), 1 \leq$ $i \leq n-1$,

Thus, the labels of corresponding vertices $\bmod (4 m n-2 n)$ will be:

The label the vertices of $C_{n}^{(1)}$ are: $f^{*}\left(v_{1}\right) \equiv 0 ; f^{*}\left(v_{2}\right) \equiv 4 m n-4 n+12 ; f^{*}\left(v_{3}\right) \equiv 4 m n-4 n+$ $16 ; f^{*}\left(v_{4}\right) \equiv 4 m n-4 n+20 ; f^{*}\left(v_{5}\right) \equiv 4 m n-4 n+28 ; f^{*}\left(v_{6}\right) \equiv 4 m n-4 n+36 ; f^{*}\left(v_{7}\right) \equiv 4 m n-4 n+$ $40 ; f^{*}\left(v_{8}\right) \equiv 4 m n-4 n+44 ; f^{*}\left(v_{9}\right) \equiv 4 m n-4 n+52 ; f^{*}\left(v_{10}\right) \equiv 4 m n-4 n+60 ; f^{*}\left(v_{11}\right) \equiv 4 m n-4 n+$ $64 ; f^{*}\left(v_{12}\right) \equiv 4 m n-4 n+68 ; \ldots ; f^{*}\left(v_{n-6}\right) \equiv 4 n-36 ; f^{*}\left(v_{n-5}\right) \equiv 4 n-32 ; f^{*}\left(v_{n-4}\right) \equiv 4 n-28 ; f^{*}\left(v_{n-3}\right) \equiv$ $4 n-16 ; f^{*}\left(v_{n-2}\right) \equiv 4 n-12 ; f^{*}\left(v_{n-1}\right) \equiv 4 n-8 ; f^{*}\left(v_{n}\right) \equiv 4 n-4$.

The label the vertices of $C_{n}^{(2)}, C_{n}^{(3)}, C_{n}^{(4)}, \ldots, C_{n}^{(m-2)}, C_{n}^{(m-1)}, C_{n}^{(m)}$ respectively are: $f^{*}\left(v_{n+i}\right) \equiv$ $4 i+2 ; f^{*}\left(v_{2 n+i}\right) \equiv 2 m n+4 n+4 i+2 ; f^{*}\left(v_{3 n+i}\right) \equiv 4 n+4 i+2 ; \ldots ; f^{*}\left(v_{(m-3) n+i}\right) \equiv 4 m n-6 n+4 i+2 ;$ $f^{*}\left(v_{(m-2) n+i}\right) \equiv 2 m n-6 n+4 i+2 ; f^{*}\left(v_{(m-1) n+i}\right) \equiv 2 m n+2 i+2,1 \leq i \leq n$.

Case (2): $n \equiv 2 \bmod 12, n \neq 2$.

First, we label the edges of the paths $P_{m}^{(k)}, 1 \leq k \leq n$ begin with the edges of the path $P_{m}^{(1)}$ as the same in case (1).

Secondly, we label the edges of the circles $C_{n}^{(k)}, 1 \leq k \leq m$ begin with the edges of the inner most circle $C_{n}^{(1)}$, then the edges of outer circle $C_{n}^{(m)}$, then the edges of the circles $C_{n}^{(m-2)}, C_{n}^{(m-4)}, \ldots, C_{n}^{(3)}$.

Finally, we label the edges of the circles $C_{m}^{(m-1)}, C_{m}^{(m-3)}, \ldots, C_{m}^{(2)}$.

Label the edges of the circle $C_{n}^{(1)}$ as follows: $f\left(v_{1} v_{2}\right)=2 n(m-1)+2, f\left(v_{2} v_{3}\right)=2 n(m-1)+$ $6, f\left(v_{3} v_{4}\right)=2 n(m-1)+4, f\left(v_{4} v_{5}\right)=2 n(m-1)+8, f\left(v_{5} v_{6}\right)=2 n(m-1)+10, f\left(v_{6} v_{7}\right)=2 n(m-$ $1)+14, f\left(v_{7} v_{8}\right)=2 n(m-1)+12, f\left(v_{8} v_{9}\right)=2 n(m-1)+16, f\left(v_{9} v_{10}\right)=2 n(m-1)+18, f\left(v_{10} v_{11}\right)=$ $2 n(m-1)+22, f\left(v_{11} v_{12}\right)=2 n(m-1)+20, f\left(v_{12} v_{13}\right)=2 n(m-1)+24, \ldots, f\left(v_{n-9} v_{n-8}\right)=2 m n-$ $18, f\left(v_{n-8} v_{n-7}\right)=2 m n-14, f\left(v_{n-7} v_{n-6}\right)=2 m n-16, f\left(v_{n-6} v_{n-5}\right)=2 m n-12, f\left(v_{n-5} v_{n-4}\right)=$ $2 m n-10, f\left(v_{n-4} v_{n-3}\right)=2 m n-6, f\left(v_{n-3} v_{n-2}\right)=2 m n-8, f\left(v_{n-2} v_{n-1}\right)=2 m n-4, f\left(v_{n-1} v_{n}\right)=$ $2 m n-2, f\left(v_{n} v_{1}\right)=2 m n$. 
Label the edges of the circle $C_{n}^{(2)}$ as follows: $f\left(v_{n+1} v_{n+2}\right)=4 n(m-1)+4, f\left(v_{n+2} v_{n+3}\right)=$ $4 n(m-1)+2, f\left(v_{n+3} v_{n+4}\right)=4 n(m-1)+8, f\left(v_{n+4} v_{n+5}\right)=4 n(m-1)+6, f\left(v_{n+i} v_{n+i+1}\right)=4 n(m-$ 1) $+2 i, 6 \leq i \leq n-2, f\left(v_{2 n-1} v_{2 n}\right)=2 n(2 m-1), f\left(v_{2 n} v_{n+1}\right)=2 n(2 m-1)-2$. Label the edges of $C_{n}^{(m)}, C_{n}^{(m-2)}, C_{n}^{(m-4)}, \ldots, C_{n}^{(3)}$ and $C_{n}^{(m-1)}, C_{n}^{(m-3)}, C_{n}^{(m-5)}, \ldots, C_{n}^{(4)}$ as in case (1).

Thus, the labels of corresponding vertices $\bmod (4 m n-2 n)$ will be:

The label the vertices of $C_{n}^{(1)}$ are: $f^{*}\left(v_{1}\right) \equiv 4, f^{*}\left(v_{2}\right) \equiv 4 m n-4 n+12, f^{*}\left(v_{3}\right) \equiv 4 m n-$ $4 n+16, f^{*}\left(v_{4}\right) \equiv 4 m n-4 n+20, f^{*}\left(v_{5}\right) \equiv 4 m n-4 n+28, f^{*}\left(v_{6}\right) \equiv 4 m n-4 n+36, f^{*}\left(v_{7}\right) \equiv$ $4 m n-4 n+40, f^{*}\left(v_{8}\right) \equiv 4 m n-4 n+44, f^{*}\left(v_{9}\right) \equiv 4 m n-4 n+52, f^{*}\left(v_{10}\right) \equiv 4 m n-4 n+60, f^{*}\left(v_{11}\right) \equiv$ $4 m n-4 n+64, f^{*}\left(v_{12}\right) \equiv 4 m n-4 n+68, f^{*}\left(v_{13}\right) \equiv 4 m n-4 n+76, \ldots, f^{*}\left(v_{n-8}\right) \equiv 4 n-48, f^{*}\left(v_{n-7}\right) \equiv$ $4 n-44, f^{*}\left(v_{n-6}\right) \equiv 4 n-40, f^{*}\left(v_{n-5}\right) \equiv 4 n-32, f^{*}\left(v_{n-4}\right) \equiv 4 n-24, f^{*}\left(v_{n-3}\right) \equiv 4 n-20, f^{*}\left(v_{n-2}\right) \equiv$ $4 n-16, f^{*}\left(v_{n-1}\right) \equiv 4 n-8, f^{*}\left(v_{n}\right) \equiv 4 n-2$.

The label the vertices of the circle $C_{n}^{(2)}$ are: $f^{*}\left(v_{n+1}\right) \equiv 6, f^{*}\left(v_{n+2}\right) \equiv 10, f^{*}\left(v_{n+3}\right) \equiv 14, f^{*}\left(v_{n+4}\right) \equiv$ $18, f^{*}\left(v_{n+5}\right) \equiv 20, f^{*}\left(v_{n+i}\right) \equiv 4 i+2,6 \leq i \leq n-2, f^{*}\left(v_{2 n-1}\right) \equiv 4 n, f^{*}\left(v_{2 n}\right) \equiv 4 n+2$.

The label the vertices of $C_{n}^{(3)}, C_{n}^{(4)}, \ldots, C_{n}^{(m-2)}, C_{n}^{(m-1)}, C_{n}^{(m)}$ respectively are as the same as in case (1).

Remark 2. In case $n=2$. Let the edges of the cylinder grid graph $C_{m, 2}$ are labeled as shown in Figure 10 . The corresponding labels of vertices $\bmod (8 m-4)$ are as follows: $f^{*}\left(v_{1}\right) \equiv 8, f^{*}\left(v_{2 i+1}\right) \equiv 4 m+8 i+4,1 \leq$ $i \leq \frac{m-3}{2}, f^{*}\left(v_{2 i}\right) \equiv 8 i+6,1 \leq i \leq \frac{m-1}{2} ; f^{*}\left(v^{\prime}{ }_{1}\right) \equiv 12, f^{*}\left(v^{\prime}{ }_{2}\right) \equiv 20, f^{*}\left(v^{\prime}{ }_{2 i+1}\right) \equiv 4 m+8 i+18,1 \leq i \leq$ $\frac{m-3}{2}, f^{*}\left(v^{\prime}{ }_{2 i}\right) \equiv 8 i+10,2 \leq i \leq \frac{m-1}{2}$.

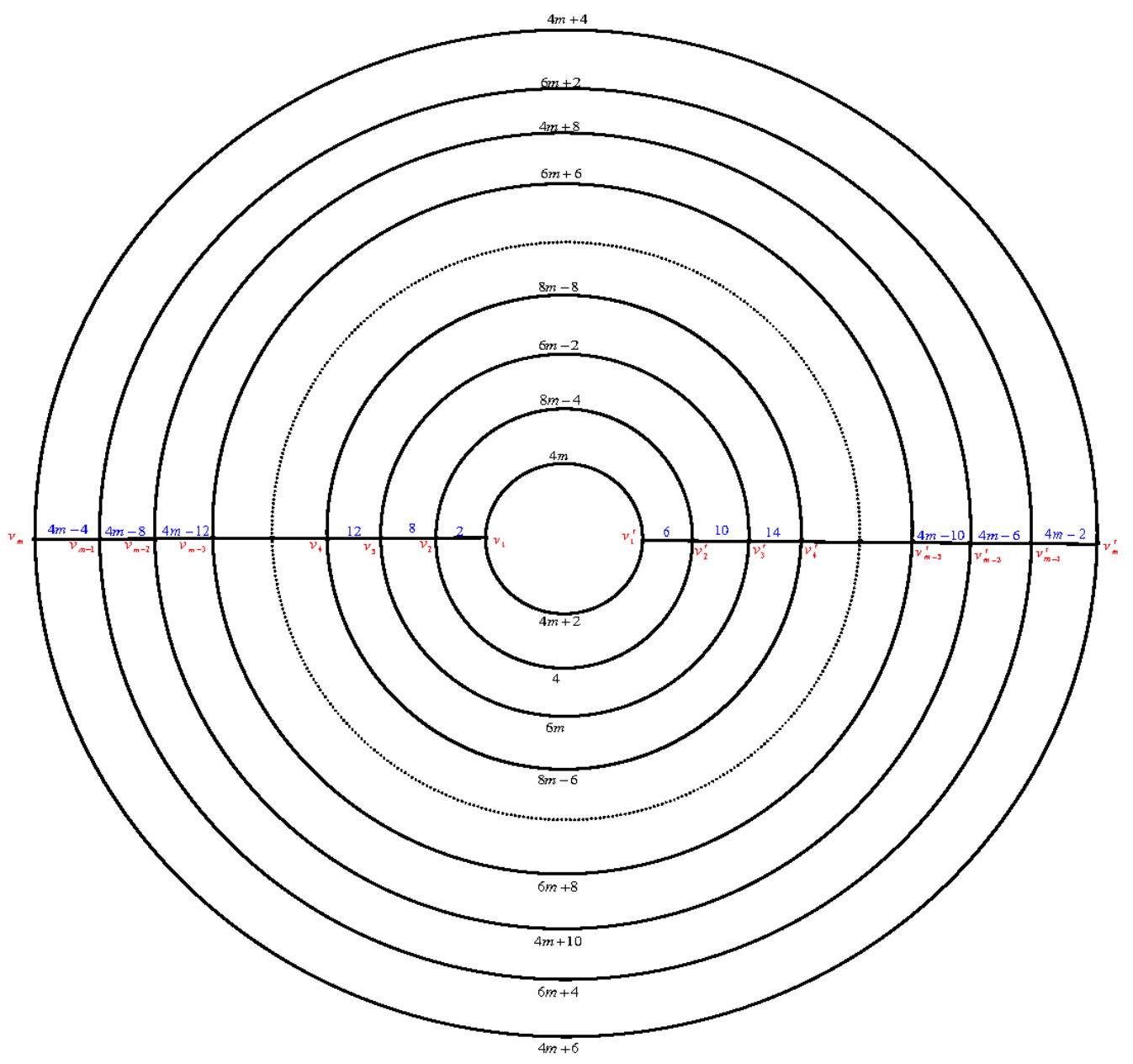

Figure 10. The cylinder grid graph $C_{m, 2}$. 
Case (3): $n \equiv 4 \bmod 12$.

First we label the edges of the paths $P_{m}^{(k)}, 1 \leq k \leq n$ begin with the edges of the path $P_{m}^{(1)}$ as the same in case (1).

Second we label the edges of the circles $C_{n}^{(k)}, 1 \leq k \leq m$ begin with the edges of the inner most circle $C_{n}^{(1)}$, then the edges of outer circle $C_{n}^{(m)}$, then the edges of the circles $C_{n}^{(m-2)}, C_{n}^{(m-4)}, \ldots, C_{n}^{(3)}$.

Finally we label the edges of the circles $C_{m}^{(m-1)}, C_{m}^{(m-3)}, \ldots, C_{m}^{(2)}$.

Label the edges of the circle $C_{n}^{(1)}$ as follows: $f\left(v_{1} v_{2}\right)=2 n(m-1)+2, f\left(v_{2} v_{3}\right)=2 n(m-1)+$ $6, f\left(v_{3} v_{4}\right)=2 n(m-1)+4, f\left(v_{4} v_{5}\right)=2 n(m-1)+8, f\left(v_{5} v_{6}\right)=2 n(m-1)+10, f\left(v_{6} v_{7}\right)=2 n(m-$ $1)+14, f\left(v_{7} v_{8}\right)=2 n(m-1)+12, f\left(v_{8} v_{9}\right)=2 n(m-1)+16, f\left(v_{9} v_{10}\right)=2 n(m-1)+18, f\left(v_{10} v_{11}\right)=$ $2 n(m-1)+22, f\left(v_{11} v_{12}\right)=2 n(m-1)+20, f\left(v_{12} v_{13}\right)=2 n(m-1)+24, \ldots, f\left(v_{n-8} v_{n-7}\right)=2 m n-$ $16, f\left(v_{n-7} v_{n-6}\right)=2 m n-14, f\left(v_{n-6} v_{n-5}\right)=2 m n-10, f\left(v_{n-5} v_{n-4}\right)=2 m n-12, f\left(v_{n-4} v_{n-3}\right)=2 m n-$ $8, f\left(v_{n-3} v_{n-2}\right)=2 m n-2, f\left(v_{n-2} v_{n-1}\right)=2 m n-6, f\left(v_{n-1} v_{n}\right)=2 m n$, and $f\left(v_{n} v_{1}\right)=2 m n-4$.

Label the edges of $C_{n}^{(m)}, C_{n}^{(m-2)}, C_{n}^{(m-4)}, \ldots, C_{n}^{(3)}$ and $C_{n}^{(m-1)}, C_{n}^{(m-3)}, C_{n}^{(m-5)}, \ldots, C_{n}^{(4)}, C_{n}^{(2)}$ as in case (1).

Thus we have the labels of corresponding vertices of the circle $C_{n}^{(1)} \bmod (4 m n-2 n)$ will be: $f^{*}\left(v_{1}\right) \equiv 0, f^{*}\left(v_{2}\right) \equiv 4 m n-4 n+12, f^{*}\left(v_{3}\right) \equiv 4 m n-4 n+16, f^{*}\left(v_{4}\right) \equiv 4 m n-4 n+20, f^{*}\left(v_{5}\right) \equiv 4 m n-$ $4 n+28, f^{*}\left(v_{6}\right) \equiv 4 m n-4 n+36, f^{*}\left(v_{7}\right) \equiv 4 m n-4 n+40, f^{*}\left(v_{8}\right) \equiv 4 m n-4 n+44, f^{*}\left(v_{9}\right) \equiv 4 m n-$ $4 n+52, f^{*}\left(v_{10}\right) \equiv 4 m n-4 n+60, f^{*}\left(v_{11}\right) \equiv 4 m n-4 n+64, f^{*}\left(v_{12}\right) \equiv 4 m n-4 n+68, f^{*}\left(v_{13}\right) \equiv 4 m n-$ $4 n+76, \ldots, f^{*}\left(v_{n-7}\right) \equiv 4 n-44, f^{*}\left(v_{n-6}\right) \equiv 4 n-36, f^{*}\left(v_{n-5}\right) \equiv 4 n-32, f^{*}\left(v_{n-4}\right) \equiv 4 n-28, f^{*}\left(v_{n-3}\right) \equiv$ $4 n-16, f^{*}\left(v_{n-2}\right) \equiv 4 n-12, f^{*}\left(v_{n-1}\right) \equiv 4 n-8, f^{*}\left(v_{n}\right) \equiv 4 n-4$.

The label the vertices of $C_{n}^{(2)}, C_{n}^{(3)}, C_{n}^{(4)}, \ldots, C_{n}^{(m-2)}, C_{n}^{(m-1)}, C_{n}^{(m)}$ respectively are as same in case (1)

Remark 3. In case $n=4$. Let the the edges of the cylinder grid graph $C_{m, 4}$ are labeled as shown in Figure 11 . The corresponding labels of vertices $\bmod (16 m-8)$ are as follows: $f^{*}\left(v_{1}\right) \equiv 6, f^{*}\left(v_{2}\right) \equiv 8, f^{*}\left(v_{3}\right) \equiv 16, f^{*}\left(v_{4}\right) \equiv$ $20 ; f^{*}\left(v_{4 i+1}\right) \equiv 4 i+10,1 \leq i \leq 3, f^{*}\left(v_{8}\right) \equiv 28 ; f^{*}\left(v_{8 k+i}\right) \equiv 8 m+4 i+16 k-10,1 \leq i \leq 4,1 \leq k \leq$ $\frac{m-5}{2} ; f^{*}\left(v_{4 m-11}\right) \equiv 0, f^{*}\left(v_{4 m-10}\right) \equiv 2, f^{*}\left(v_{4 m-9}\right) \equiv 4, f^{*}\left(v_{4 m-8}\right) \equiv 10, f^{*}\left(v_{8 k+4+i}\right) \equiv 4 i+16 k+10,1 \leq i \leq$ $4,1 \leq k \leq \frac{m-3}{2}$.

Case (4): $n \equiv 6 \bmod 12$.

First, we label the edges of the paths $P_{m}^{(k)}, 1 \leq k \leq n$ begin with the edges of the path $P_{m}^{(1)}$ as the same in case (1).

Secondly, we label the edges of the circles $C_{n}^{(k)}, 1 \leq k \leq m$ begin with the edges of the inner most circle $C_{n}^{(1)}$, then the edges of outer circle $C_{n}^{(m)}$, then the edges of the circles $C_{n}^{(m-2)}, C_{n}^{(m-4)}, \ldots, C_{n}^{(3)}$.

Finally, we label the edges of the circles $C_{m}^{(m-1)}, C_{m}^{(m-3)}, \ldots, C_{m}^{(2)}$.

Label the edges of the circle $C_{n}^{(1)}$ as follows: $f\left(v_{1} v_{2}\right)=2 n(m-1)+2, f\left(v_{2} v_{3}\right)=2 n(m-1)+$ $6, f\left(v_{3} v_{4}\right)=2 n(m-1)+4, f\left(v_{4} v_{5}\right)=2 n(m-1)+8, f\left(v_{5} v_{6}\right)=2 n(m-1)+10, f\left(v_{6} v_{7}\right)=2 n(m-$ $1)+14, f\left(v_{7} v_{8}\right)=2 n(m-1)+12, f\left(v_{8} v_{9}\right)=2 n(m-1)+16, f\left(v_{9} v_{10}\right)=2 n(m-1)+18, f\left(v_{10} v_{11}\right)=$ $2 n(m-1)+22, f\left(v_{11} v_{12}\right)=2 n(m-1)+20, f\left(v_{12} v_{13}\right)=2 n(m-1)+24, \ldots, f\left(v_{n-9} v_{n-8}\right)=2 m n-$ $18, f\left(v_{n-8} v_{n-7}\right)=2 m n-14, f\left(v_{n-7} v_{n-6}\right)=2 m n-16, f\left(v_{n-6} v_{n-5}\right)=2 m n-12, f\left(v_{n-5} v_{n-4}\right)=2 m n-$ $10, f\left(v_{n-4} v_{n-3}\right)=2 m n-6, f\left(v_{n-3} v_{n-2}\right)=2 m n-8, f\left(v_{n-2} v_{n-1}\right)=2 m n-4, f\left(v_{n-1} v_{n}\right)=2 m n+2$, $f\left(v_{n} v_{1}\right)=2 m n-2$.

Label the edges of $C_{n}^{(m-4)}, \ldots, C_{n}^{(3)}$ and $C_{n}^{(m-1)}, C_{n}^{(m-3)}, C_{n}^{(m-5)}, \ldots, C_{n}^{(4)}, C_{n}^{(2)}$ as in case (1).

Label the edges of the circle $C_{n}^{(m-2)}$ as follows: $f\left(v_{(m-3) n+1} v_{(m-3) n+2}\right)=2 n(m+$ 2), $f\left(v_{(m-3) n+i} v_{(m-3) n+i+1}\right)=2 n(m+1)+2 i, 2 \leq i \leq n-1, f\left(v_{(m-2) n} v_{(m-3) n+1}\right)=2 n(m+2)+2$.

Label the edges of the circle $C_{n}^{(m)}$ as follows: $f\left(v_{(m-1) n+1} v_{(m-1) n+2}\right)=$ $2 m n, f\left(v_{(m-1) n+i} v_{(m-1) n+i+1}\right)=2 m n+2 i, 2 \leq i \leq n-1, f\left(v_{(m-2) n} v_{(m-3) n+1}\right)=2 n(m+2)$. 


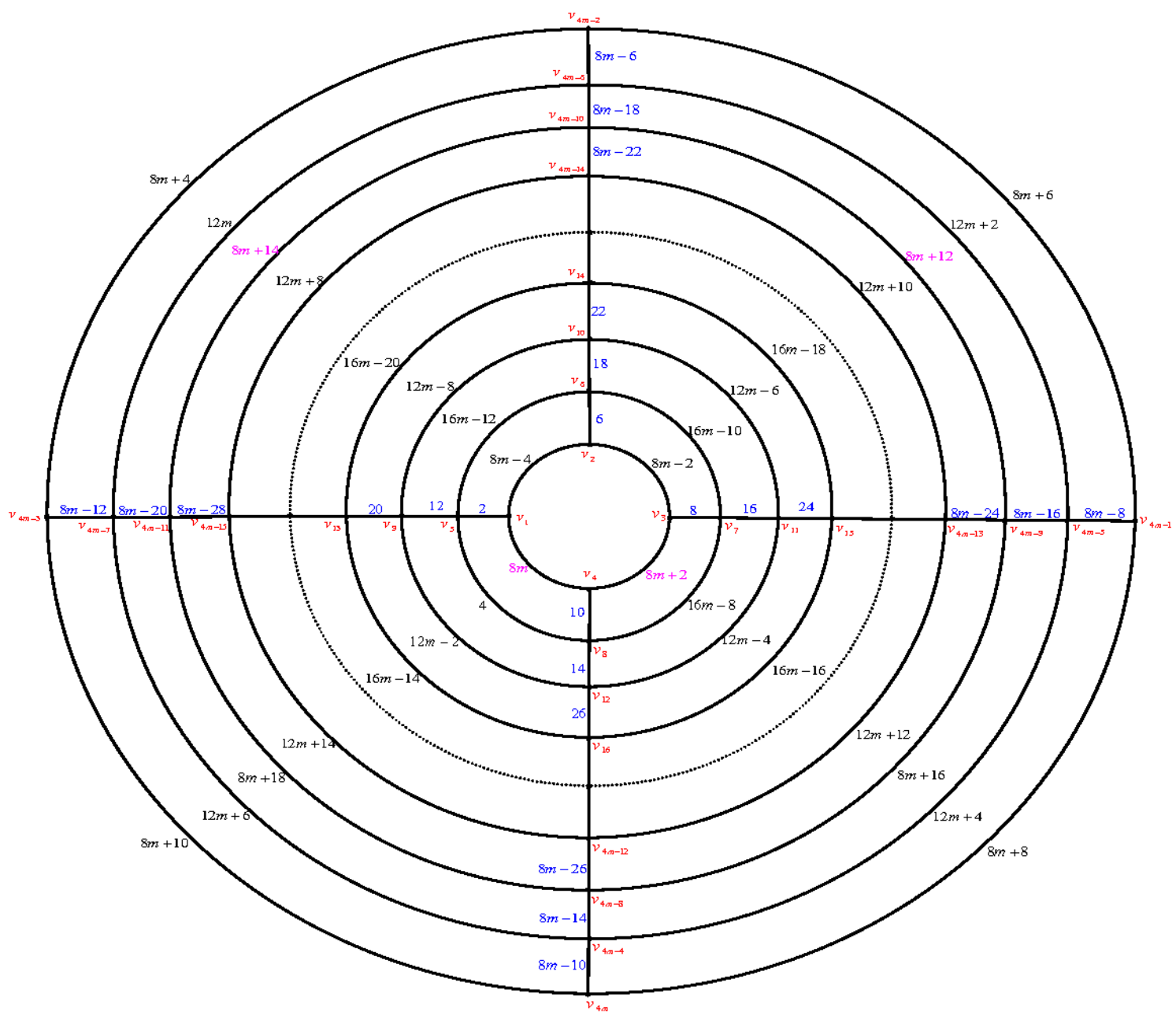

Figure 11. An e.e.g., 1 . of the cylinder grid graph $C_{m, 4}$.

Thus we have the labels of corresponding vertices $\bmod (4 m n-2 n)$ will be:

The labels the vertices of the circle $C_{n}^{(1)}$ are: $f^{*}\left(v_{1}\right) \equiv 2, f^{*}\left(v_{2}\right) \equiv 4 m n-4 n+12, f^{*}\left(v_{3}\right) \equiv$ $4 m n-4 n+16, f^{*}\left(v_{4}\right) \equiv 4 m n-4 n+20, f^{*}\left(v_{5}\right) \equiv 4 m n-4 n+28, f^{*}\left(v_{6}\right) \equiv 4 m n-4 n+36, f^{*}\left(v_{7}\right) \equiv 4 m n-$ $4 n+40, f^{*}\left(v_{8}\right) \equiv 4 m n-4 n+44, f^{*}\left(v_{9}\right) \equiv 4 m n-4 n+52, f^{*}\left(v_{10}\right) \equiv 4 m n-4 n+60, f^{*}\left(v_{11}\right) \equiv 4 m n-4 n+$ $64, f^{*}\left(v_{12}\right) \equiv 4 m n-4 n+68, \ldots, f^{*}\left(v_{n-8}\right) \equiv 4 n-48, f^{*}\left(v_{n-7}\right) \equiv 4 n-44, f^{*}\left(v_{n-6}\right) \equiv 4 n-40, f^{*}\left(v_{n-5}\right) \equiv$ $4 n-32, f^{*}\left(v_{n-4}\right) \equiv 4 n-24, f^{*}\left(v_{n-3}\right) \equiv 4 n-20, f^{*}\left(v_{n-2}\right) \equiv 4 n-16, f^{*}\left(v_{n-1}\right) \equiv 4 n-4 ; f^{*}\left(v_{n}\right) \equiv 4 n$.

The labels the vertices of the circle $C_{n}^{(m-2)}$ are: $f^{*}\left(v_{(m-3) n+1}\right) \equiv 4 m n-6 n+6 ; f^{*}\left(v_{(m-3) n+2}\right) \equiv$ $4 m n-6 n+8 ; f^{*}\left(v_{(m-3) n+i}\right) \equiv 4 m n-6 n+4 i+2,3 \leq i \leq n-1, f^{*}\left(v_{(m-2) n}\right) \equiv 4 m n-4 n+4$.

The labels the vertices of $C_{n}^{(2)}, C_{n}^{(3)}, C_{n}^{(4)}, \ldots, C_{n}^{(m-1)}, C_{n}^{(m-3)}$ respectively are the same as in case (1).

The labels the vertices of $C_{n}^{(m)}$ are: $f^{*}\left(v_{(m-1) n+1}\right) \equiv 2 m n+2, f^{*}\left(v_{(m-1) n+2}\right) \equiv 2 m n+4, f^{*}\left(v_{(m-1) n+i}\right) \equiv$ $2 m n+2 i+2,3 \leq i \leq n$. Case (5): $n \equiv 8 \bmod 12$.

First, we label the edges of the paths $P_{m}^{(k)}, 1 \leq k \leq n$ begin with the edges of the path $P_{m}^{(1)}$ as the same in case (1).

Secondly, we label the edges of the circles $C_{n}^{(k)}, 1 \leq k \leq m$ begin with the edges of the inner most circle $C_{n}^{(1)}$, then the edges of outer circle $C_{n}^{(m)}$, then the edges of the circles $C_{n}^{(m-2)}, C_{n}^{(m-4)}, \ldots, C_{n}^{(3)}$.

Finally we label the edges of the circles $C_{m}^{(m-1)}, C_{m}^{(m-3)}, \ldots, C_{m}^{(2)}$

Label the edges of the circle $C_{n}^{(1)}$ as follows: $f\left(v_{1} v_{2}\right)=2 n(m-1)+2, f\left(v_{2} v_{3}\right)=2 n(m-1)+$ $6, f\left(v_{3} v_{4}\right)=2 n(m-1)+4, f\left(v_{4} v_{5}\right)=2 n(m-1)+8, f\left(v_{5} v_{6}\right)=2 n(m-1)+10, f\left(v_{6} v_{7}\right)=2 n(m-$ $1)+14, f\left(v_{7} v_{8}\right)=2 n(m-1)+12, f\left(v_{8} v_{9}\right)=2 n(m-1)+16, f\left(v_{9} v_{10}\right)=2 n(m-1)+18, f\left(v_{10} v_{11}\right)=$ 
$2 n(m-1)+22, f\left(v_{11} v_{12}\right)=2 n(m-1)+20, f\left(v_{12} v_{13}\right)=2 n(m-1)+24, f\left(v_{13} v_{14}\right)=2 n(m-1)+$ $26, \ldots, f\left(v_{n-9} v_{n-8}\right)=2 m n-20, f\left(v_{n-8} v_{n-7}\right)=2 m n-16, f\left(v_{n-7} v_{n-6}\right)=2 m n-14, f\left(v_{n-6} v_{n-5}\right)=$ $2 m n-10, f\left(v_{n-5} v_{n-4}\right)=2 m n-12, f\left(v_{n-4} v_{n-3}\right)=2 m n-8, f\left(v_{n-3} v_{n-2}\right)=2 m n-6, f\left(v_{n-2} v_{n-1}\right)=$ $2 m n-2, f\left(v_{n-1} v_{n}\right)=2 m n-4, f\left(v_{n} v_{1}\right)=2 m n+4$.

Label the edges of the circle $C_{n}^{(m)}$ as follows $f\left(v_{(m-1) n+1} v_{(m-1) n+2}\right)=2 m n+$ $2, f\left(v_{(m-1) n+2} v_{(m-1) n+3}\right)=2 m n+6, f\left(v_{(m-1) n+i} v_{(m-1) n+i+1}\right)=2 m n+2 i+2,3 \leq i \leq n-$ $1, f\left(v_{m n} v_{(m-1) n+1}\right)=2 m n$.

Label the edges of $C_{n}^{(m-2)}, C_{n}^{(m-4)}, \ldots, C_{n}^{(3)}$ and $C_{n}^{(m-1)}, C_{n}^{(m-3)}, C_{n}^{(m-5)}, \ldots, C_{n}^{(4)}, C_{n}^{(2)}$ as the same in case (1).

Thus we labels of corresponding vertices of the circle $C_{n}^{(1)} \bmod (4 m n-2 n)$ will be: $f^{*}\left(v_{1}\right) \equiv$ $8, f^{*}\left(v_{2}\right) \equiv 4 m n-4 n+12, f^{*}\left(v_{3}\right) \equiv 4 m n-4 n+16, f^{*}\left(v_{4}\right) \equiv 4 m n-4 n+20, f^{*}\left(v_{5}\right) \equiv 4 m n-4 n+28$, $f^{*}\left(v_{6}\right) \equiv 4 m n-4 n+36, f^{*}\left(v_{7}\right) \equiv 4 m n-4 n+40, f^{*}\left(v_{8}\right) \equiv 4 m n-4 n+44, f^{*}\left(v_{9}\right) \equiv 4 m n-4 n+52$, $f^{*}\left(v_{10}\right) \equiv 4 m n-4 n+60, f^{*}\left(v_{11}\right) \equiv 4 m n-4 n+64, f^{*}\left(v_{12}\right) \equiv 4 m n-4 n+68, f^{*}\left(v_{13}\right) \equiv 4 m n-4 n+76, \ldots$, $f^{*}\left(v_{n-8}\right) \equiv 4 n-52, f^{*}\left(v_{n-7}\right) \equiv 4 n-44, f^{*}\left(v_{n-6}\right) \equiv 4 n-36, f^{*}\left(v_{n-5}\right) \equiv 4 n-32, f^{*}\left(v_{n-4}\right) \equiv 4 n-28$, $f^{*}\left(v_{n-3}\right) \equiv 4 n-20, f^{*}\left(v_{n-2}\right) \equiv 4 n-12, f^{*}\left(v_{n-1}\right) \equiv 4 n-8, f^{*}\left(v_{n}\right) \equiv 4 n$.

The labels the vertices of the circle $C_{n}^{(m)}$ are: $f^{*}\left(v_{(m-1) n+1}\right) \equiv 2 m n-4 n+14, f^{*}\left(v_{(m-1) n+2}\right) \equiv$ $2 m n+8, f^{*}\left(v_{(m-1) n+i}\right) \equiv 2 m n+2 i+6,3 \leq i \leq n-1, f^{*}\left(v_{m n}\right) \equiv 2 m n+4$. The labels the vertices of $C_{n}^{(2)}, C_{n}^{(3)}, C_{n}^{(4)}, \ldots, C_{n}^{(m-2)}, C_{n}^{(m-1)}$ respectively are as the same in case (1).

Case (6): $n \equiv 10 \bmod 12$. First we label the edges of the paths $P_{m}^{(k)}, 1 \leq k \leq n$ begin with the edges of the path $P_{m}^{(1)}$ as the same as in case (1).

Second we label the edges of the circles $C_{n}^{(k)}, 1 \leq k \leq m$ begin with the edges of the inner most circle $C_{n}^{(1)}$, then the edges of outer circle $C_{n}^{(m)}$, then the edges of the circles $C_{n}^{(m-2)}, C_{n}^{(m-4)}, \ldots, C_{n}^{(3)}$.

Finally we label the edges of the circles $C_{m}^{(m-1)}, C_{m}^{(m-3)}, \ldots, C_{m}^{(2)}$.

Label the edges of the circle $C_{n}^{(1)}$ as follows: $f\left(v_{1} v_{2}\right)=2 n(m-1)+2, f\left(v_{2} v_{3}\right)=2 n(m-1)+$ $6, f\left(v_{3} v_{4}\right)=2 n(m-1)+4, f\left(v_{4} v_{5}\right)=2 n(m-1)+8, f\left(v_{5} v_{6}\right)=2 n(m-1)+10, f\left(v_{6} v_{7}\right)=2 n(m-$ $1)+14, f\left(v_{7} v_{8}\right)=2 n(m-1)+12, f\left(v_{8} v_{9}\right)=2 n(m-1)+16, f\left(v_{9} v_{10}\right)=2 n(m-1)+18, f\left(v_{10} v_{11}\right)=$ $2 n(m-1)+22, f\left(v_{11} v_{12}\right)=2 n(m-1)+20, f\left(v_{12} v_{13}\right)=2 n(m-1)+24, f\left(v_{13} v_{14}\right)=2 n(m-1)+$ $26, \ldots, f\left(v_{n-9} v_{n-8}\right)=2 m n-18, f\left(v_{n-8} v_{n-7}\right)=2 m n-14, f\left(v_{n-7} v_{n-6}\right)=2 m n-16, f\left(v_{n-6} v_{n-5}\right)=$ $2 m n-12, f\left(v_{n-5} v_{n-4}\right)=2 m n-10, f\left(v_{n-4} v_{n-3}\right)=2 m n-6, f\left(v_{n-3} v_{n-2}\right)=2 m n-8, f\left(v_{n-2} v_{n-1}\right)=$ $2 m n-4, f\left(v_{n-1} v_{n}\right)=2 m n-2, f\left(v_{n} v_{1}\right)=2 m n$.

Label the edges of the circle $C_{n}^{(2)}$ as follows: $f\left(v_{n+1} v_{n+2}\right)=4 n(m-1)+4, f\left(v_{n+2} v_{n+3}\right)=$ $4 n(m-1)+2, f\left(v_{n+i} v_{n+i+1}\right)=4 n(m-1)+2 i, 3 \leq i \leq n-2, f\left(v_{2 n-1} v_{2 n}\right)=2 n(2 m-1), f\left(v_{2 n} v_{2 n+1}\right)=$ $2 n(2 m-1)-2$.

Label the edges of $C_{n}^{(m)}, C_{n}^{(m-2)}, C_{n}^{(m-4)}, \ldots, C_{n}^{(3)}$ and $C_{n}^{(m-1)}, C_{n}^{(m-3)}, C_{n}^{(m-5)}, \ldots, C_{n}^{(4)}$ as in case (1).

Thus we have the labels of corresponding vertices $\bmod (4 m n-2 n)$ will be:

The labels the vertices of the circle $C_{n}^{(1)}$ are as follows: $f^{*}\left(v_{1}\right) \equiv 4, f^{*}\left(v_{2}\right) \equiv 4 m n-4 n+12, f^{*}\left(v_{3}\right) \equiv$ $4 m n-4 n+16, f^{*}\left(v_{4}\right) \equiv 4 m n-4 n+20, f^{*}\left(v_{5}\right) \equiv 4 m n-4 n+28, f^{*}\left(v_{6}\right) \equiv 4 m n-4 n+36, f^{*}\left(v_{7}\right) \equiv$ $4 m n-4 n+40, f^{*}\left(v_{8}\right) \equiv 4 m n-4 n+44, f^{*}\left(v_{9}\right) \equiv 4 m n-4 n+52, f^{*}\left(v_{10}\right) \equiv 4 m n-4 n+60, f^{*}\left(v_{11}\right) \equiv 4 m n-$ $4 n+64, f^{*}\left(v_{12}\right) \equiv 4 m n-4 n+68, f^{*}\left(v_{13}\right) \equiv 4 m n-4 n+76, f^{*}\left(v_{14}\right) \equiv 4 m n-4 n+84, \ldots, f^{*}\left(v_{n-8}\right) \equiv$ $4 n-48, f^{*}\left(v_{n-7}\right) \equiv 4 n-44, f^{*}\left(v_{n-6}\right) \equiv 4 n-40, f^{*}\left(v_{n-5}\right) \equiv 4 n-32, f^{*}\left(v_{n-4}\right) \equiv 4 n-24, f^{*}\left(v_{n-3}\right) \equiv$ $4 n-20, f^{*}\left(v_{n-2}\right) \equiv 4 n-16, f^{*}\left(v_{n-1}\right) \equiv 4 n-8, f^{*}\left(v_{n}\right) \equiv 4 n-2$.

The labels the vertices of the circle $C_{n}^{(2)}$ are as follows: $f^{*}\left(v_{n+1}\right) \equiv 6, f^{*}\left(v_{n+2}\right) \equiv 10, f^{*}\left(v_{n+3}\right) \equiv$ $12, f^{*}\left(v_{n+i}\right) \equiv 4 i+2,4 \leq i \leq n-2, f^{*}\left(v_{2 n-1}\right) \equiv 4 n, f^{*}\left(v_{2 n}\right) \equiv 4 n+2$. Label the vertices of $C_{n}^{(3)}, C_{n}^{(4)}, \ldots, C_{n}^{(m-2)}, C_{n}^{(m-1)}, C_{n}^{(m)}$ respectively are as the same as in case (1).

Illustration: The edge even graceful labeling of the cylinder grid graphs $C_{9,2}, C_{9,4}, C_{7,10}, C_{7,12}, C_{7,14} C_{7,16} C_{7,18}$ and $C_{7,20}$ are shown in Figure 12. 


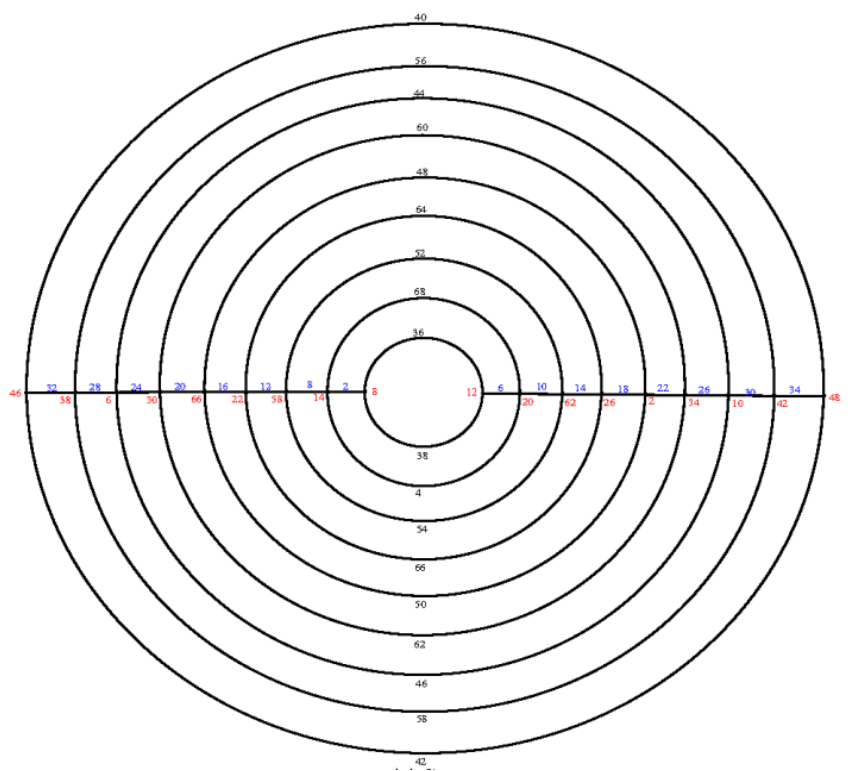

(a) $C_{9,2}$

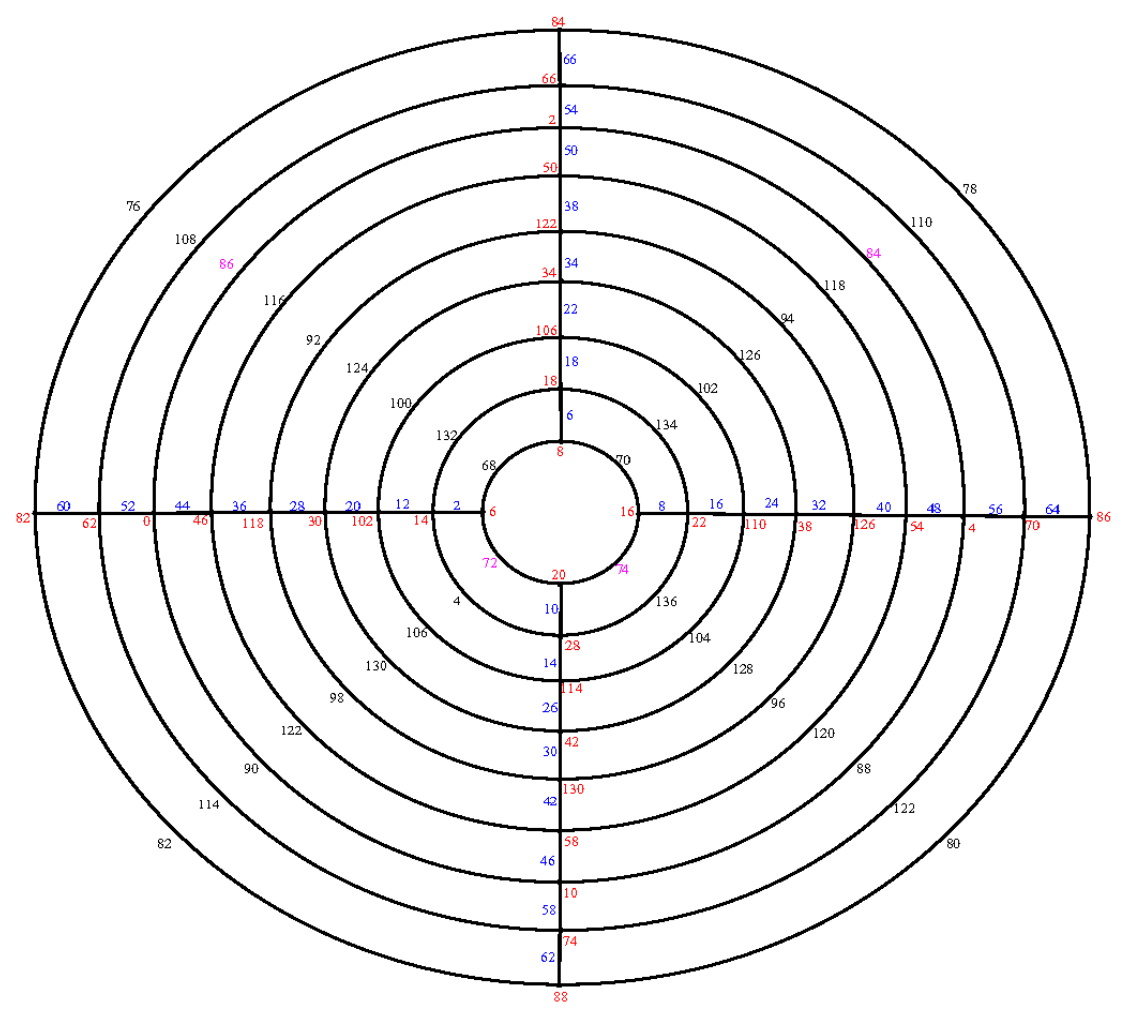

(b) $C_{9,4}$

Figure 12. Cont. 


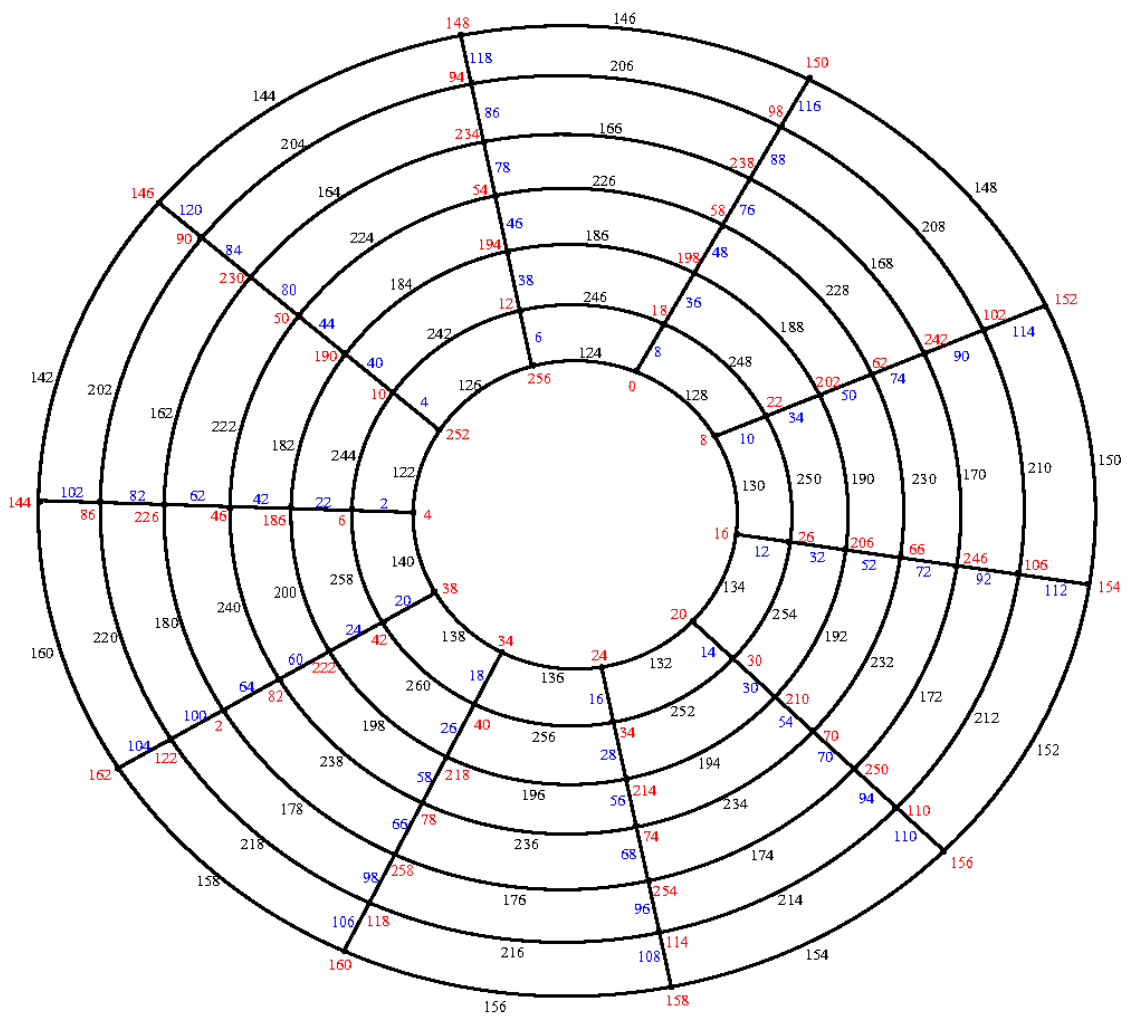

(c) $C_{7,10}$

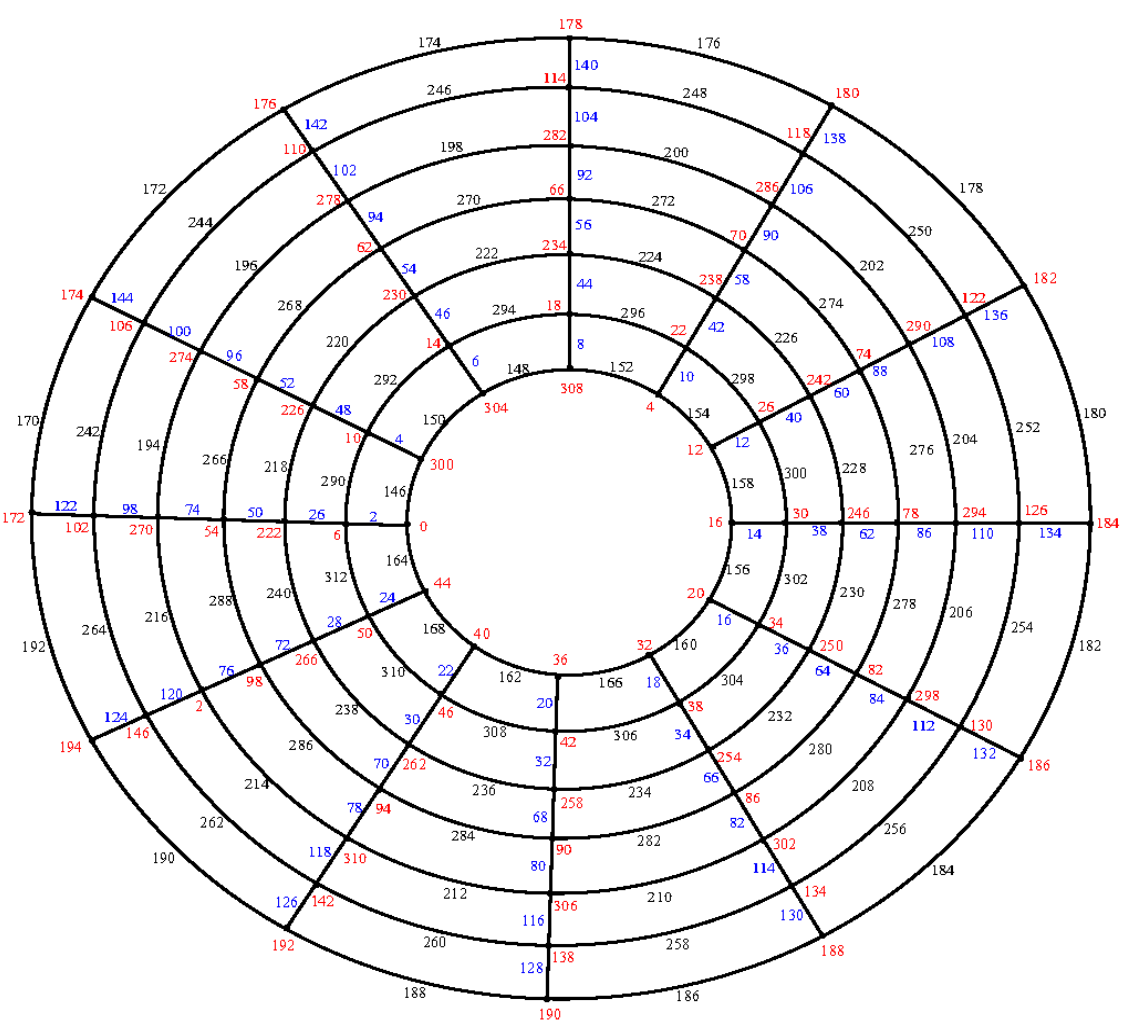

(d) $C_{7,12}$

Figure 12. Cont. 


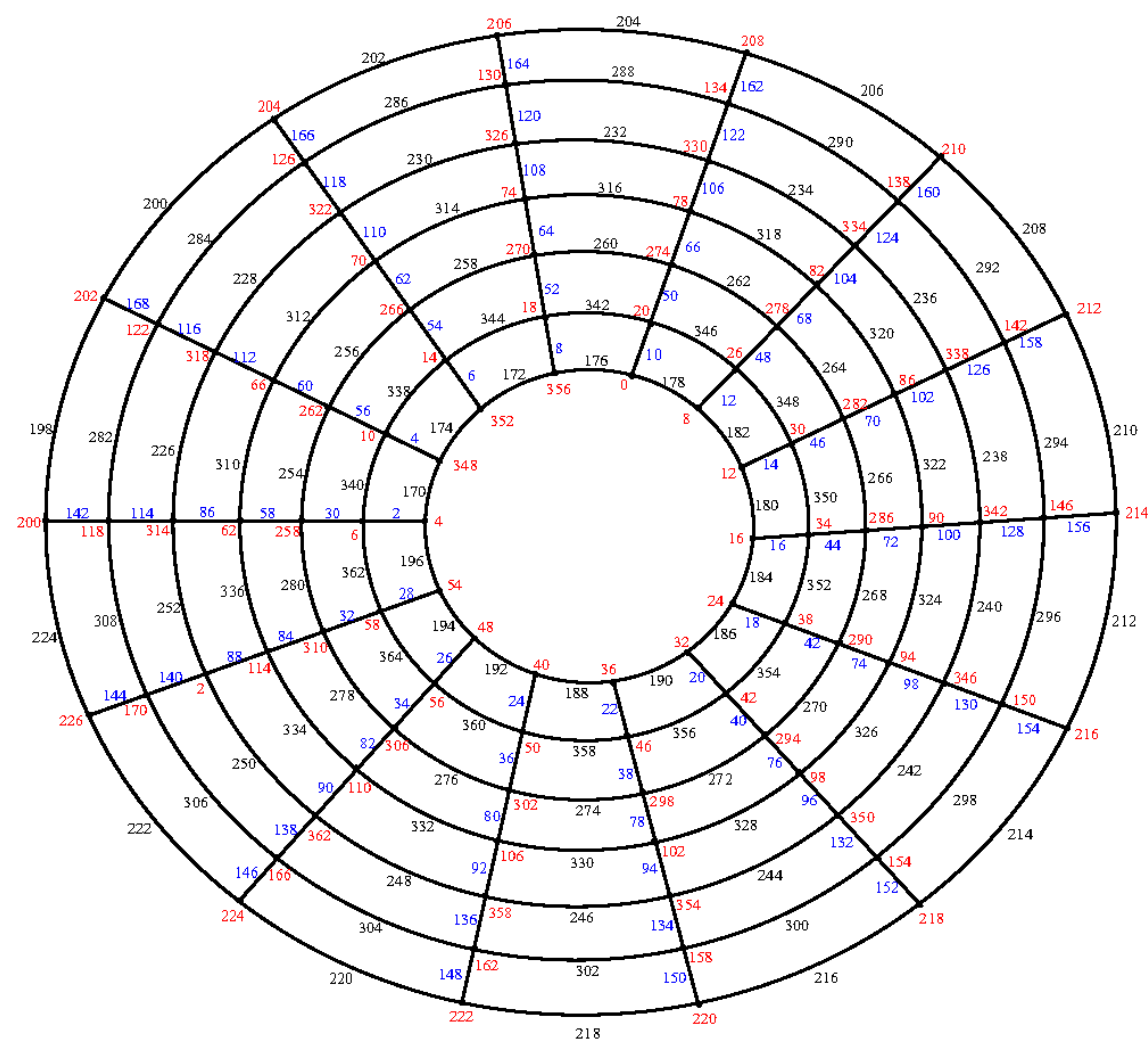

(e) $C_{7,14}$

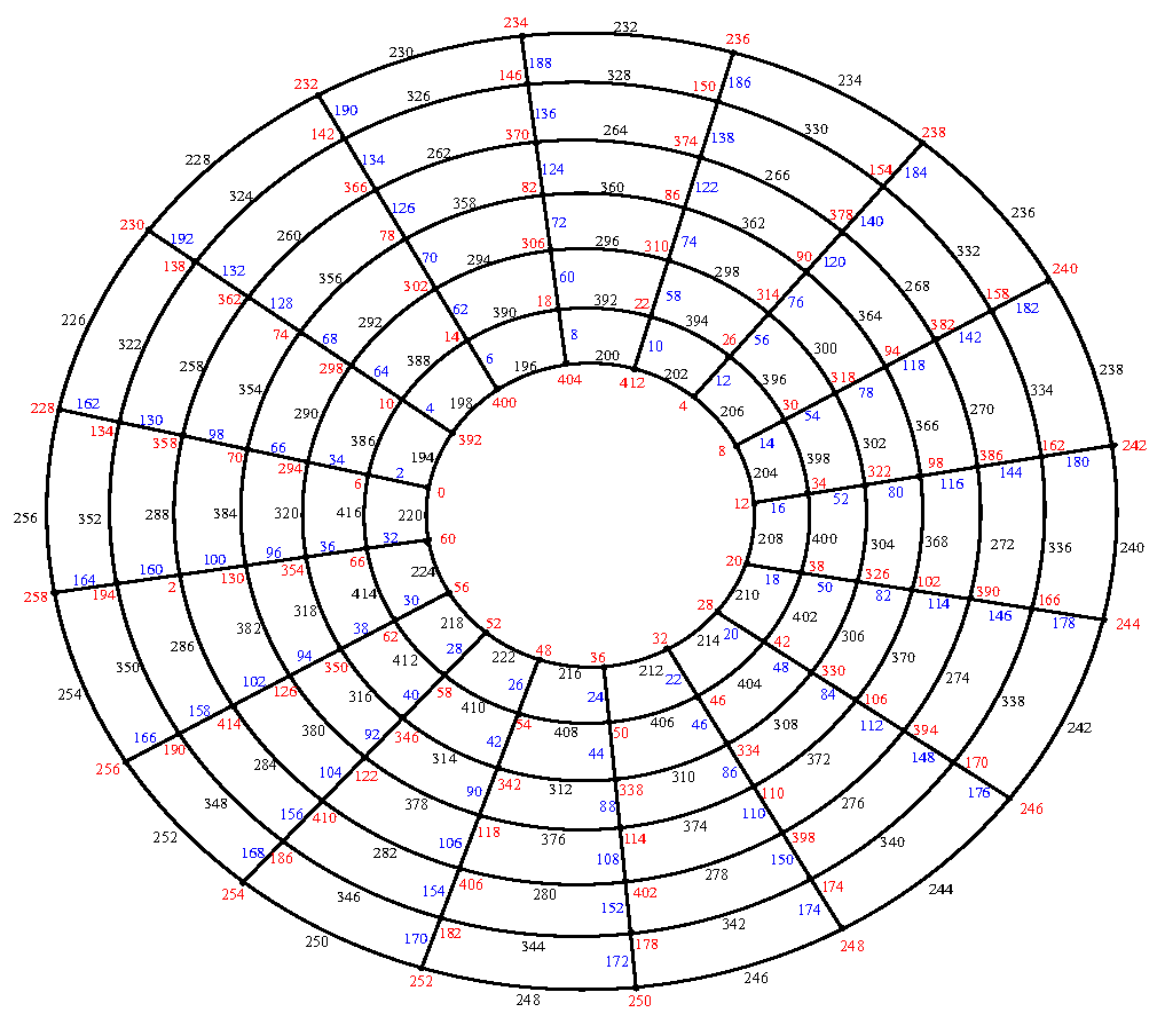

(f) $C_{7,16}$

Figure 12. Cont. 


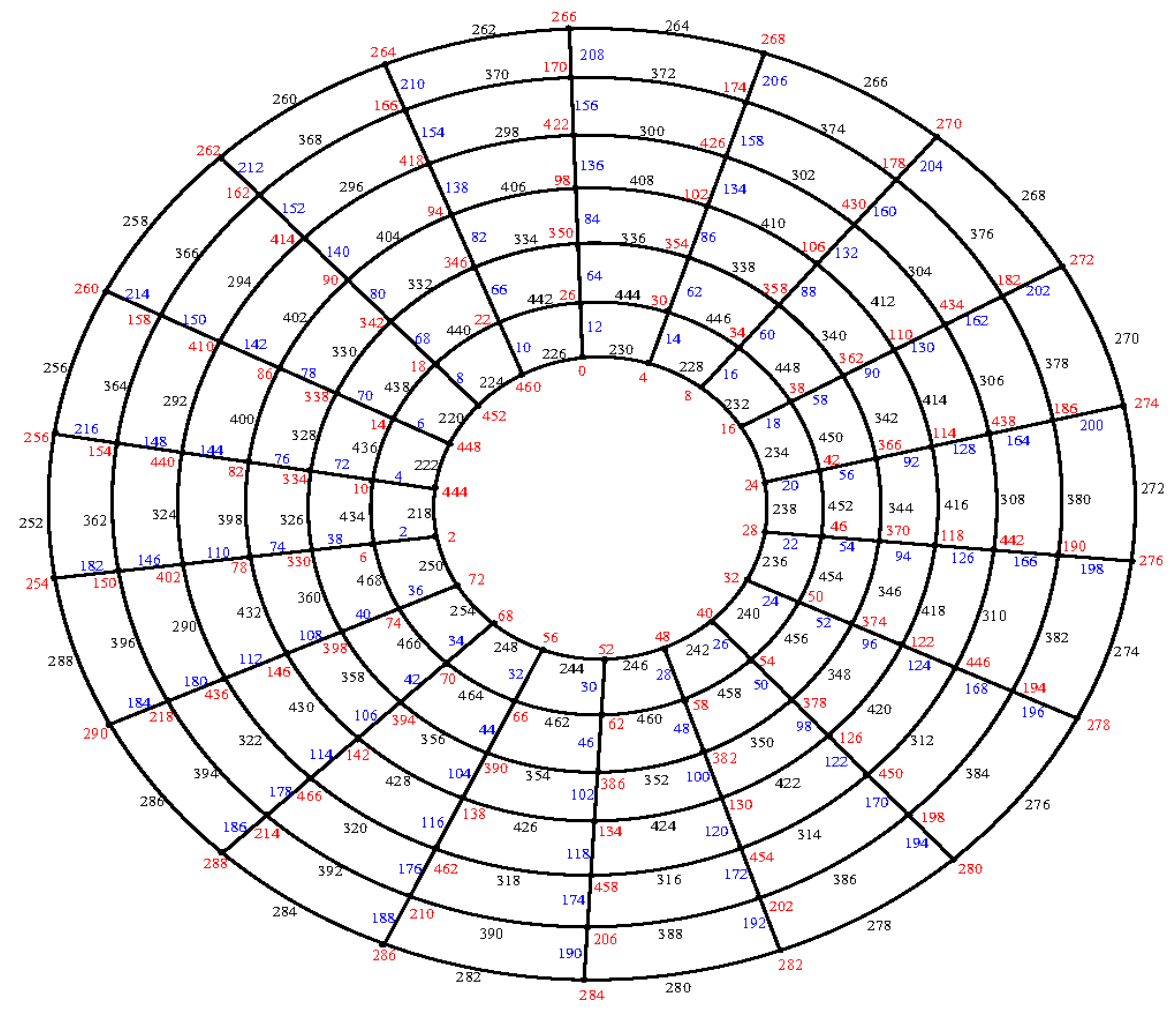

(g) $C_{7,18}$

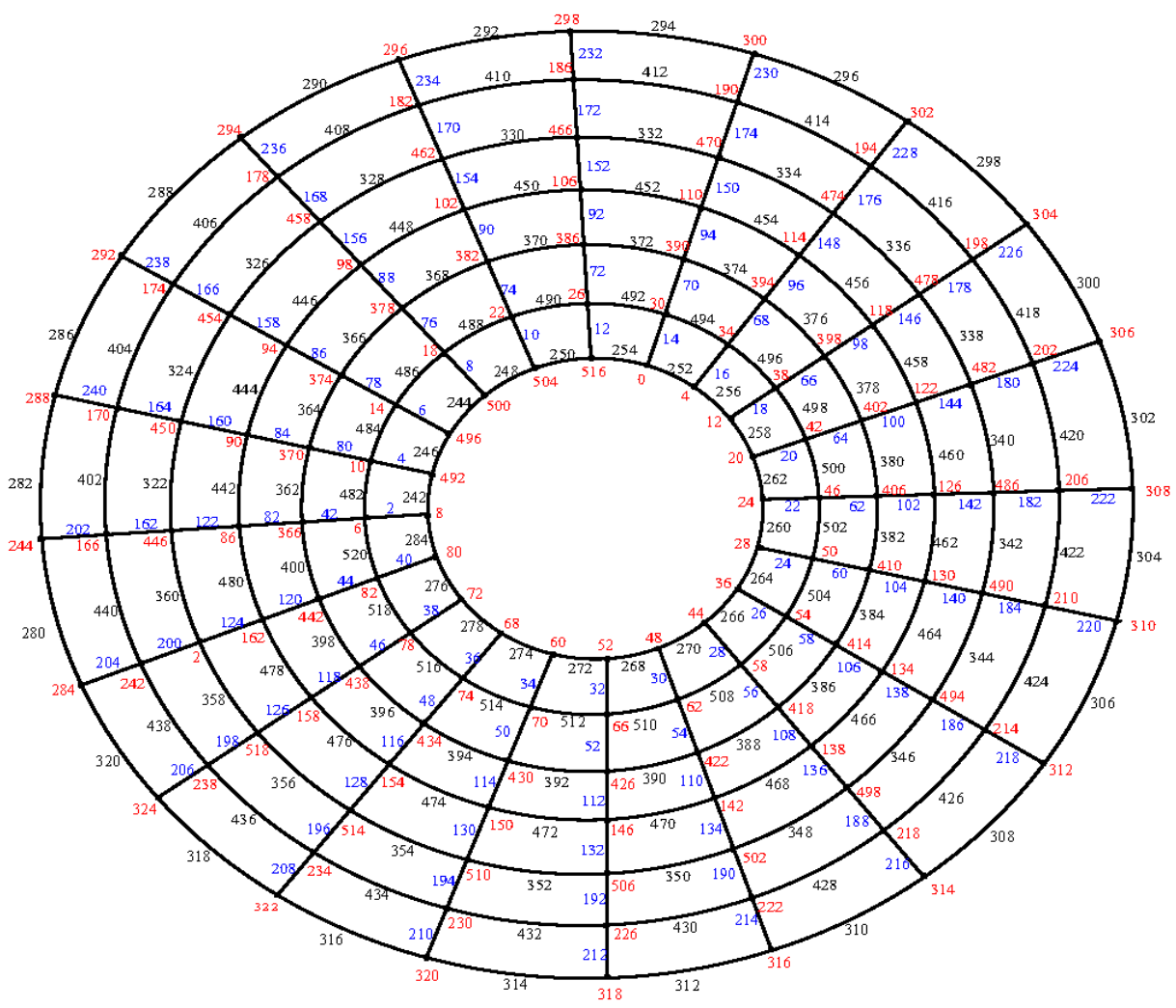

(h) $C_{7,20}$

Figure 12. An e.e.g., l. of the cylinder grid graphs $C_{9,2}, C_{9,4}, C_{7,10}, C_{7,12}, C_{7,14}, C_{7,16}, C_{7,18}$, and $C_{7,20}$. 
Theorem 4. If $m$ is an odd positive integer greater than 3 and $n$ is an odd positive integer, $n \geq 3$, then the cylinder grid graph $C_{m, n}$, is an edge even graceful graph.

Proof. Using standard notation $p=\left|V\left(C_{m, n}\right)\right|=m n, q=\left|E\left(C_{m, n}\right)\right|=2 m n-n, r=\max (p, q)=2 m n-n$, and $f: E\left(C_{m, n}\right) \rightarrow\{2,4,6, \ldots, 4 m n-2 n-2\}$.

Let the cylinder grid graph $C_{m, n}$ be as in Figure 9. There are six cases:

Case (1): $n \equiv 1 \bmod 12$.

First, we label the edges of the paths $P_{m}^{(k)}, 1 \leq k \leq n$ beginning with the edges of the path $P_{m}^{(1)}$ as follows: Move clockwise to label the edges $v_{1} v_{n+1}, v_{2} v_{n+2}, v_{3} v_{n+3}, \ldots, v_{n-1} v_{2 n-1}, v_{n} v_{2 n}$ by $2,4,6, \ldots, 2 n-$ $2,2 n$, then move anticlockwise to label the edges $v_{n+1} v_{n+2}, v_{2 n} v_{3 n}, v_{2 n-1} v_{3 n-1}, \ldots, v_{n+3} v_{2 n+3}, v_{n+2} v_{2 n+2}$ by $2 n+2,2 n+4,2 n+6, \ldots, 4 n-2,4 n$, then move clockwise to label the edges $v_{2 n+1} v_{3 n+1}, v_{2 n+2} v_{3 n+2}, v_{2 n+3} v_{3 n+3}, \ldots, v_{3 n-1} v_{4 n-1}, v_{3 n} v_{4 n}$ by $4 n+2,4 n+4,4 n+6, \ldots, 6 n-2,6 n$, and so on.

Finally, move anticlockwise to label the edges $v_{(m-2) n+1} v_{(m-1) n+1}, v_{(m-1) n} v_{m n}, v_{(m-1) n-1} v_{m n-1}, \ldots$, $v_{(m-2) n+3} v_{(m-1) n+3}, v_{(m-2) n+2} v_{m(n-1)+2}$ by $2 n(m-2)+2,2 n(m-2)+4,2 n(m-2)+6, \ldots, 2 n(m-1)-$ $2,2 n(m-1)$.

Second, we label the edges of the circles $C_{n}^{(k)}, 1 \leq k \leq m$ beginning with the edges of the innermost circle $C_{n}^{(1)}$, then the edges of outer circle $C_{n}^{(m)}$, and then the edges of the circles $C_{n}^{(m-2)}, C_{n}^{(m-4)}, \ldots, C_{n}^{(3)}$.

Finally, we label the edges of the circles $C_{m}^{(m-1)}, C_{m}^{(m-3)}, \ldots, C_{m}^{(2)}$.

Label the edges of $C_{n}^{(1)}$ as follows: $f\left(v_{1} v_{2}\right)=2 n(m-1)+2, f\left(v_{2} v_{3}\right)=2 n(m-1)+4, f\left(v_{3} v_{4}\right)=$ $2 n(m-1)+8, f\left(v_{4} v_{5}\right)=2 n(m-1)+6, f\left(v_{5} v_{6}\right)=2 n(m-1)+10, f\left(v_{6} v_{7}\right)=2 n(m-1)+$ $12, f\left(v_{7} v_{8}\right)=2 n(m-1)+16, f\left(v_{8} v_{9}\right)=2 n(m-1)+14, f\left(v_{9} v_{10}\right)=2 n(m-1)+18, f\left(v_{10} v_{11}\right)=$ $2 n(m-1)+20, f\left(v_{11} v_{12}\right)=2 n(m-1)+24, f\left(v_{12} v_{13}\right)=2 n(m-1)+22, f\left(v_{13} v_{14}\right)=2 n(m-1)+26$, $f\left(v_{14} v_{15}\right)=2 n(m-1)+28, \ldots, f\left(v_{n-7} v_{n-6}\right)=2 m n-14, f\left(v_{n-6} v_{n-5}\right)=2 m n-10, f\left(v_{n-5} v_{n-4}\right)=$ $2 m n-12, f\left(v_{n-4} v_{n-3}\right)=2 m n-8, f\left(v_{n-3} v_{n-2}\right)=2 m n-6, f\left(v_{n-2} v_{n-1}\right)=2 m n-2, f\left(v_{n-1} v_{n}\right)=$ $2 m n-4, f\left(v_{n} v_{1}\right)=2 m n$.

Then, label the edges of $C_{n}^{(m)}, C_{n}^{(m-2)}, C_{n}^{(m-4)}, \ldots, C_{n}^{(3)}$ and $C_{n}^{(m-1)}, C_{n}^{(m-3)}, C_{n}^{(m-5)}, \ldots, C_{n}^{(4)}, C_{n}^{(2)}$ as follows:

Label the edges of the circle $C_{n}^{(m)}$ as follows: $f\left(v_{(m-1) n+i} v_{(m-1) n+i+1}\right)=2 m n+$ $2 i, f\left(v_{m n} v_{(m-1) n+1}\right)=2 n(m+1), 1 \leq i \leq n-1$.

Label the edges of the circle $C_{n}^{(m-2)}$ as follows: $f\left(v_{(m-3) n+i} v_{(m-3) n+i+1}\right)=2 n(m+1)+$ $2 i, f\left(v_{(m-2) n} v_{(m-3) n+1}\right)=2 n(m+2), 1 \leq i \leq n-1$.

Label the edges of the circle $C_{n}^{(m-4)}$ as follows: $f\left(v_{(m-5) n+i} v_{(m-5) n+i+1}\right)=2 n(m+2)+$ $2 i, f\left(v_{(m-4) n} v_{(m-5) n+1}\right)=2 n(m+3), 1 \leq i \leq n-1$, and so on.

Label the edges of the circle $C_{n}^{(3)}$ as follows: $f\left(v_{2 n+i} v_{2 n+i+1}\right)=3 n(m-1)+2 i, f\left(v_{3 n} v_{2 n+1}\right)=$ $n(3 m-1), 1 \leq i \leq n-1$,

Label the edges of the circle $C_{n}^{(m-1)}$ as follows: $f\left(v_{(m-2) n+i} v_{(m-2) n+i+1}\right)=n(3 m-1)+$ $2 i, f\left(v_{(m-1) n} v_{(m-1) n+1}\right)=n(3 m+1), 1 \leq i \leq n-1$,

Label the edges of the circle $C_{n}^{(m-3)}$ as follows: $f\left(v_{(m-4) n+i} v_{(m-4) n+i+1}\right)=n(3 m+2)+$ $2 i, f\left(v_{(m-3) n} v_{(m-4) n+1}\right)=3 n(m+1), 1 \leq i \leq n-1, \ldots$, and so on.

Label the edges of the circle $C_{n}^{(4)}$ as follows: $f\left(v_{3 n+i} v_{3 n+i+1}\right)=2 n(2 m-3)+2 i, f\left(v_{4 n} v_{3 n+1}\right)=$ $4 n(m-1), 1 \leq i \leq n-1$,

Label the edges of $C_{n}^{(2)}$ as follows: $f\left(v_{n+i} v_{n+i+1}\right)=4 n(m-1)+2 i, f\left(v_{2 n} v_{2 n+1}\right)=2 n(m-1), 1 \leq$ $i \leq n-1$,

Thus, the labels of corresponding vertices of the $\operatorname{circle} C_{n}^{(1)} \bmod (4 m n-2 n)$ will be: $f^{*}\left(v_{1}\right) \equiv 4, f^{*}\left(v_{2}\right) \equiv$ $4 m n-4 n+10, f^{*}\left(v_{3}\right) \equiv 4 m n-4 n+18, f^{*}\left(v_{4}\right) \equiv 4 m n-4 n+22, f^{*}\left(v_{5}\right) \equiv 4 m n-4 n+26, f^{*}\left(v_{6}\right) \equiv 4 m n-$ $4 n+34, f^{*}\left(v_{7}\right) \equiv 4 m n-4 n+42, f^{*}\left(v_{8}\right) \equiv 4 m n-4 n+46, f^{*}\left(v_{9}\right) \equiv 4 m n-4 n+50, f^{*}\left(v_{10}\right) \equiv 4 m n-$ 
$4 n+58, f^{*}\left(v_{11}\right) \equiv 4 m n-4 n+66, f^{*}\left(v_{12}\right) \equiv 4 m n-4 n+70, f^{*}\left(v_{13}\right) \equiv 4 m n-4 n+74, f^{*}\left(v_{14}\right) \equiv 4 m n-$ $4 n+82, \ldots, f^{*}\left(v_{n-7}\right) \equiv 4 n-44, f^{*}\left(v_{n-6}\right) \equiv 4 n-36, f^{*}\left(v_{n-5}\right) \equiv 4 n-32, f^{*}\left(v_{n-4}\right) \equiv 4 n-28, f^{*}\left(v_{n-3}\right) \equiv$ $4 n-20, f^{*}\left(v_{n-2}\right) \equiv 4 n-12, f^{*}\left(v_{n-1}\right) \equiv 4 n-8, f^{*}\left(v_{n}\right) \equiv 4 n-4$.

The labels of the vertices of $C_{n}^{(2)}, C_{n}^{(3)}, C_{n}^{(4)}, \ldots, C_{n}^{(m-2)}, C_{n}^{(m-1)}, C_{n}^{(m)}$, respectively, are as follows: $f^{*}\left(v_{n+i}\right) \equiv 4 i+2 ; f^{*}\left(v_{2 n+i}\right) \equiv 2 m n+4 n+4 i+2 ; f^{*}\left(v_{3 n+i}\right) \equiv 4 n+4 i+2 ; \ldots ; f^{*}\left(v_{(m-3) n+i}\right) \equiv 4 m n-$ $6 n+4 i+2 ; f^{*}\left(v_{(m-2) n+i}\right) \equiv 2 m n-6 n+4 i+2 ; f^{*}\left(v_{(m-1) n+i}\right) \equiv 2 m n+2 i+2,1 \leq i \leq n$.

Case (2): $n \equiv 3 \bmod 12$.

First, we label the edges of the paths $P_{m}^{(k)}, 1 \leq k \leq n$ beginning with the edges of the path $P_{m}^{(1)}$ as the same in case (1).

Second, we label the edges of the circles $C_{n}^{(k)}, 1 \leq k \leq m$ beginning with the edges of the innermost circle $C_{n}^{(1)}$, then the edges of outer circle $C_{n}^{(m)}$, and then the edges of the circles $C_{n}^{(m-2)}, C_{n}^{(m-4)}, \ldots, C_{n}^{(3)}$.

Finally, we label the edges of the circles $C_{m}^{(m-1)}, C_{m}^{(m-3)}, \ldots, C_{m}^{(2)}$.

Label the edges of the circle $C_{n}^{(1)}$ as follows: $f\left(v_{1} v_{2}\right)=2 n(m-1)+2, f\left(v_{2} v_{3}\right)=2 n(m-1)+$ $4, f\left(v_{3} v_{4}\right)=2 n(m-1)+8, f\left(v_{4} v_{5}\right)=2 n(m-1)+6, f\left(v_{5} v_{6}\right)=2 n(m-1)+10, f\left(v_{6} v_{7}\right)=2 n(m-$ 1) $+12, f\left(v_{7} v_{8}\right)=2 n(m-1)+16, f\left(v_{8} v_{9}\right)=2 n(m-1)+14, f\left(v_{9} v_{10}\right)=2 n(m-1)+18, f\left(v_{10} v_{11}\right)=$ $2 n(m-1)+20, f\left(v_{11} v_{12}\right)=2 n(m-1)+24, f\left(v_{12} v_{13}\right)=2 n(m-1)+22, f\left(v_{13} v_{14}\right)=2 n(m-1)+$ $26, \ldots, f\left(v_{n-9} v_{n-8}\right)=2 m n-18, f\left(v_{n-8} v_{n-7}\right)=2 m n-14, f\left(v_{n-7} v_{n-6}\right)=2 m n-16, f\left(v_{n-6} v_{n-5}\right)=$ $2 m n-12, f\left(v_{n-5} v_{n-4}\right)=2 m n-10, f\left(v_{n-4} v_{n-3}\right)=2 m n-6, f\left(v_{n-3} v_{n-2}\right)=2 m n-8, f\left(v_{n-2} v_{n-1}\right)=$ $2 m n, f\left(v_{n-1} v_{n}\right)=2 m n-2, f\left(v_{n} v_{1}\right)=2 m n-4$.

Label the edges of the circle $C_{n}^{(2)}$ as follows: $f\left(v_{n+i} v_{n+i+1}\right)=4 n(m-1)+2 i, 1 \leq i \leq n-$ $9, f\left(v_{2 n-9} v_{2 n-8}\right)=2 n(2 m-1)-18, f\left(v_{2 n-8} v_{2 n-7}\right)=2 n(2 m-1)-14, f\left(v_{2 n-7} v_{2 n-6}\right)=2 n(2 m-1)-16$, $f\left(v_{2 n-6} v_{2 n-5}\right)=2 n(2 m-1)-10, f\left(v_{2 n-5} v_{2 n-4}\right)=2 n(2 m-1)-12, f\left(v_{2 n-4} v_{2 n-3}\right)=2 n(2 m-1)-6$, $f\left(v_{2 n-3} v_{2 n-2}\right)=2 n(2 m-1)-8, f\left(v_{2 n-2} v_{2 n-1}\right)=2 n(2 m-1)-4, f\left(v_{2 n-1} v_{2 n}\right)=2 n(2 m-1)-$ $2, f\left(v_{2 n} v_{n+1}\right)=2 n(2 m-1)$.

Label the edges of $C_{n}^{(m)}, C_{n}^{(m-2)}, C_{n}^{(m-4)}, \ldots, C_{n}^{(3)}$ and $C_{n}^{(m-1)}, C_{n}^{(m-3)}, C_{n}^{(m-5)}, \ldots, C_{n}^{(4)}$ as in case (1).

Thus, the labels of corresponding vertices $\bmod (4 m n-2 n)$ will be:

The labels of the vertices of $C_{n}^{(1)}$ are as follows: $f^{*}\left(v_{1}\right) \equiv 0, f^{*}\left(v_{2}\right) \equiv 4 m n-4 n+10, f^{*}\left(v_{3}\right) \equiv$ $4 m n-4 n+18, f^{*}\left(v_{4}\right) \equiv 4 m n-4 n+22, f^{*}\left(v_{5}\right) \equiv 4 m n-4 n+26, f^{*}\left(v_{6}\right) \equiv 4 m n-4 n+34, f^{*}\left(v_{7}\right) \equiv$ $4 m n-4 n+42, f^{*}\left(v_{8}\right) \equiv 4 m n-4 n+46, f^{*}\left(v_{9}\right) \equiv 4 m n-4 n+50, f^{*}\left(v_{10}\right) \equiv 4 m n-4 n+58, f^{*}\left(v_{11}\right) \equiv 4 m n-$ $4 n+66, f^{*}\left(v_{12}\right) \equiv 4 m n-4 n+70, f^{*}\left(v_{13}\right) \equiv 4 m n-4 n+74, f^{*}\left(v_{14}\right) \equiv 4 m n-4 n+82, \ldots, f^{*}\left(v_{n-8}\right) \equiv$ $4 n-48, f^{*}\left(v_{n-7}\right) \equiv 4 n-44, f^{*}\left(v_{n-6}\right) \equiv 4 n-40, f^{*}\left(v_{n-5}\right) \equiv 4 n-32, f^{*}\left(v_{n-4}\right) \equiv 4 n-24, f^{*}\left(v_{n-3}\right) \equiv$ $4 n-20, f^{*}\left(v_{n-2}\right) \equiv 4 n-12, f^{*}\left(v_{n-1}\right) \equiv 4 n-4, f^{*}\left(v_{n}\right) \equiv 4 n-6$.

The labels of the vertices of the circle $C_{n}^{(2)}$ are as follows: $f^{*}\left(v_{n+i}\right) \equiv 4 i+2,1 \leq i \leq n-$ $9, f^{*}\left(v_{2 n-8}\right) \equiv 4 n-28, f^{*}\left(v_{2 n-7}\right) \equiv 4 n-26, f^{*}\left(v_{2 n-6}\right) \equiv 4 n-22, f^{*}\left(v_{2 n-5}\right) \equiv 4 n-18, f^{*}\left(v_{2 n-4}\right) \equiv 4 n-14$, $f^{*}\left(v_{2 n-3}\right) \equiv 4 n-10, f^{*}\left(v_{2 n-2}\right) \equiv 4 n-8, f^{*}\left(v_{2 n-1}\right) \equiv 4 n-2, f^{*}\left(v_{2 n}\right) \equiv 4 n+2$.

The labels of the vertices of $C_{n}^{(3)}, C_{n}^{(4)}, \ldots, C_{n}^{(m-2)}, C_{n}^{(m-1)}, C_{n}^{(m)}$, respectively, are the same as in case (1).

Remark 4. In case $n=3$ and $m$ is odd, $m \geq 3$.

Let the label of edges of the cylinder grid graph $C_{m, 3}$ be as in Figure 13.

Thus, the labels of corresponding vertices $\bmod (12 m-6)$ are as follows:

The labels of the vertices of the circle $C_{3}^{(1)}$ are $f^{*}\left(v_{1}\right) \equiv 8, f^{*}\left(v_{2}\right) \equiv 12, f^{*}\left(v_{3}\right) \equiv 16$.

The labels of the vertices of the circle $C_{3}^{(3)}$ are $f^{*}\left(v_{3 m-2}\right) \equiv 6 m+10, f^{*}\left(v_{3 m-1}\right) \equiv 6 m+12, f^{*}\left(v_{3 m}\right) \equiv$ $6 m+14$.

The labels of the vertices of the circles $C_{3}^{(2)}, C_{3}^{(4)}, \ldots, C_{3}^{(m-1)}$ are $f^{*}\left(v_{3 k+i}\right) \equiv 4 i+6 k+4,1 \leq i \leq 3,1 \leq$ $k \leq m-2, k$ is odd.

The labels of the vertices of the circles $C_{3}^{(3)}, C_{3}^{(5)}, \ldots, C_{3}^{(m-2)}$ are $f^{*}\left(v_{3 k+i}\right) \equiv 6 m+4 i+6 k+10,1 \leq i \leq$ $3,2 \leq k \leq m-3, k$ is even. 


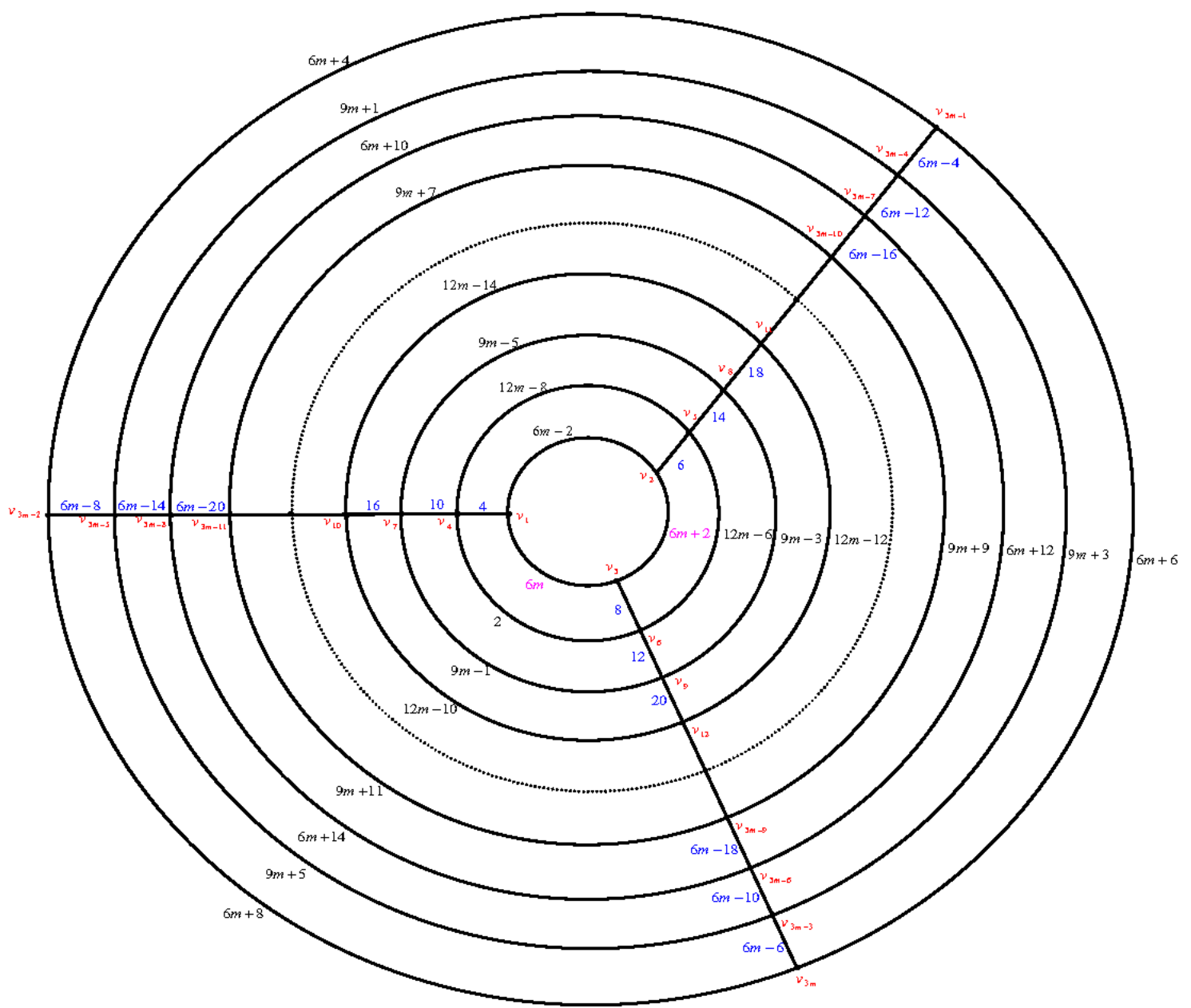

Figure 13. The cylinder grid graph $C_{m, 3}, m$ is odd, $m \geq 3$.

Case (3): $n \equiv 5 \bmod 12$.

First, we label the edges of the paths $P_{m}^{(k)}, 1 \leq k \leq n$ beginning with the edges of the path $P_{m}^{(1)}$ the same as in case (1).

Second, we label the edges of the circles $C_{n}^{(k)}, 1 \leq k \leq m$ beginning with the edges of the innermost circle $C_{n}^{(1)}$, then the edges of outer circle $C_{n}^{(m)}$, and then the edges of the circles $C_{n}^{(m-2)}, C_{n}^{(m-4)}, \ldots, C_{n}^{(3)}$.

Finally, we label the edges of the circles $C_{m}^{(m-1)}, C_{m}^{(m-3)}, \ldots, C_{m}^{(2)}$.

Label the edges of the circle $C_{n}^{(1)}$ as follows: $f\left(v_{1} v_{2}\right)=2 n(m-1)+2, f\left(v_{2} v_{3}\right)=2 n(m-1)+$ $4, f\left(v_{3} v_{4}\right)=2 n(m-1)+8, f\left(v_{4} v_{5}\right)=2 n(m-1)+6, f\left(v_{5} v_{6}\right)=2 n(m-1)+10, f\left(v_{6} v_{7}\right)=2 n(m-$ $1)+12, f\left(v_{7} v_{8}\right)=2 n(m-1)+16, f\left(v_{8} v_{9}\right)=2 n(m-1)+14, f\left(v_{9} v_{10}\right)=2 n(m-1)+18, f\left(v_{10} v_{11}\right)=$ $2 n(m-1)+20, f\left(v_{11} v_{12}\right)=2 n(m-1)+24, f\left(v_{12} v_{13}\right)=2 n(m-1)+22, f\left(v_{13} v_{14}\right)=2 n(m-1)+$ $26, \ldots, f\left(v_{n-8} v_{n-7}\right)=2 m n-16, f\left(v_{n-7} v_{n-6}\right)=2 m n-14, f\left(v_{n-6} v_{n-5}\right)=2 m n-10, f\left(v_{n-5} v_{n-4}\right)=$ $2 m n-12, f\left(v_{n-4} v_{n-3}\right)=2 m n-8, f\left(v_{n-3} v_{n-2}\right)=2 m n-6, f\left(v_{n-2} v_{n-1}\right)=2 m n-2, f\left(v_{n-1} v_{n}\right)=2 m n-4$, $f\left(v_{n} v_{1}\right)=2 m n$. Label the edges of $C_{n}^{(m)}, C_{n}^{(m-2)}, C_{n}^{(m-4)}, \ldots, C_{n}^{(3)}$ and $C_{n}^{(m-1)}, C_{n}^{(m-3)}, C_{n}^{(m-5)}, \ldots, C_{n}^{(4)}, C_{n}^{(2)}$ as in case (1).

Thus, the labels of corresponding vertices of the circle $C_{n}^{(1)} \bmod (4 m n-2 n)$ will be: $f^{*}\left(v_{1}\right) \equiv$ $4, f^{*}\left(v_{2}\right) \equiv 4 m n-4 n+10, f^{*}\left(v_{3}\right) \equiv 4 m n-4 n+18, f^{*}\left(v_{4}\right) \equiv 4 m n-4 n+22, f^{*}\left(v_{5}\right) \equiv 4 m n-4 n+26$, $f^{*}\left(v_{6}\right) \equiv 4 m n-4 n+34, f^{*}\left(v_{7}\right) \equiv 4 m n-4 n+42, f^{*}\left(v_{8}\right) \equiv 4 m n-4 n+46, f^{*}\left(v_{9}\right) \equiv 4 m n-4 n+50$, $f^{*}\left(v_{10}\right) \equiv 4 m n-4 n+58, f^{*}\left(v_{11}\right) \equiv 4 m n-4 n+66, f^{*}\left(v_{12}\right) \equiv 4 m n-4 n+70, f^{*}\left(v_{13}\right) \equiv 4 m n-4 n+74$, $f^{*}\left(v_{14}\right) \equiv 4 m n-4 n+82, \ldots, f^{*}\left(v_{n-7}\right) \equiv 4 n-44, f^{*}\left(v_{n-6}\right) \equiv 4 n-36, f^{*}\left(v_{n-5}\right) \equiv 4 n-32, f^{*}\left(v_{n-4}\right) \equiv$ $4 n-28, f^{*}\left(v_{n-3}\right) \equiv 4 n-20, f^{*}\left(v_{n-2}\right) \equiv 4 n-12, f^{*}\left(v_{n-1}\right) \equiv 4 n-8, f^{*}\left(v_{n}\right) \equiv 4 n-4$. 
The labels of the vertices of $C_{n}^{(2)}, C_{n}^{(3)}, C_{n}^{(4)}, \ldots, C_{n}^{(m-2)}, C_{n}^{(m-1)}, C_{n}^{(m)}$, respectively, are the same as in case (1).

Case (4): $n \equiv 7 \bmod 12$.

First, we label the edges of the paths $P_{m}^{(k)}, 1 \leq k \leq n$ beginning with the edges of the path $P_{m}^{(1)}$ the same as in case (1).

Second, we label the edges of the circles $C_{n}^{(k)}, 1 \leq k \leq m$ beginning with the edges of the innermost circle $C_{n}^{(1)}$, then the edges of outer circle $C_{n}^{(m)}$, and then the edges of the circles $C_{n}^{(m-2)}, C_{n}^{(m-4)}, \ldots, C_{n}^{(3)}$.

Finally, we label the edges of the circles $C_{m}^{(m-1)}, C_{m}^{(m-3)}, \ldots, C_{m}^{(2)}$.

Label the edges of the circle $C_{n}^{(1)}$ as follows: $f\left(v_{1} v_{2}\right)=2 n(m-1)+2, f\left(v_{2} v_{3}\right)=2 n(m-1)+$ $4, f\left(v_{3} v_{4}\right)=2 n(m-1)+8, f\left(v_{4} v_{5}\right)=2 n(m-1)+6, f\left(v_{5} v_{6}\right)=2 n(m-1)+10, f\left(v_{6} v_{7}\right)=2 n(m-$ $1)+12, f\left(v_{7} v_{8}\right)=2 n(m-1)+16, f\left(v_{8} v_{9}\right)=2 n(m-1)+14, f\left(v_{9} v_{10}\right)=2 n(m-1)+18, f\left(v_{10} v_{11}\right)=$ $2 n(m-1)+20, f\left(v_{11} v_{12}\right)=2 n(m-1)+24, f\left(v_{12} v_{13}\right)=2 n(m-1)+22, f\left(v_{13} v_{14}\right)=2 n(m-1)+$ $26, \ldots, f\left(v_{n-10} v_{n-9}\right)=2 m n-20, f\left(v_{n-9} v_{n-8}\right)=2 m n-18, f\left(v_{n-8} v_{n-7}\right)=2 m n-14, f\left(v_{n-7} v_{n-6}\right)=$ $2 m n-16, f\left(v_{n-6} v_{n-5}\right)=2 m n-12, f\left(v_{n-5} v_{n-4}\right)=2 m n-10, f\left(v_{n-4} v_{n-3}\right)=2 m n-6, f\left(v_{n-3} v_{n-2}\right)=$ $2 m n-8, f\left(v_{n-2} v_{n-1}\right)=2 m n, f\left(v_{n-1} v_{n}\right)=2 m n-2, f\left(v_{n} v_{1}\right)=2 m n-4$.

Label the edges of the circle $C_{n}^{(2)}$ as follows: $f\left(v_{n+i} v_{n+i+1}\right)=4 n(m-1)+2 i, 1 \leq i \leq$ $n-9, f\left(v_{2 n-9} v_{2 n-8}\right)=2 n(2 m-1)-18, f\left(v_{2 n-8} v_{2 n-7}\right)=2 n(2 m-1)-14, f\left(v_{2 n-7} v_{2 n-6}\right)=2 n(2 m-$ 1) $-16, f\left(v_{2 n-6} v_{2 n-5}\right)=2 n(2 m-1)-10, f\left(v_{2 n-5} v_{2 n-4}\right)=2 n(2 m-1)-12, f\left(v_{2 n-4} v_{2 n-3}\right)=2 n(2 m-$ 1) $-6, f\left(v_{2 n-3} v_{2 n-2}\right)=2 n(2 m-1)-8, f\left(v_{2 n-2} v_{2 n-1}\right)=2 n(2 m-1)-4, f\left(v_{2 n-1} v_{2 n}\right)=2 n(2 m-1)-$ $2, f\left(v_{2 n} v_{n+1}\right)=2 n(2 m-1)$.

Label the edges of $C_{n}^{(m)}, C_{n}^{(m-2)}, C_{n}^{(m-4)}, \ldots, C_{n}^{(3)}$ and $C_{n}^{(m-1)}, C_{n}^{(m-3)}, C_{n}^{(m-5)}, \ldots, C_{n}^{(4)}$ as in case (1).

Thus, the labels of corresponding vertices $\bmod (4 m n-2 n)$ will be:

The labels of the vertices of the circle $C_{n}^{(1)}$ are as follows: $f^{*}\left(v_{1}\right) \equiv 0, f^{*}\left(v_{2}\right) \equiv 4 m n-4 n+$ $10, f^{*}\left(v_{3}\right) \equiv 4 m n-4 n+18, f^{*}\left(v_{4}\right) \equiv 4 m n-4 n+22, f^{*}\left(v_{5}\right) \equiv 4 m n-4 n+26, f^{*}\left(v_{6}\right) \equiv 4 m n-4 n+$ $34, f^{*}\left(v_{7}\right) \equiv 4 m n-4 n+42, f^{*}\left(v_{8}\right) \equiv 4 m n-4 n+46, f^{*}\left(v_{9}\right) \equiv 4 m n-4 n+50, f^{*}\left(v_{10}\right) \equiv 4 m n-4 n+$ $58, f^{*}\left(v_{11}\right) \equiv 4 m n-4 n+66, f^{*}\left(v_{12}\right) \equiv 4 m n-4 n+70, f^{*}\left(v_{13}\right) \equiv 4 m n-4 n+74, f^{*}\left(v_{14}\right) \equiv 4 m n-4 n+$ $82, \ldots, f^{*}\left(v_{n-9}\right) \equiv 4 n-56, f^{*}\left(v_{n-8}\right) \equiv 4 n-48, f^{*}\left(v_{n-7}\right) \equiv 4 n-44, f^{*}\left(v_{n-6}\right) \equiv 4 n-40, f^{*}\left(v_{n-5}\right) \equiv$ $4 n-32, f^{*}\left(v_{n-4}\right) \equiv 4 n-24, f^{*}\left(v_{n-3}\right) \equiv 4 n-20, f^{*}\left(v_{n-2}\right) \equiv 4 n-12, f^{*}\left(v_{n-1}\right) \equiv 4 n-4, f^{*}\left(v_{n}\right) \equiv 4 n-6$.

The labels of the vertices of the circle $C_{n}^{(2)}$ are as follows: $f^{*}\left(v_{n+i}\right) \equiv 4 i+2,1 \leq i \leq n-9, f^{*}\left(v_{2 n-8}\right) \equiv$ $4 n-28, f^{*}\left(v_{2 n-7}\right) \equiv 4 n-26, f^{*}\left(v_{2 n-6}\right) \equiv 4 n-22, f^{*}\left(v_{2 n-5}\right) \equiv 4 n-18, f^{*}\left(v_{2 n-4}\right) \equiv 4 n-14, f^{*}\left(v_{2 n-3}\right) \equiv$ $4 n-10, f^{*}\left(v_{2 n-2}\right) \equiv 4 n-8, f^{*}\left(v_{2 n-1}\right) \equiv 4 n-2, f^{*}\left(v_{2 n}\right) \equiv 4 n+2$.

The labels of the vertices of $C_{n}^{(3)}, C_{n}^{(4)}, \ldots, C_{n}^{(m-2)}, C_{n}^{(m-1)}, C_{n}^{(m)}$, respectively, are as in case (1).

Case (5): $n \equiv 9 \bmod 12$.

First, we label the edges of the paths $P_{m}^{(k)}, 1 \leq k \leq n$ beginning with the edges of the path $P_{m}^{(1)}$ the same as in case (1).

Second, we label the edges of the circles $C_{n}^{(k)}, 1 \leq k \leq m$ beginning with the edges of the innermost circle $C_{n}^{(1)}$, then the edges of outer circle $C_{n}^{(m)}$, and then the edges of the circles $C_{n}^{(m-2)}, C_{n}^{(m-4)}, \ldots, C_{n}^{(3)}$.

Finally, we label the edges of the circles $C_{m}^{(m-1)}, C_{m}^{(m-3)}, \ldots, C_{m}^{(2)}$.

Label the edges of the circle $C_{n}^{(1)}$ as follows: $f\left(v_{1} v_{2}\right)=2 n(m-1)+2, f\left(v_{2} v_{3}\right)=2 n(m-1)+$ $4, f\left(v_{3} v_{4}\right)=2 n(m-1)+8, f\left(v_{4} v_{5}\right)=2 n(m-1)+6, f\left(v_{5} v_{6}\right)=2 n(m-1)+10, f\left(v_{6} v_{7}\right)=2 n(m-$ $1)+12, f\left(v_{7} v_{8}\right)=2 n(m-1)+16, f\left(v_{8} v_{9}\right)=2 n(m-1)+14, f\left(v_{9} v_{10}\right)=2 n(m-1)+18, f\left(v_{10} v_{11}\right)=$ $2 n(m-1)+20, f\left(v_{11} v_{12}\right)=2 n(m-1)+24, f\left(v_{12} v_{13}\right)=2 n(m-1)+22, f\left(v_{13} v_{14}\right)=2 n(m-1)+$ $26, \ldots, f\left(v_{n-11} v_{n-10}\right)=2 m n-22, f\left(v_{n-10} v_{n-9}\right)=2 m n-18, f\left(v_{n-9} v_{n-8}\right)=2 m n-20, f\left(v_{n-8} v_{n-7}\right)=$ $2 m n-16, f\left(v_{n-7} v_{n-6}\right)=2 m n-14, f\left(v_{n-6} v_{n-5}\right)=2 m n-10, f\left(v_{n-5} v_{n-4}\right)=2 m n-12, f\left(v_{n-4} v_{n-3}\right)=$ $2 m n-8, f\left(v_{n-3} v_{n-2}\right)=2 m n-6, f\left(v_{n-2} v_{n-1}\right)=2 m n, f\left(v_{n-1} v_{n}\right)=2 m n-2, f\left(v_{n} v_{1}\right)=2 m n-4$.

Label the edges of the circle $C_{n}^{(2)}$ as follows: $f\left(v_{n+i} v_{n+i+1}\right)=4 n(m-1)+2 i, 1 \leq i \leq n-$ $8, f\left(v_{2 n-7} v_{2 n-6}\right)=2 n(2 m-1)-12, f\left(v_{2 n-6} v_{2 n-5}\right)=2 n(2 m-1)-14, f\left(v_{2 n-5} v_{2 n-4}\right)=2 n(2 m-1)-$ $6, f\left(v_{2 n-4} v_{2 n-3}\right)=2 n(2 m-1)-10, f\left(v_{2 n-3} v_{2 n-2}\right)=2 n(2 m-1)-8, f\left(v_{2 n-2} v_{2 n-1}\right)=2 n(2 m-1)-$ $4, f\left(v_{2 n} v_{n+1}\right)=2 n(2 m-1)$. 
Label the edges of $C_{n}^{(m)}, C_{n}^{(m-2)}, C_{n}^{(m-4)}, \ldots, C_{n}^{(3)}$ and $C_{n}^{(m-1)}, C_{n}^{(m-3)}, C_{n}^{(m-5)}, \ldots, C_{n}^{(4)}$ as in case (1). Thus, the labels of corresponding vertices $\bmod (4 m n-2 n)$ will be:

The labels of the vertices of the circle $C_{n}^{(1)}$ are as follows: $f^{*}\left(v_{1}\right) \equiv 0, f^{*}\left(v_{2}\right) \equiv 4 m n-4 n+$ $10, f^{*}\left(v_{3}\right) \equiv 4 m n-4 n+18, f^{*}\left(v_{4}\right) \equiv 4 m n-4 n+22, f^{*}\left(v_{5}\right) \equiv 4 m n-4 n+26, f^{*}\left(v_{6}\right) \equiv 4 m n-4 n+$ $34, f^{*}\left(v_{7}\right) \equiv 4 m n-4 n+42, f^{*}\left(v_{8}\right) \equiv 4 m n-4 n+46, f^{*}\left(v_{9}\right) \equiv 4 m n-4 n+50, f^{*}\left(v_{10}\right) \equiv 4 m n-4 n+$ $58, f^{*}\left(v_{11}\right) \equiv 4 m n-4 n+66, f^{*}\left(v_{12}\right) \equiv 4 m n-4 n+70, f^{*}\left(v_{13}\right) \equiv 4 m n-4 n+74, f^{*}\left(v_{14}\right) \equiv 4 m n-4 n+$ $82, \ldots, f^{*}\left(v_{n-10}\right) \equiv 4 n-60, f^{*}\left(v_{n-9}\right) \equiv 4 n-56, f^{*}\left(v_{n-8}\right) \equiv 4 n-52, f^{*}\left(v_{n-7}\right) \equiv 4 n-44, f^{*}\left(v_{n-6}\right) \equiv$ $4 n-36, f^{*}\left(v_{n-5}\right) \equiv 4 n-32, f^{*}\left(v_{n-4}\right) \equiv 4 n-28, f^{*}\left(v_{n-3}\right) \equiv 4 n-20, f^{*}\left(v_{n-2}\right) \equiv 4 n-10, f^{*}\left(v_{n-1}\right) \equiv 4 n-4$, $f^{*}\left(v_{n}\right) \equiv 4 n-6$.

The labels of the vertices of the circle $C_{n}^{(2)}$ are as follows: $f^{*}\left(v_{n+i}\right) \equiv 4 i+2,1 \leq i \leq n-8, f^{*}\left(v_{2 n-7}\right) \equiv$ $4 n-24, f^{*}\left(v_{2 n-6}\right) \equiv 4 n-22, f^{*}\left(v_{2 n-5}\right) \equiv 4 n-16, f^{*}\left(v_{2 n-4}\right) \equiv 4 n-12, f^{*}\left(v_{2 n-3}\right) \equiv 4 n-14, f^{*}\left(v_{2 n-2}\right) \equiv$ $4 n-8, f^{*}\left(v_{2 n-1}\right) \equiv 4 n-2, f^{*}\left(v_{2 n}\right) \equiv 4 n+2$.

The labels of the vertices of $C_{n}^{(3)}, C_{n}^{(4)}, \ldots, C_{n}^{(m-2)}, C_{n}^{(m-1)}, C_{n}^{(m)}$, respectively, are as in case (1).

Case (6): $n \equiv 11 \bmod 12$.

First, we label the edges of the paths $P_{m}^{(k)}, 1 \leq k \leq n$ beginning with the edges of the path $P_{m}^{(1)}$ the same as in case (1).

Second, we label the edges of the circles $C_{n}^{(k)}, 1 \leq k \leq m$ beginning with the edges of the innermost circle $C_{n}^{(1)}$, then the edges of outer circle $C_{n}^{(m)}$, and then the edges of the circles $C_{n}^{(m-2)}, C_{n}^{(m-4)}, \ldots, C_{n}^{(3)}$.

Finally, we label the edges of the circles $C_{m}^{(m-1)}, C_{m}^{(m-3)}, \ldots, C_{m}^{(2)}$.

Label the edges of the circle $C_{n}^{(1)}$ as follows: $f\left(v_{1} v_{2}\right)=2 n(m-1)+4, f\left(v_{2} v_{3}\right)=2 n(m-1)+$ $2, f\left(v_{3} v_{4}\right)=2 n(m-1)+6, f\left(v_{4} v_{5}\right)=2 n(m-1)+8, f\left(v_{5} v_{6}\right)=2 n(m-1)+12, f\left(v_{6} v_{7}\right)=2 n(m-$ $1)+10, f\left(v_{7} v_{8}\right)=2 n(m-1)+14, f\left(v_{8} v_{9}\right)=2 n(m-1)+16, f\left(v_{9} v_{10}\right)=2 n(m-1)+20, f\left(v_{10} v_{11}\right)=$ $2 n(m-1)+18, f\left(v_{11} v_{12}\right)=2 n(m-1)+22, f\left(v_{12} v_{13}\right)=2 n(m-1)+24, f\left(v_{13} v_{14}\right)=2 n(m-1)+$ $28, \ldots, f\left(v_{n-8} v_{n-7}\right)=2 m n-16, f\left(v_{n-7} v_{n-6}\right)=2 m n-14, f\left(v_{n-6} v_{n-5}\right)=2 m n-10, f\left(v_{n-5} v_{n-4}\right)=$ $2 m n-12, f\left(v_{n-4} v_{n-3}\right)=2 m n-8, f\left(v_{n-3} v_{n-2}\right)=2 m n-6, f\left(v_{n-2} v_{n-1}\right)=2 m n-2, f\left(v_{n-1} v_{n}\right)=2 m n-$ $4, f\left(v_{n} v_{1}\right)=2 m n$.

Label the edges of the circle $C_{n}^{(2)}$ as follows: $f\left(v_{n+i} v_{n+i+1}\right)=4 n(m-1)+2 i, 1 \leq i \leq n-$ $2, f\left(v_{2 n-1} v_{2 n}\right)=4 m n, f\left(v_{2 n} v_{n+1}\right)=4 m n-2$.

Label the edges of $C_{n}^{(m)}, C_{n}^{(m-2)}, C_{n}^{(m-4)}, \ldots, C_{n}^{(3)}$ and $C_{n}^{(m-1)}, C_{n}^{(m-3)}, C_{n}^{(m-5)}, \ldots, C_{n}^{(4)}$ as in case (1).

Thus, the labels of corresponding vertices $\bmod (4 m n-2 n)$ will be:

The labels of the vertices of the circle $C_{n}^{(1)}$ are as follows: $f^{*}\left(v_{1}\right) \equiv 6, f^{*}\left(v_{2}\right) \equiv 4 m n-$ $4 n+10, f^{*}\left(v_{3}\right) \equiv 4 m n-4 n+14, f^{*}\left(v_{4}\right) \equiv 4 m n-4 n+22, f^{*}\left(v_{5}\right) \equiv 4 m n-4 n+30, f^{*}\left(v_{6}\right) \equiv$ $4 m n-4 n+34, f^{*}\left(v_{7}\right) \equiv 4 m n-4 n+38, f^{*}\left(v_{8}\right) \equiv 4 m n-4 n+46, f^{*}\left(v_{9}\right) \equiv 4 m n-4 n+54, f^{*}\left(v_{10}\right) \equiv$ $4 m n-4 n+58, f^{*}\left(v_{11}\right) \equiv 4 m n-4 n+62, f^{*}\left(v_{12}\right) \equiv 4 m n-4 n+70, \ldots, f^{*}\left(v_{n-7}\right) \equiv 4 n-44, f^{*}\left(v_{n-6}\right) \equiv$ $4 n-36, f^{*}\left(v_{n-5}\right) \equiv 4 n-32, f^{*}\left(v_{n-4}\right) \equiv 4 n-28, f^{*}\left(v_{n-3}\right) \equiv 4 n-20, f^{*}\left(v_{n-2}\right) \equiv 4 n-12, f^{*}\left(v_{n-1}\right) \equiv$ $4 n-8, f^{*}\left(v_{n}\right) \equiv 4 n-4$.

The labels of the vertices of the circle $C_{n}^{(2)}$ are as follows: $f^{*}\left(v_{n+1}\right) \equiv 4, f^{*}\left(v_{n+i}\right) \equiv 4 i+2,2 \leq i \leq$ $n-2, f^{*}\left(v_{2 n-1}\right) \equiv 4 n, f^{*}\left(v_{2 n}\right) \equiv 4 n+2$.

The labels of the vertices of $C_{n}^{(3)}, C_{n}^{(4)}, \ldots, C_{n}^{(m-2)}, C_{n}^{(m-1)}, C_{n}^{(m)}$, respectively, are as the same as in case (1).

Illustration: An e.e.g.l. of the cylinder grid graphs $C_{9,3}, C_{7,9}, C_{7,11}, C_{7,13}, C_{7,15}, C_{7,17}$ and $C_{7,19}$ is shown in Figure 14. 


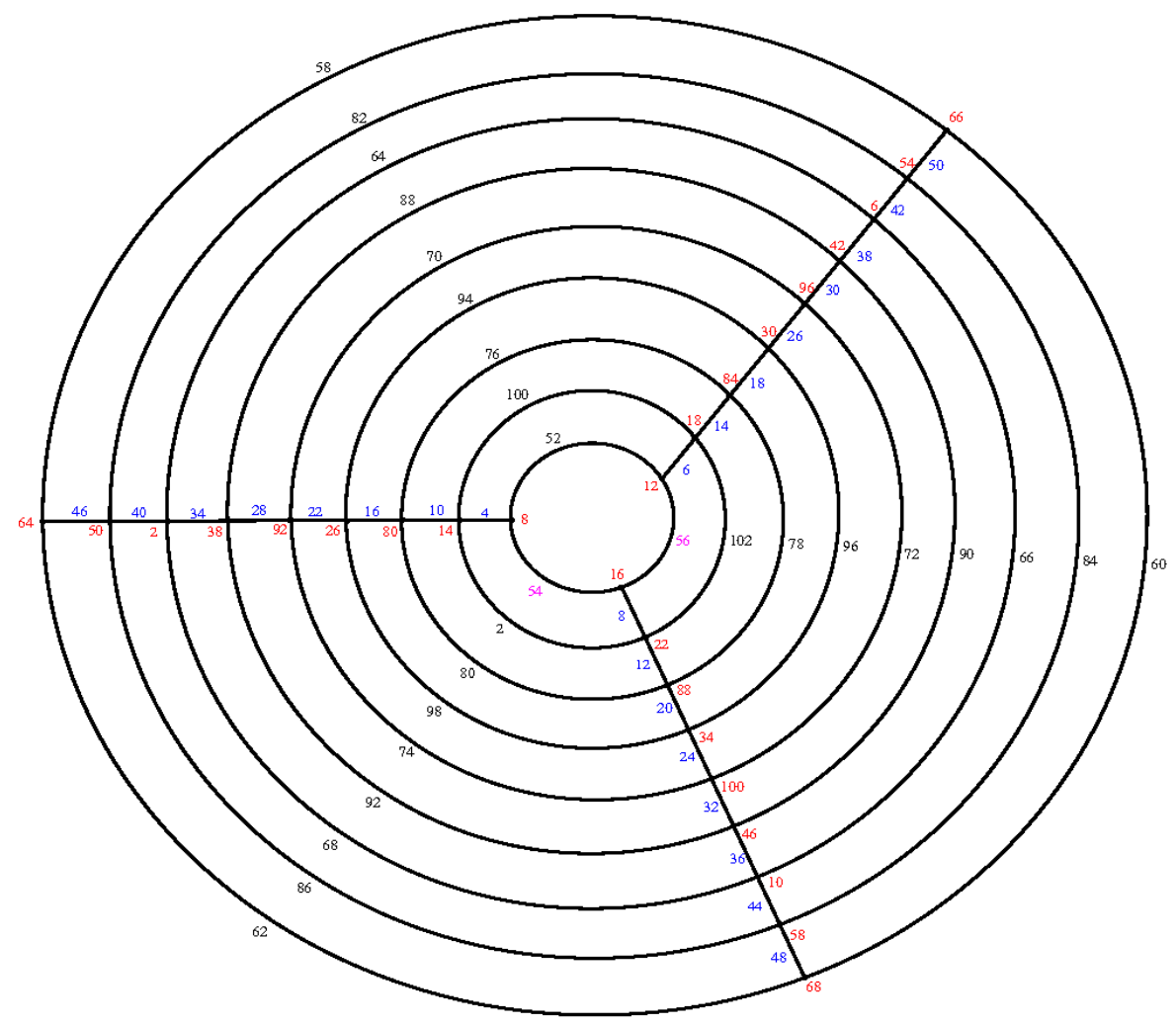

(a) $C_{9,3}$

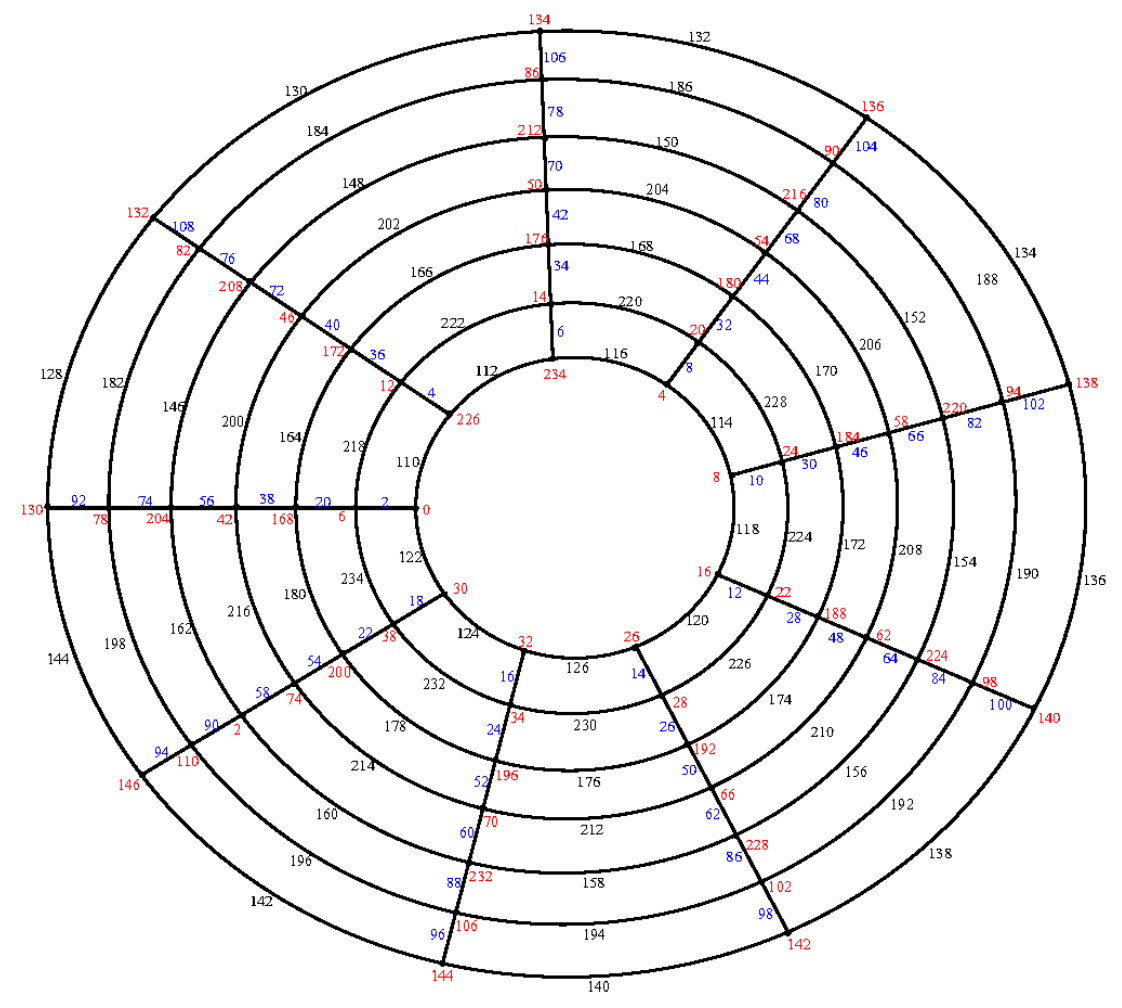

(b) $C_{7,9}$

Figure 14. Cont. 


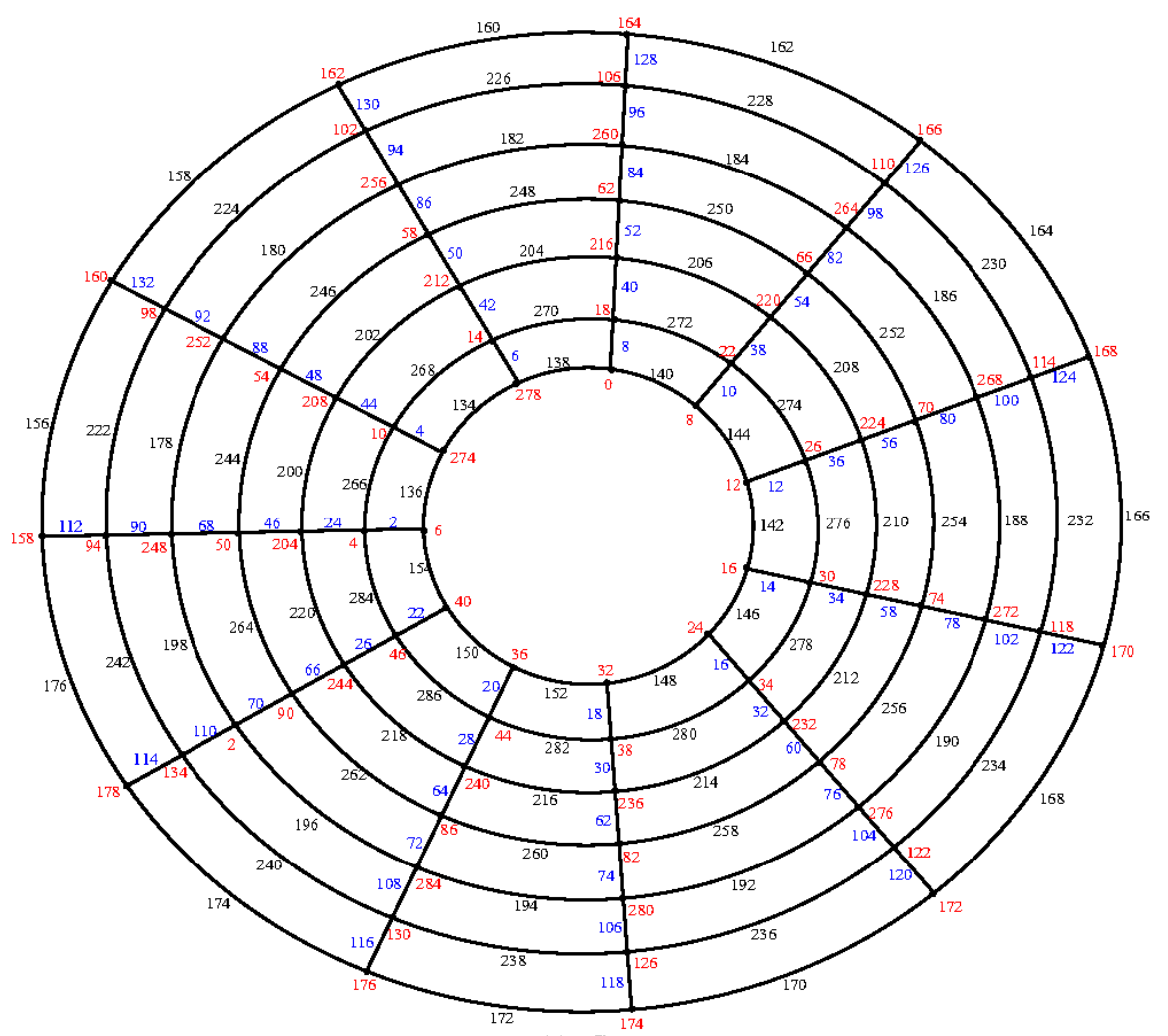

(c) $C_{7,11}$

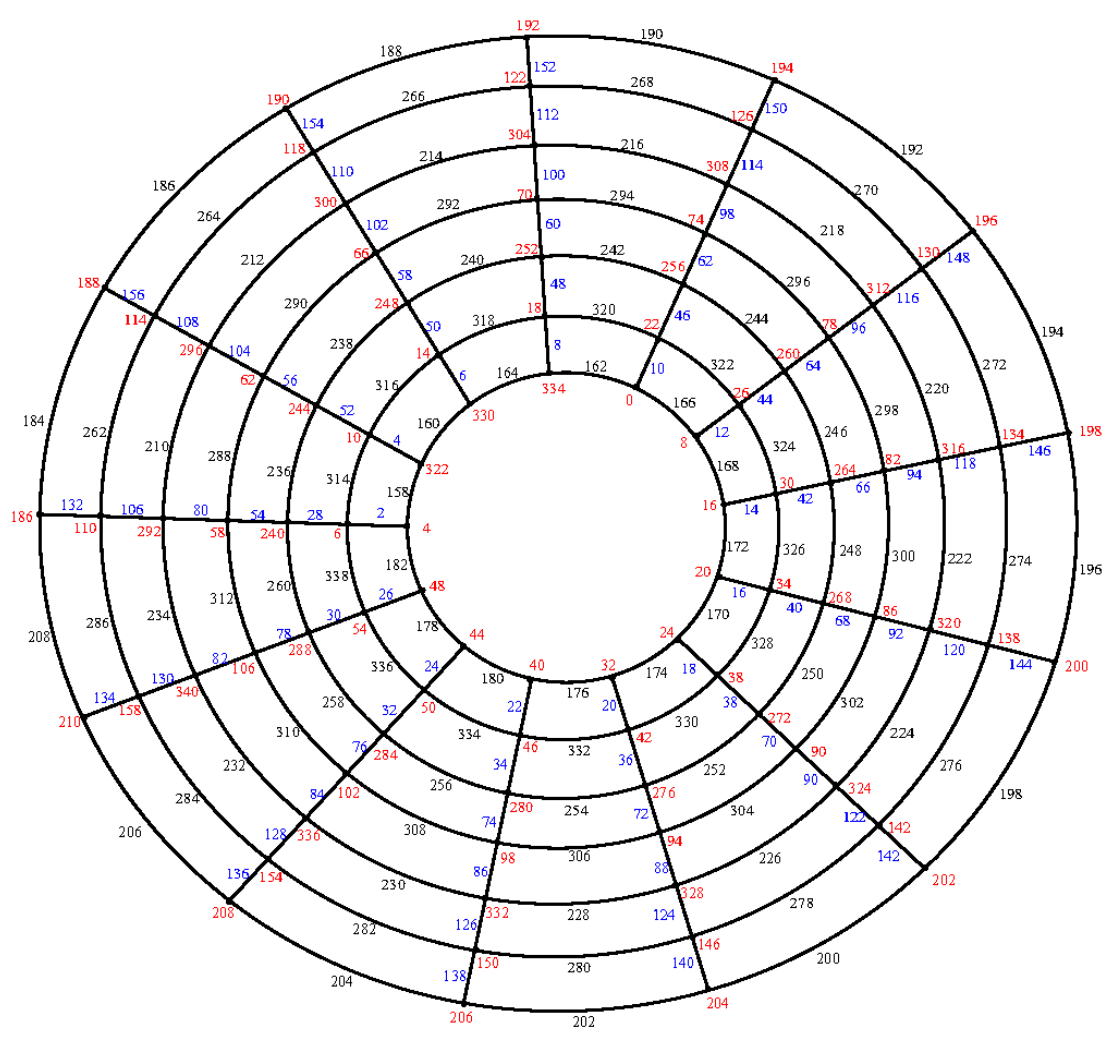

(d) $C_{7,13}$

Figure 14. Cont. 


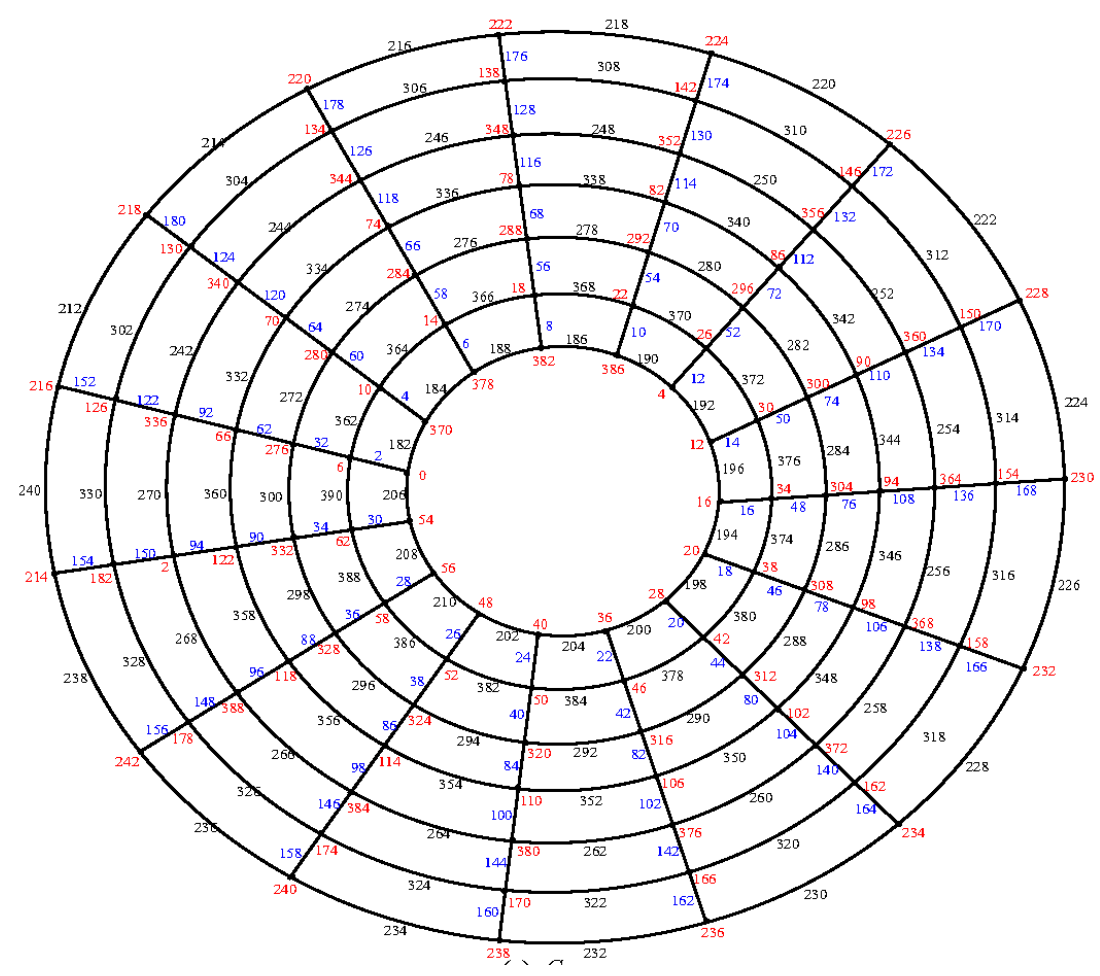

(e) $C_{7,15}$

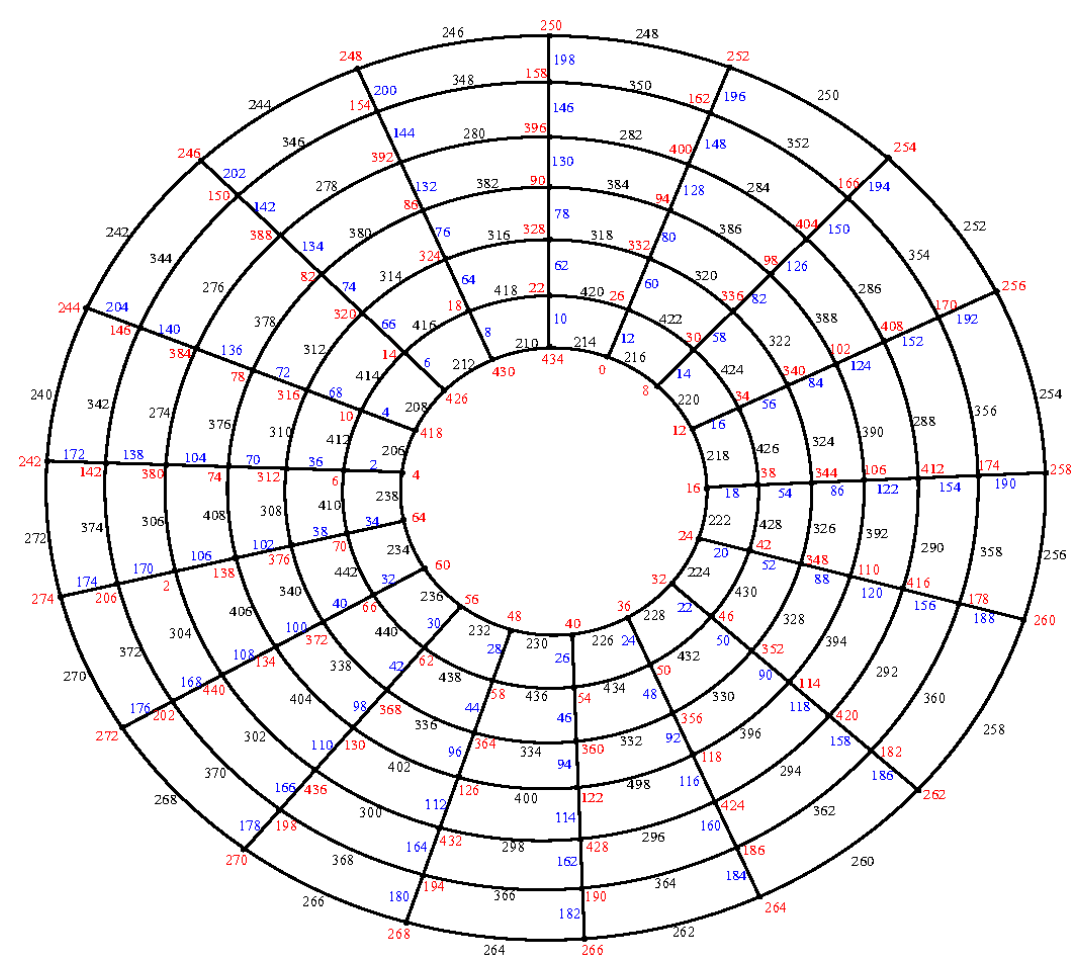

(f) $C_{7,17}$

Figure 14. Cont. 


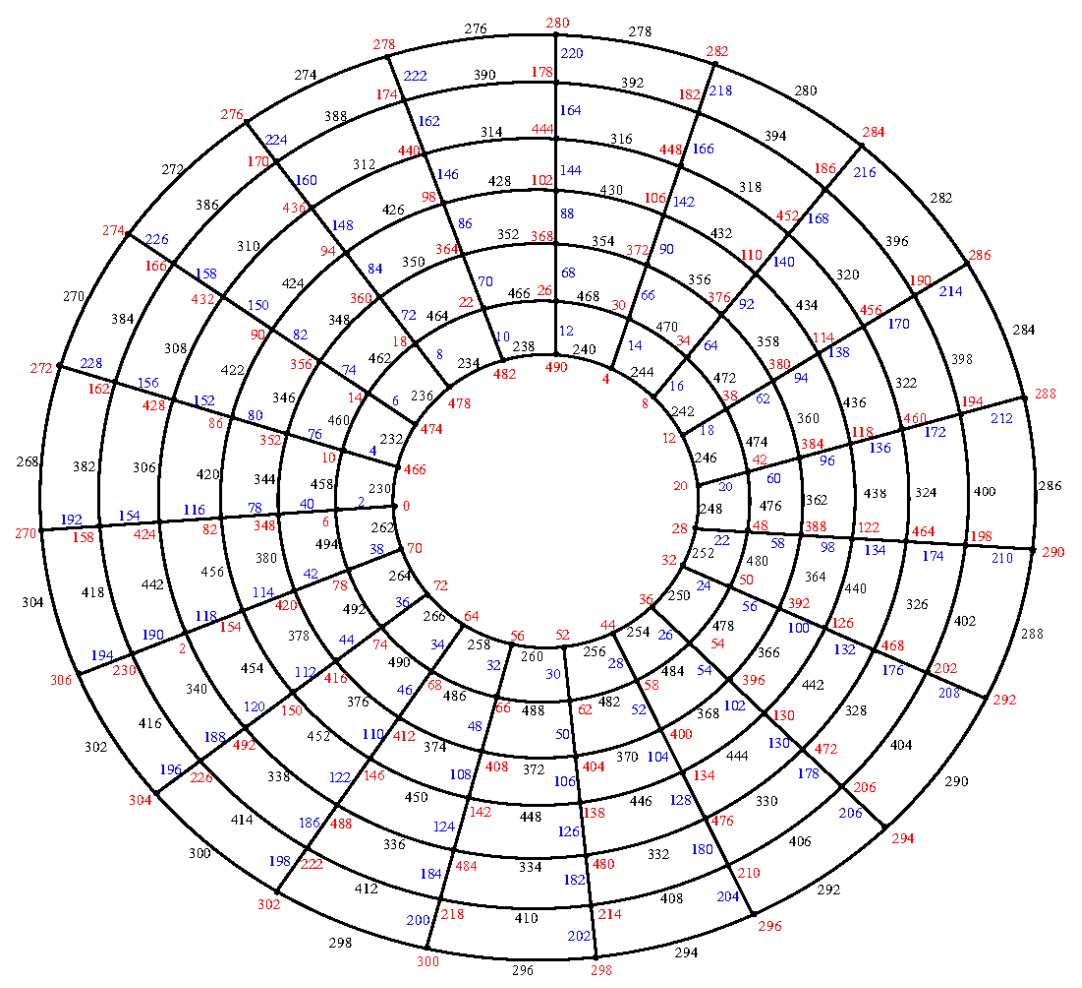

(g) $C_{7,19}$

Figure 14. An e.e.g.l. of the cylinder grid graphs $C_{9,3}, C_{7,9}, C_{7,11}, C_{7,13}, C_{7,15}, C_{7,17}$ and $C_{7,19}$.

\section{Conclusions}

In this paper, using the connection of labeling of graphs with modular arithmetic and theory of numbers in general, we give a detailed study for e.e.g., l. of all cases of members of the cylinder grid graphs. The study of necessary and sufficient conditions for e.e.g., l. of other important families including torus $C_{m} \times C_{n}$ and rectangular $P_{m} \times P_{n}$ grid graphs should be taken into consideration in future studies of e.e.g., 1 .

Author Contributions: All authors contributed equally to this work.

Funding: This work was supported by the deanship of Scientific Research, Taibah University, Al-Madinah Al-Munawwarah, Saudi Arabia.

Acknowledgments: The authors are grateful to the anonymous reviewers for their helpful comments and suggestions for improving the original version of the paper.

Conflicts of Interest: The authors declare that there are no conflicts of interest regarding the publication of this paper.

\section{References}

1. Acharya, B.D.; Arumugam, S.; Rosa, A. Labeling of Discrete Structures and Applications; Narosa Publishing House: New Delhi, India, 2008; pp. 1-14.

2. Bloom, G.S. Numbered Undirected Graphs and Their Uses, a Survey of a Unifying Scientific and Engineering Concept and Its Use in Developing a Theory of Non-Redundant Homometric Sets Relating to Some Ambiguities in X-ray Diffraction Analysis. Ph.D. Thesis, University of Southern California, Los Angeles, CA, USA, 1975.

3. Bloom, G.S.; Golomb, S.W. Numbered complete graphs, unusual rulers, and assorted applications. In Theory and Applications of Graphs, Lecture Notes in Math, 642; Springer: New York, NY, USA, 1978; pp. 53-65.

4. Bloom, G.S.; Golomb, S.W. Applications of numbered undirected graphs. Proc. IEEE 1977, 65, 562-570. [CrossRef] 
5. Bloom, G.S.; Hsu, D.F. On graceful digraphs and a problem in network addressing. Congr. Numer. 1982, 35, 91-103.

6. Graham, R.L.; Pollak, H.O. On the addressing problem for loop switching. Bell Syst. Tech. J. 1971, 50, 2495-2519. [CrossRef]

7. Sutton, M.; Labellings, S.G. Summable Graphs Labellings and Their Applications. Ph.D. Thesis, the University of Newcastle, New South Wales, Australia, 2001.

8. Shang, Y. More on the normalized Laplacian Estrada index. Appl. Anal. Discret. Math. 2014, 8, 346-357. [CrossRef]

9. Shang, Y. Geometric assortative growth model for small-world networks. Sci. World J. 2014, $2014,1-8$. [CrossRef] [PubMed]

10. Shang, Y. Deffuant model of opinion formation in one-dimensional multiplex networks. J. Phys. A Math. Theor. 2015, 48, 395101. [CrossRef]

11. Gross, J.; Yellen, J. Graph Theory and Its Applications; CRC Press: Boca Raton, FL, USA, 1999.

12. Rosa, A. On certain valuations of the vertices of a graph. In Theory of Graphs, Proceedings of the International Symposium, Rome, Italy, July 1966; Gordan and Breach, Dunod: New York, NY, USA, 1967; pp. 349-355.

13. Golomb, S.W. How to Number a Graph. In Graph Theory and Computing; Read, R.C., Ed.; Cademic Press: New York, NY, USA, 1972; pp. 23-37.

14. Gnanajothi, R.B. Topics in Graph Theory. Ph.D. Thesis, Madurai Kamaraj University, Tamil Nadu, India, 1991.

15. Seoud, M.A.; Abdel-Aal, M.E. On odd graceful graphs. Ars Comb. 2013, 108, 161-185.

16. Gao, Z. Odd graceful labelings of some union graphs. J. Nat. Sci. Heilongjiang Univ. 2007, 24, 35-39.

17. Lo, S.P. On edge-gracefullabelings of graphs. Congr. Numer. 1985, 50, 231-241.

18. Kuan, Q.; Lee, S.; Mitchem, J.; Wang, A. On edge-graceful unicyclic graphs. Congr. Numer. 1988, 61, 65-74.

19. Lee, L.; Lee, S.; Murty, G. On edge-graceful labelings of complete graphs: Solutions of Lo's conjecture. Congr. Numer. 1988, 62, 225-233.

20. Solairaju, A.; Chithra, K. Edge-odd graceful graphs. Electron. Notes Discret. Math. 2009, 33, 15-20. [CrossRef]

21. Daoud, S.N. Edge odd graceful labeling of some path and cycle related graphs. AKCE Int. J. Graphs Comb. 2017, 14, 178-203. [CrossRef]

22. Daoud, S.N. Edge odd graceful labeling cylinder grid and torus grid graphs. IEEE Access 2019, 7, 10568-10592. [CrossRef]

23. Daoud, S.N. Vertex odd graceful labeling. Ars Comb. 2019, 142, 65-87.

24. Elsonbaty, A.; Daoud, S.N. Edge even graceful labeling of some path and cycle related graphs. Ars Comb. 2017, 130, 79-96.

25. Daoud, S.N. Edge even graceful labeling polar grid graph. Symmetry 2019, 11, 38. [CrossRef]

26. Gallian, J.A. A dynamic survey of graph labeling. Electron. J. Comb. 2017, 22, \#DS6. 